\title{
The dependence of exhaust power components on edge gradients in JET-C and JET-ILW H-mode plasmas
}

\author{
A R Field ${ }^{1}$, C D Challis ${ }^{1}$, J M Fontdecaba ${ }^{2}$, L Frassinetti ${ }^{3}$, \\ L Horvath ${ }^{1,4}$, Hyun-Tae Kim ${ }^{1}$, C Maggi ${ }^{1}$, C M Roach ${ }^{1}$, \\ S Saarelma ${ }^{1,5}$, M Sertoli $^{6}$ G Szepeisi ${ }^{1}$ and JET contributors* \\ EUROfusion Consortium, JET, Abingdon, Oxon, OX14 3DB, UK. \\ ${ }^{1}$ CCFE, Culham Science Centre, Abingdon, Oxon, OX14 3DB, UK. \\ ${ }^{2}$ Laboratorio Naçional de Fusión Ciemat, 28040 Madrid, Spain. \\ 3 Association VR, Fusion Plasma Physics, KTH, SE-10044, Stockholm, Sweden. \\ ${ }^{4}$ York Plasma Institute, Department of Physics, University of York, York, \\ YO10 5DD, UK. \\ ${ }^{5}$ General Atomics, San Diego, CA 92186-5608, United States of America \\ ${ }^{6}$ Max-Planck-Institut für Plasmaphysik, Boltzmannstrasse 2, D-58748, Garching, \\ Germany. \\ *see the author list of E. Joffrin et al., Nucl. Fusion 59 (2019) 112021 \\ E-mail: anthony.field@ukaea.uk
}

\begin{abstract}
.
Exhaust power components due to ELMs, radiation and heat transport across the edge transport barrier (ETB) between ELMs are quantifed for $\mathrm{H}$-mode plasmas in JET-C and JET-ILW for comparison with simulations of pedestal heat transport. In low-current, JET-ILW pulses with a low rate of gas fuelling, the pedestal heat transport is found not to be stiff, i.e. the effective, mean heat diffusivity $\left\langle\chi_{e f f}\right\rangle$ does not increase with the electron temperature gradient $\left\langle d T_{e} / d R\right\rangle_{\text {ped }}$ across the pedestal and the parameter $\eta_{e}=L_{n_{e}} / L_{T_{e}}$ increases with the conducted loss power across the pedestal, with the latter saturating at mean values $\left\langle\eta_{e}\right\rangle_{\text {ped }} \lesssim 2$. This increase in pedestal temperature gradient is partly due to a relative reduction of the ion neo-classical heat transport (which is more significant at low plasma current) with decreasing collisionality at higher power. In JET-ILW pulses, significantly more power is required at a high gas puffing rate to achieve a similar pedestal pressure and normalised confinement to that in otherwise similar JET-C pulses without gas-puffing. The increased heat transport across the JET-ILW pedestals is caused by changes to the pedestal structure induced by the gas puffing, which is required to mitigate contamination by $\mathrm{W}$ impurities sputtered from the target plates. In high-power JETILW pulses, the radiated power is dominated by that from W, which exhibits a highly asymmetric poloidal distribution due to toroidal rotation. During the ELMy H-mode phase, the $\mathrm{W}$ is concentrated in the outer 'mantle' region $\left(0.7 \leq \rho_{N} \leq 0.96\right)$ inside the pedestal top by a favourable alignment of profile gradients, where it can be effectively flushed by ELMs. Transport analysis reveals that the strong mantle radiation cools the outer region of the plasma, causing more of the heat to be lost through the electron
\end{abstract}


channel. However, direct cooling by $\mathrm{W}$ radiation from the ETB region is shown to be insignificant compared to the power conducted through the pedestal.

PACS numbers: 52.55.-s, 52.65.-y

Submitted to: Plasma Phys. Control. Fusion 


\section{Introduction}

A striking consequence of the change from operation of JET-C with the carbon wall to operation with the ITER-like $(\mathrm{Be} / \mathrm{W})$ wall in JET-ILW is the degradation of confinement due to a reduction in pedestal performance, in particular reduced pedestal temperature [1-4]. Steady type-I ELMy H-mode operation in JET-ILW pulses necessitates gas fuelling to mitigate the influx of tungsten (W) impurities, which are sputtered from the divertor targets. This is in contrast to JET-C, in which gas puffing was not required during the ELMy H-mode phase, partly because outgassing from the graphite wall material provided significant fuelling and also because the carbon (C) impurities contributed significantly $(\sim 40 \%)$ to the total electron density. In JET-C, sputtering of the graphite target material was reduced by efficient cooling the scrape-off-layer (SOL) and divertor plasma by $\mathrm{C}$ radiation, whereas, in JET-ILW, the W impurites radiate mostly from the hotter, confined plasma. Hence, in JET-ILW, significant gas puffing is required to reduce the temperature of the SOL plasma and thereby mitigate the sputtered $\mathrm{W}$ source at the divertor targets. A consquence of this necessity for gas puffing in JET-ILW pulses is to change the structure of the edge transport barrier (ETB) or 'pedestal', in particular by shifting the location of the density pedestal radially outwards relative to that of the temperature. Gas fuelling scans at constant power show that this relative shift increases with the fuelling rate (although there is saturation at higher gas puffing rates), consequently reducing the pressure at the pedestal top and hence the overall confinement [5-7].

The reduction in pedestal temperature (and hence temperature gradient) of the JETILW pedestal compared to that in JET-C at a similar heating power is likely a consequence of increased heat transport across the pedestal. The cause of this has been the focus of numerical modelling efforts, e.g. as reported in Refs. [8-11], and has been attributed to an increase in the turbulent pedestal heat transport due to the altered pedestal structure. In particular, the reduced normalised density gradient $R / L_{n_{e}}\left(\equiv-R\left(d n_{e} / d r\right) / n_{e}\right)$ across JETILW pedestals increases the parameter $\eta=L_{n} / L_{T}$ for both the ions and electrons, which is known to increase the growth rates of temperature gradient driven micro-instabilities (ETG, ITG and MTMs) that cause turbulent transport $[7,11,12]$. One of the aims of this paper is to quantify the inter-ELM loss power through the pedestal for comparison with non-linear, gyrokinetic simulations of the pedestal heat flux, e.g. as reported in Ref. [11].

Before investigating specific cases, in $\S 2.1$ we first explain how the inter-ELM, transport loss power through the pedestal $P_{S e p}^{i E L M}$ can be determined from a power balance calculation for the confined plasma using estimates of the plasma stored energy $W_{p l}$ obtained from fast magnetic equilibrium reconstructions performed using EFIT $++[13,17]$. Although there are issues with the reliability of these estimates for JET-ILW pulses, by means of comparisons with kinetic measurements of ELM energy losses $\Delta W_{E L M}$ presented in $\S 2.2$, we show that the the time-averaged ELM loss power $\left\langle P_{E L M}\right\rangle$ can be determined with sufficient accuracy for our purposes provided the ELM freqency is sufficiently low.

Results of application of this analysis to two particular sets of JET-C and JET-ILW Hmode pulses are presented in $\S 3$. Firstly, in $\S 3.1 .1$ results are presented for a set of low-current $(1.4 \mathrm{MA} / 1.7 \mathrm{~T})$, low average triangularity $(\delta \sim 2.0)$ pulses over which the heating power was varied by a factor $3.6(4.6-16 \mathrm{MW})$ with the lowest gas fuelling rate $\left(3 \times 10^{21} \mathrm{e} / \mathrm{s}\right)$ that still allowed sustained, type-I ELMy H-mode operation with the ILW [18]. At this fuelling rate, the ELM frequency remained low enough $\left(f_{E L M} \lesssim 40 \mathrm{~Hz}\right)$ for our power balance analysis to be reliable. The resulting loss power fractions due to ELMs, radiation and pedestal transport losses are found to be reasonably constant with input power.

Fits to high-resolution Thomson Scattering (TS) measurements of the electron 
temperature and density provide measurements of the H-mode pedestal characteristics. In $\S 3.1 .2$, this data is used to investigate the dependence of the pedestal parameters $\left(T_{e, p e d}\right.$, $n_{e, p e d}$ and $\left.p_{e, p e d}\right)$ and their respective gradients on the transport loss power $P_{S e p}^{i E L M}$ through the pedestal. These results show that, in this series of pulses, the pedestal heat transport can be represented in terms of a mean effective thermal diffusivity $\left\langle\chi_{e f f}\right\rangle$ across the pedestal, which is quite constant as the loss power $P_{S e p}^{i E L M}$ conducted through the pedestal increases with the mean pedestal temperature gradient $\left\langle d T_{e} / d R\right\rangle_{\text {ped }}$. This observation that $\left\langle\chi_{e f f}\right\rangle$ does not increase with $\left\langle d T_{e} / d R\right\rangle_{\text {ped }}$ implies that the pedestal heat transport in these pulses is not particularly stiff.

A more detailed investigation of pedestal heat transport in these low-current pulses is presented in $§ 3.1 .3$, where it is found that the cause of the observed 'non-stiffness' is at least partly due to a decrease in the relative magntitude of the contribution from ion neo-classical heat transport as the pedestal collisionality $\nu_{i, p e d}^{\star}$ decreases with increasing heating power. Also, the normalised, mean electron temperature gradient $\left\langle\eta_{e}\right\rangle_{p e d}=L_{n_{e}} / L_{T_{e}}$, which provides the drive for the turbulent transport, is found to increase with heating power rather than being clamped at a critical value.

Secondly, in the next section $\S 3.2$, we compare the loss powers in two high-power (33 MW), 3.0 MA JET-ILW 'ITER-baseline' $\left(\beta_{N} \sim 2\right)$ scenario pulses with two comparable JET-C pulses with similar normalsied confinement $H_{98, y} \sim 1$ and pedestal pressure $p_{e, p e d}$, which is achieved at much lower $(\sim 60 \%)$ heating power than in the JET-ILW pulses. In spite of the increased power in the JET-ILW pulses, $T_{e, p e d}$ is about half and $n_{e, p e d}$ double that in the JET-C pulses. We also find that the inter-ELM, transport loss power through the JET-ILW pedestal is about twice that in JET-C, in agreement with the most recent simulation results [11], which are summarised in $\S 3.2 .3$.

In $\S 4$, we turn our attention to the radiation losses in these high-power JET-ILW pulses, in which we find that the radiated power fraction $\mathcal{F}_{\text {Rad }} \sim 30-40 \%$ is about double that in the JET-C pulses. The measured distribution of total emissivity is also highly asymmetric, peaking at the low-field side (LFS) of the peripheral 'mantle' region, which is defined here as approximately the outer third of the plasma radius, inside the pedestal top, i.e. $0.7 \leq \rho_{N} \leq \rho_{N, \text { ped }} \sim 0.96$, where $\rho_{N}=\sqrt{\psi_{N}}$ and $\psi_{N}$ is the normalised poloidal flux. In $\S 4.1$, we show this to be consistent with the poloidal redistribution within flux surfaces by toroidal rotation, of tungsten (W) impurities, suputtered from the divertor targets.

In $\S 4.2$, we compare mean, normalised density and temperature gradients $\left(R / L_{n_{e}}\right.$ and $R / L_{T_{e}}$ ) in the pedestal (ETB) and mantle regions for these JET-ILW and JET-C pulses and discuss how these might affect the heat transport in these regions through the parameter $\eta_{e}$, which provides the drive for temperature gradient driven turbulence. Also, we investigate the relative strength of the neo-classical impurity convection, which is proportional to the parameter $\zeta_{N C}=R / 2 L_{T_{i}}-R / L_{n_{i}}$ [19], in these peripheral regions.

In section $\S 4.3$, we avail of an interpretive transport analysis of the high-power, JET-ILW pulse \#92432 to determine the loss powers conducted through the ion and electron channels to the pedestal top. The strong $\mathrm{W}$ radiation from the mantle region cools the electrons, thereby increasing the collisional, ion-electron exchange and hence the fraction of heat transported through the electron channel. This is consistent with strong temperature gradient driven turbulence driven by the high values of $\eta_{e} \sim 5-10$ at the pedestal top [12] and with a high fraction of electron thermal transport through the pedestal [11]. We also estimate that the direct heat loss by radiation from within the ETB represents only a small fraction $(\sim 10 \%)$ of the total power conducted through the pedestal. In $\S 5$ we summarise the main results from each section and, finally, in $\S 6$ we state our overall conclusions and give an outlook towards 
future work.

\section{Determining ELM and inter-ELM loss powers}

Energy is exhausted from the confined plasma by: the time-averaged loss power due to ELMs, i.e. $\left\langle P_{E L M}\right\rangle=\Delta W_{E L M} \times f_{E L M}$ (where $\Delta W_{E L M}$ is the energy loss per ELM and $f_{E L M}$ is the ELM frequency), radiation, neo-classical and turbulent pedestal heat transport during the inter-ELM periods, ionisation and charge-exchange neutral losses. Below, in $\S 2.1$ we describe how the loss power from the confined plasma $P_{S e p}$ can be determined from the time-dependent power balance of the confined plasma. To assess the validity of using magnetic measurements of the stored energy $W_{M H D}$ from EFIT equilibrium reconstructions to estimate the plasma stored energy $W_{p l}$, in $\S 2.2$ we compare average ELM energy losses $\Delta W_{E L M}$ determined from magnetic and kinetic (Thomson scattering) profile measurements. Finally, our calculation of the time-dependent, separatrix loss power $P_{S e p}^{i E L M}(t)$ averaged over many inter-ELM periods is explained in $\S 2.3$.

\subsection{Separatrix loss power from power balance}

In principle, at any instant the loss power from the confined plasma $P_{S e p}$ can be determined from the time-dependent power balance of the confined plasma:

$$
P_{S e p}=P_{a b s}-d W_{p l} / d t-P_{R a d}^{P l}-P_{C X}-P_{0, i o n}
$$

where: $P_{a b s}$ is the absorbed heating power; $d W_{p l} / d t$ is the rate of change of the plasma thermal energy; $P_{R a d}^{P l}$ is the radiation from inside the separatrix; $P_{C X}$ is the power lost by charge-exchange neutrals; and $P_{0, i o n}$ is the power required to ionise the influx of neutral atoms. In the case of JET with NBI and ICRH heating systems: $P_{a b s}=P_{N B}-P_{s h}+P_{R F}+P_{O H}$, where $P_{N B}$ is the injected neutral-beam (NB) power, $P_{s h}$ is the unabsorbed 'shine-through' NB power (calculated using PENCIL [20]), $P_{R F}$ is the absorbed ICRH heating power (assumed here to be equal to the injected ICRH power) and $P_{O H}$ is the Ohmic heating power calculated from equilibrium reconstructions.

As a proxy for the total plasma stored energy $W_{p l}$, we use the MHD stored energy $W_{M H D}=3 / 2 \int\left(p_{\perp}+p_{\|}\right) d V$, where the integral is over the plasma volume $V$. In the case of isotropic pressure $\left(p_{\perp}=p_{\|}\right), W_{M H D}$ is equivalent to $W_{p l}$. For a description of how the GradShafranov equation for the MHD equilibrium [14] can be solved, in the case of a sufficiently elongated, non-circular plasma, to determine the plasma pressure $p(\Psi)$ and current $j_{\phi}(\Psi)$ as functions of the poloidal flux $\Psi$, see Ref. [15-17]. Equilibria are calculated for JET pulses using the EFIT++ code, yielding estimates of $P_{O H}$ and $W_{M H D}$ at 0.4 ms intervals [13].

In a steady state $\mathrm{H}$-mode plasma, the average rate of increase of plasma energy during the inter-ELM periods $d W_{p l} / d t$ must balance the time-averaged, ELM-loss power $\left\langle P_{E L M}\right\rangle$ in order to maintain a constant stored energy $\left\langle W_{p l}\right\rangle$ averaged over many ELM cycles, i.e. over several thermal energy confinement times $\tau_{E, t h} \sim 0.2-0.3 \mathrm{~s}$. The equivalance of $d W_{p l} / d t$ during the inter-ELM periods with $\left\langle P_{E L M}\right\rangle \ddagger$ can be used as an internal consistency check on our analysis.

The power radiated from the confined plasma $P_{R a d}^{P l}$ can be calculated from a weighted sum of horizontally viewing, line-integrated measurements of total intensity measured using

$\ddagger$ In order to calculate $\left\langle P_{E L M}\right\rangle$ from the time series data $\Delta W_{E L M}(t)$, we use the averaging algorithm described in Ref. [22]. 
a multi-chord bolometer system [21]. This system has a sample rate of $5 \mathrm{kHz}$ and uses an adaptive filtering algorithm so as not to smooth fast transients due to ELMs, while having lower bandwidth of typically $0.2 \mathrm{kHz}$ during steady phases. As this calculation of $P_{\text {Rad }}^{P l}$ relies on assumptions on the symmetry of the emissivity on flux surfaces, it tends to overestimate $P_{\text {Rad }}^{P l}$ in the presence of highly in-out asymmetric distributions (as present in high-power JET-ILW plasmas with dominant $\mathrm{W}$ radiation) by as much as $50 \%$. Here, we refer to this fast radiated power signal as $P_{R a d}^{H o r}$.

In order to overcome this problem with the radiation measurements, we use estimates of the $P_{R a d}^{P l}$ from tomographic inversions [23] of the multi-chord bolometer data, which take proper account of the asymmetric emissivity distribution. Reconstructions are calculated at the mid-times of each inter-ELM period, using input data averaged over $5 \mathrm{~ms}$ intervals, and used to form a 'slow' radiated power signal we refer to here as $\left\langle P_{R a d}^{P l}\right\rangle$. We can correct the fast radiated power signal $P_{R a d}^{H o r}$ to provide a more accurate, fast estimate of $P_{R a d}^{P l}$ by matching it to the more accurate, slow signal $\left\langle P_{R a d}^{P l}\right\rangle$ from the tomography using a time-dependent scale factor $\S$. While this procedure may seem complicated, it is necessary if we are to determine the detailed time evolution of $P_{S e p}$ during the inter-ELM periods.

The charge-exchange loss power $P_{C X}$, which is the net energy lost in the form of hot neutral atoms resulting from charge-transfer reactions $\left(D^{+}+D^{0} \rightarrow D^{0}+D^{+}\right)$with the influx of neutral atoms into the confined plasma, can only be obtained from neutral transport calculations based on the measured deuterium influx $\Gamma_{D_{2}}$. The interpretive TRANSP power balance calculations for the JET-ILW pulse \#92432 described in $\S 4.3$ give a mean value of $P_{C X} \sim-0.3 \mathrm{MW}$, i.e. net power deposition just inside the pedestal top. This is caused by a net absorbsion by ionisation of 'warm' CX neutrals from the pedestal region, where the neutral density is higher than further into the confined plasma. The ionisation loss power $P_{0, i o n}$ is also similarly neglibly small in the confined plasma $(\lesssim 0.1 \mathrm{MW})$, although it can be very significant in a strongly recycling divertor plasma. Hence, these components of the total loss power $\left(P_{C X}\right.$ and $\left.P_{0, i o n}\right)$ are not considered further here. Detailed calculations using an edge transport code, including neutral gas dynamics would be required to calculate $P_{C X}$ accurately. Note that for the 1.4 MA pulses discussed in $\S 3.1$, with almost an order of magnitude less gas puffing we would expect $P_{C X}$ to be significantly smaller in magnitude than in the high-power, $3 \mathrm{MA}$ pulse discussed above.

\subsection{Determining ELM energy losses}

For JET-C pulses, which did not require gas puff fuelling during the ELMy H-mode phase, it is possible to determine the ELM energy losses $\Delta W_{E L M}$ from the total stored energy signal $W_{M H D}$ from EFIT equilibrium reconstructions, which decreases promptly at the ELMs, reaching a minimum within $\Delta t_{E L M} \sim 2 \mathrm{~ms}$ of the ELM crash\|. In the case of JET-ILW, however, this method to determine $\Delta W_{E L M}$ from the $W_{M H D}$ signal is less reliable than for JET-C. This is because EFIT uses input signals from magnetic pickup coils located both inside and outside the vessel, the latter being delayed by the presence of the metal wall. Typically, the minimum in the $W_{M H D}$ signal occurs at $\Delta t_{E L M} \sim 10-20 \mathrm{~ms}$ after the ELM crash. Clearly,

$\S$ The signal processing algorithm used to perform this operation is described in $\S$ Appendix A.

$\|$ The ELM crash time is defined here as that of the peak intensity of a visible spectral line $\left(\mathrm{D}_{\alpha}(656 \mathrm{~nm})\right.$ in JET-C and Be II $(527 \mathrm{~nm})$ in JET-ILW) detected by a spectrometer viewing the lower, outer divertor target. This time corresponds to the end of the initial, rapid loss of electron kinetic energy at the initial ELM crash. 

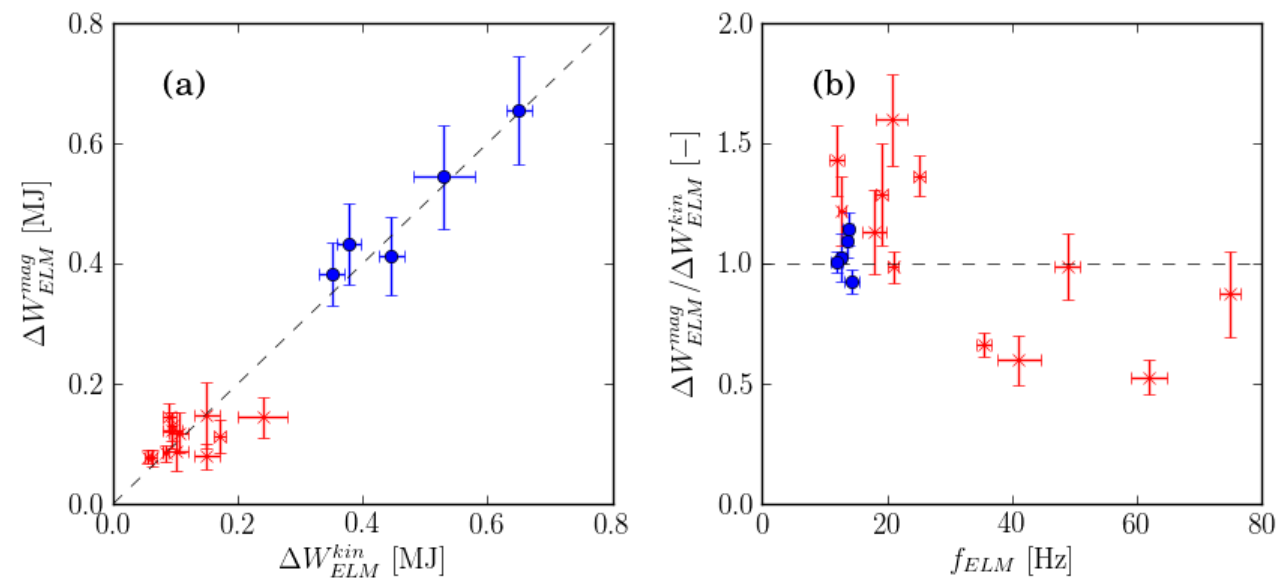

Figure 1: (a) Comparison of kinetic measurements of average ELM energy losses $\triangle W_{E L M}^{k i n}$ with those determined from magnetic measurements $\Delta W_{E L M}^{\text {mag }}$; and (b) the ratio of these losses $\Delta W_{E L M}^{\text {mag }} / \Delta W_{E L M}^{k i n}$ as a function of ELM frequency $f_{E L M}$ for several JET-C $(\bullet)$ and JET-ILW $(\times)$ pulses.

this method can only be used to estimate $\Delta W_{E L M}$ reliably when $f_{E L M} \lesssim 50 \mathrm{~Hz}$ - above this limit $\Delta W_{M H D}$ must underestimate $\Delta W_{E L M}$.

Here, we take the pragmatic approach of determining $\Delta W_{E L M}$ from the drops in the $\triangle W_{M H D}$ signal at the ELMs. Minima and maxima of $W_{M H D}$ within time windows $\left\{t_{E L M}, t_{E L M} \pm 20 \mathrm{~ms}\right\}$ before/after ELM crash times $t_{E L M}$ are used to calculate $\Delta W_{E L M}^{m a g}=$ $\left\{W_{M H D}\right\}_{\max }-\left\{W_{M H D}\right\}_{\text {min }}$. To test the validity of these magenetic ELM energy loss estimates $\Delta W_{E L M}^{m a g}$, they are compared against independent, kinetic estimates of $\Delta W_{E L M}^{k i n}$ obtained from $T_{e}$ and $n_{e}$ profile measurements from the high-resolution Thomson scattering system (HRTS) [24,25], averaged over several ELMs during a pulse.

The kinetic estimates of the ELM losses $\Delta W_{E L M}^{k i n}$ are obtained following the method described in Ref. [26]. Several HRTS $T_{e}$ and $n_{e}$ profle measurements within normalised, preand post-ELM time windows $\left(\Delta \tau_{E L M}=\Delta t_{E L M} / \tau_{E L M}\right.$, where $\Delta t_{E L M}$ is the time relative to the previous ELM and $\tau_{E L M}$ is the inter-ELM time period, of $\sim 80-100 \%$ and $\sim 0-20 \%$ respectively) are binned and fitted separately with modified $\tanh (\operatorname{mtanh}(R)[27])$ functions as a function of major radius $R$. The fitted profiles are then shifted radially to ensure the temperature at the separatrix $T_{e, s e p} \sim 100 \mathrm{eV}$, which is a typical value taken from a two-point SOL model [28]. For further justification for the assumption of a fixed separatrix temperature $T_{e, s e p} \sim 100 \mathrm{eV}$, we refer the reader to Ref. [29]. The shifted profiles are then mapped onto normalised poloidal flux using the mapping $\psi_{N}(R)$ obtained from EFIT equilibria.

By subtracting the volume integrated, post-ELM profiles from the pre-ELM profiles the electron energy loss is then calculated from: $\Delta W_{k i n, e} \approx 3 / 2 \int e\left(\Delta T_{e} n_{e}+\Delta n_{e} T_{e}\right) d V$. Under the assumption that the ion kinetic energy loss $\Delta W_{k i n, i}$ is equal to that lost by the electrons, the total kinetic ELM energy loss is then estimated as $\Delta W_{E L M}^{k i n}=2 \times \Delta W_{k i n, e}$. This latter assumption is equivalent to assuming equal ion and electron pressures $p_{i}=p_{e}$ in the edge region where the ELM energy loss occurs and not as stringent as assuming equal ion and electron temperatures and densities, i.e. that $Z_{\text {eff }}=1$.

In Fig. 1, the averaged ELM losses determined from the magnetic measurements $\Delta W_{E L M}^{m a g}$ are compared with the kinetic measurements $\Delta W_{E L M}^{k i n}$ for a set of JET-C and JET-ILW pulses, including those analysed in $\S 3$ below. To ensure we are comparing like-for-like, for each 
pulse, the $\Delta W_{E L M}^{m a g}$ data is averaged over exactly the same ELMs for which the $\Delta W_{E L M}^{k i n}$ is determined. Whereas, in these JET-C pulses $\Delta W_{E L M}$ was typically $\sim 0.3-0.7 \mathrm{MJ}$, the ELM energy losses in these JET-ILW pulses are much smaller, i.e. $\sim 0.05-0.25 \mathrm{MJ}$. This difference in the typical magnitudes of $\Delta W_{E L M}$ between JET-ILW and JET-C plasmas can be explained by both the higher ELM frequency $f_{E L M}$ and the lower pedestal pressures $p_{e, p e d}$ prevalent in JET-ILW H-mode plasmas.

The ratio of the magnetic to the kinetic estimates of $\Delta W_{E L M}$ are shown in Fig. 1 (b) as a function of ELM frequency. Whereas for the JET-C pulses these estimates agree well (overall average $\Delta W_{E L M}^{\text {mag }} / \Delta W_{E L M}^{\text {kin }} \sim 1.02 \pm 0.07\left(\sigma_{\text {data }}\right)$, the magnetic estimates are much less reliable for the JET-ILW pulses. At lower ELM frequencies $\left(f_{E L M} \leq 35 \mathrm{~Hz}\right)$, the magnetic measurements overestimate the ELM losses by an average factor $\sim 1.2 \pm 0.2\left(\sigma_{\text {data }}\right)$, while at higher ELM frequencies $\left(f_{E L M}>35 \mathrm{~Hz}\right)$ these measurements underestimate $\Delta W_{E L M}$ by an an average factor $\sim 0.65 \pm 0.11\left(\sigma_{\text {data }}\right)$ compared to the kinetic mesurements.

Clearly, when the delay between the ELM crash and the minimum of the $W_{M H D}$ signal is comparable to the inter-ELM period $\tau_{E L M}$, the magnetic mesurements underestimate the ELM losses. As mentioned earlier, the presence of the metallic Be plasma facing components causes additional delays to signals from the magnetic sensors, which limit the time resolution of the magnetic mesurements of the stored energy $W_{M H D}$ for JET-ILW compared to those for JET-C.

It is not clear why $\Delta W_{E L M}^{m a g}$ overestimates $\Delta W_{E L M}^{k i n}$ at low ELM frequency in the JETILW pulses, although the effect of ion dilution by impurities can only partly explain this discrepancy. For the JET-ILW pulses considered here for which $T_{i} \sim T_{e}$ in the edge region and $Z_{\text {eff }} \sim 1.5$ is low, assuming a dominant Be impurity, the reduction in total ion kinetic energy by dilution is $\sim 12 \%$. Hence, assuming equal ion and electron ELM losses overestimates $\Delta W_{E L M}^{\text {kin }}$ by only $\sim 6 \%$, which is insufficient to fully explain the discrepancies shown in Fig. 1 for most pulses. For the JET-C pulses, $Z_{\text {eff }} \sim 2-3$ was typically higher. However, for the pulses considered here, e.g. \#78677 for which kinetic profiles are shown in Fig. 7 , the higher ratio of $T_{i} / T_{e}$ in the edge region results in very similar total pedestal pressures there, which is consistent with the assumption of equal ion and electron ELM energy losses made when calculating $\Delta W_{E L M}^{k i n}$.

Note that the total pressure $p_{t o t}=p_{i}+p_{e}+p_{f}$, where $p_{f}$ is the fast-ion pressure, and hence the magnetic estimates of the stored energy $W_{M H D}$, are not particularly well constrained in MHD equilibrium reconstructions when fitting only to magnetic magnetic measurements. For JET-ILW pulses, such 'standard', magnetics-only EFIT equilibria can overestimate $W_{M H D}$ by up to $\lesssim 20 \%$ compared to results from 'kinetic' EFIT equilibria calculated using the measured kinetic pressure, estimated assuming $p_{k i n}=2 \times p_{e}$, as an additional constraint. However, for JET-C pulses there is often better agreement between these two estimates of $W_{M H D}$. This difference in the degree of agreement is largely explicable by the the higher heating power, and hence higher $p_{f}$, and relatively higher core $p_{i}$, which is partly due to reduced ion dilution by impurities, in JET-ILW pulses compared to those in JET-C pulses (see $\S 4$ ).

We can use these magnetic estimates of the energy ELM losses $\Delta W_{E L M}$ to calculate the time-averaged ELM-loss power $\left\langle P_{E L M}\right\rangle=\left\langle\Delta W_{E L M} \times f_{E L M}\right\rangle \boldsymbol{\uparrow}$. The expected equivalence of $\left\langle P_{E L M}\right\rangle$ and the time-averaged $d W_{p l} / d t$ between the ELMs in a steady-state H-mode phase is used as a check on the consistency of our analysis of the inter-ELM loss power $P_{S e p}^{i E L M}$ in $\S 3.1 .1$ below.

I To perform the running time-averages represented by the $\langle\ldots\rangle$ we used the algorithm described in Appendix B of Ref. [22] 


\subsection{Determination of the inter-ELM loss power}

The time-dependent loss power across the separatrix $P_{S e p}(t)$ can be calculated from the power balance of the confined plasma as expressed by Eq. (1) by using $W_{M H D}$ from the fast-EFIT reconstructions, which is calculated at $0.4 \mathrm{~ms}$ intervals, as a proxy for $W_{p l}$. The $W_{M H D}$ data is first low-pass filtered $\left(f_{L P}=500 \mathrm{~Hz}\right)$ to reduce noise on the $d W_{M H D} / d t$ signal. Also, as there is considerable variation in the detailed time dependence of the inter-ELM quantities, we calculate the time-dependent, inter-ELM loss power $P_{S e p}^{i E L M}(t)$ as an average over many ELM cycles during each period of interest. Also, as the time dependence of the $W_{M H D}$ data is clearly unable to follow the rapid drop in $W_{p l}$ immediately after the ELM crash (which is evident on other diagnostic data, e.g. infra-red (IR) imaging of the target power load, which

has a time resolution of $\sim 0.3 \mathrm{~ms}$ ), we exclude $P_{S e p}^{i E L M}$ data from the first $20 \mathrm{~ms}$ of the ELM cycle in the results presented here.

\section{Results of loss power calculations}

Results are presented here on the loss powers due to ELMs, radiation and the heat transport across the pedestal between ELMs using the core power balance analysis described in $\S 2.1$. Firstly, results are presented in $\S 3.1$ for an input power scan over a series of low plasma current (1.4 MA/1.7 T), type-I ELMy H-mode, JET-ILW pulses with a low rate of gas fuelling. This provides data on how the loss power fractions vary with input power and also on how the inter-ELM pedestal heat transport varies with the pedestal characteristics. Secondly, in $\S 3.2$, we present a comparison of the loss powers in high-power, ITER 'baseline' scenario pulses $\left(\beta_{N} \sim 1.8-2.2\right)$ at 3.0 MA plasma current in JET-C and JET-ILW, for which almost double the heating power was required in JET-ILW to achieve similar pedestal top pressure $p_{e, p e d}$ and H-mode confinement factor $H_{98, y}$ than in the JET-C pulses.

\subsection{4 MA H-mode power scan in JET-ILW with low gas fuelling rate}

3.1.1. Loss-power components Here, we present results of our power balanace analysis for a series of low plasma current (1.4 MA/1.7 T), type-I ELMy H-mode pulses in JET-ILW (\#84791-8), with a low rate of gas fuelling $\left(\Gamma_{D_{2}} \sim 3 \times 10^{21} \mathrm{e} / \mathrm{s}\right)$, over which the input power was increased from $P_{a b s}=4.6$ to $16 \mathrm{MW}[4,18]$. In these experiments the current rise was tailored to achieve a broad central region with $q \gtrsim 1$. Over the range of input power the normalised toroidal- $\beta, \beta_{N} \equiv 100 \beta_{T}\left(B_{\phi} / a I_{M A}\right) \dagger$ increased from $1.5 \rightarrow 3.0$ and, at the highest power, achieved normalised confinement $H_{98, y} \sim 1.2-1.3$, typical of high- $\beta_{N} \sim 2.5-3.0$ 'hybrid' scenario plasmas.

Although the type-I ELM frequency increases with $P_{a b s}$, at the low gas fuelling rate used for these experiments, $f_{E L M}$ remains low enough $(\lesssim 40 \mathrm{~Hz})$ for $\Delta W_{E L M}$ to be determined from the EFIT $W_{M H D}$ signal, albeit with an average systematic discrepancy of a factor $\sim 1.2 \pm 0.2\left(\sigma_{\text {data }}\right)$ with the kinetic estimates $\Delta W_{E L M}^{k i n}$. At higher fuelling rates used in other, related power scans, the ELM frequency is generally higher and this analysis cannot be used

$\dagger$ The toroidal- $\beta$ (normalised pressure) is defined as, $\beta_{T}=\left\langle p_{p l}\right\rangle /\left(B_{t, 0}^{2} / 2 \mu_{0}\right)$, where $\left\langle p_{p l}\right\rangle$ is the volumeaveraged plasma pressure, $B_{t, 0}$ is the toroidal field at the geometrical major radius $R_{0}$ and $I_{M A}$ is the plasma current in MA. Here, the volume-averaged plasma pressure $\left\langle p_{p l}\right\rangle$ may or may not include the fast-ion pressure. The values of $\beta_{N}$ quoted here for 'hybrid' scanario plasmas are calculated using the total plasma pressure, including the fast-ion component. 

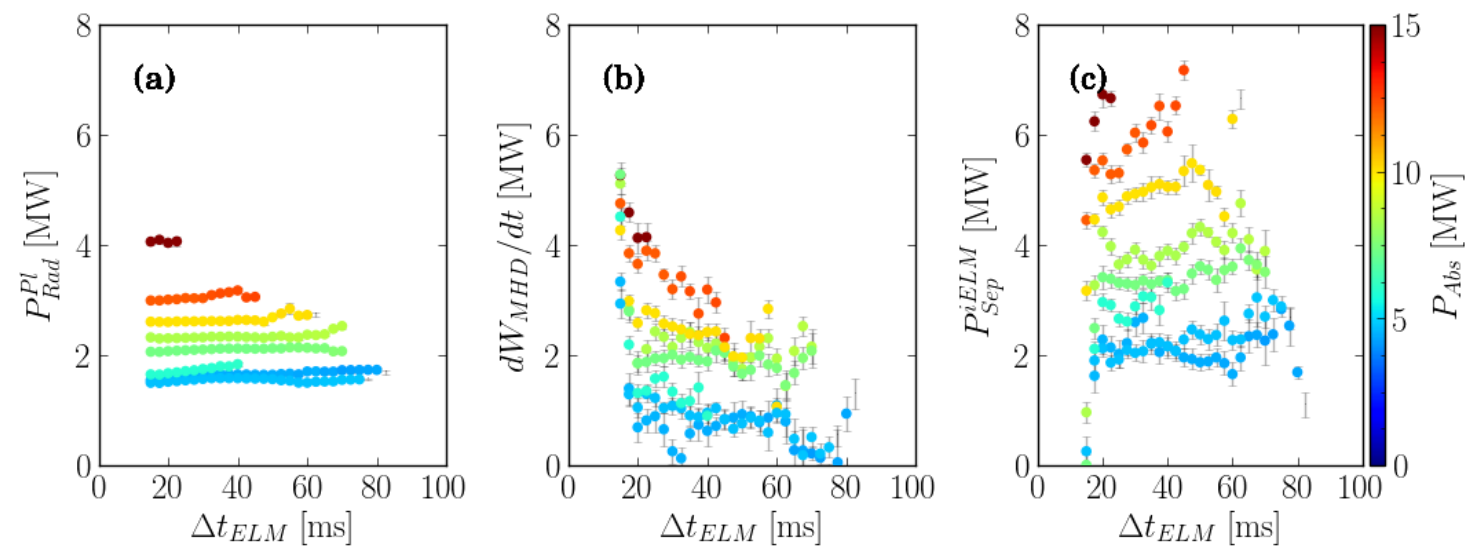

Figure 2: Components of the inter-ELM power balance for the 1.4 MA/1.7 T, low-gas JET-ILW pulses \#84971-8, showing: (a) radiated power $P_{R a d}^{P l}$; (b) rate of change of stored energy $d W_{M H D} / d t$; and (c) the separatrix loss power $P_{S e p}^{i E L M}$ as a function of time from the previous ELM, $\Delta t_{E L M}$, where the colors represent $P_{a b s}$.

reliably. $\ddagger$

Components of the inter-ELM power balance for this series of pulses are shown in Fig. 2. During the intial few ms after the ELM crash (not shown in Fig. 2 (a)), the radiated power increases strongly, particularly from the divertor and SOL, however, this returns to a more steady level after $\Delta t_{E L M} \gtrsim 10 \mathrm{~ms}$. During the inter-ELM period, the rate of increase of plasma stored energy $d W_{M H D} / d t$ (Fig. $2(\mathrm{~b})$ ) decreases modestly with $\Delta t_{E L M}$ because both the radiated power $P_{R a d}^{P l}$ and the pedestal heat transport $P_{S e p}^{i E L M}$ increase due to the increasing density and temperature at the pedestal top.

As the input power $P_{a b s}$ is increased from 4.6 to $16 \mathrm{MW}$, the ELM frequency $f_{E L M}$ (shown in Fig. 3 (a)) increases from $\sim 12$ to $40 \mathrm{~Hz}$, while the fractional loss powers $\mathcal{F}_{X}=P_{X} / P_{a b s}$ in all three loss channels (shown in Fig. 3 (b)) remain approximately constant. Both radiation and ELMs account for similar fractions, i.e. $\mathcal{F}_{\text {Rad }} \sim \mathcal{F}_{E L M} \sim 20-35 \%$, , while the fraction due to inter-ELM conduction loss across the pedestal, $\mathcal{F}_{S e p} \sim 40-50 \%$, is considerably higher. As a consistency check on our analysis, the fraction of power $\mathcal{F}_{d W / d t}$ represented by the rate of increase of plasma stored energy between the ELMs $d W_{M H D} / d t$, has also been shown to be of similar magnitude $( \pm 20 \%)$ to the average ELM loss power $\left\langle P_{E L M}\right\rangle$, as must be the case in a steady-state, ELMy H-mode phase. The residual loss power across the separatrix $P_{S e p}^{i E L M}$ represents $\sim 40-50 \%$ of the input power.

Although results at higher fuelling rates, for which the pedestal performance is further degraded, would be of considerable interest, the resulting ELM frequency is too high for the reliable application of this technique.

3.1.2. Dependence of inter-ELM loss power on pedestal gradients Both turbulent and neo-classical, collisional transport are driven by gradients of the temperature and density. In the case of turbulent transport, various micro-instabilities might explain the heat transport through the ETB. These are driven by gradients in the pressure, temperature or density, e.g. kinetic ballooning modes (KBMs) [27] are driven by the pressure gradient $d p_{e} / d R$, while microtearing modes (MTM) [12] or ion/electron temperature gradient (ITG/ETG) instabilities $\ddagger$ These power scans used 'medium' and 'high' fuelling rates of $\Gamma_{D_{2}} \sim 8 \times 10^{21} \mathrm{e} / \mathrm{s}$ and $1.8 \times 10^{22} \mathrm{e} / \mathrm{s}[4,5]$. 

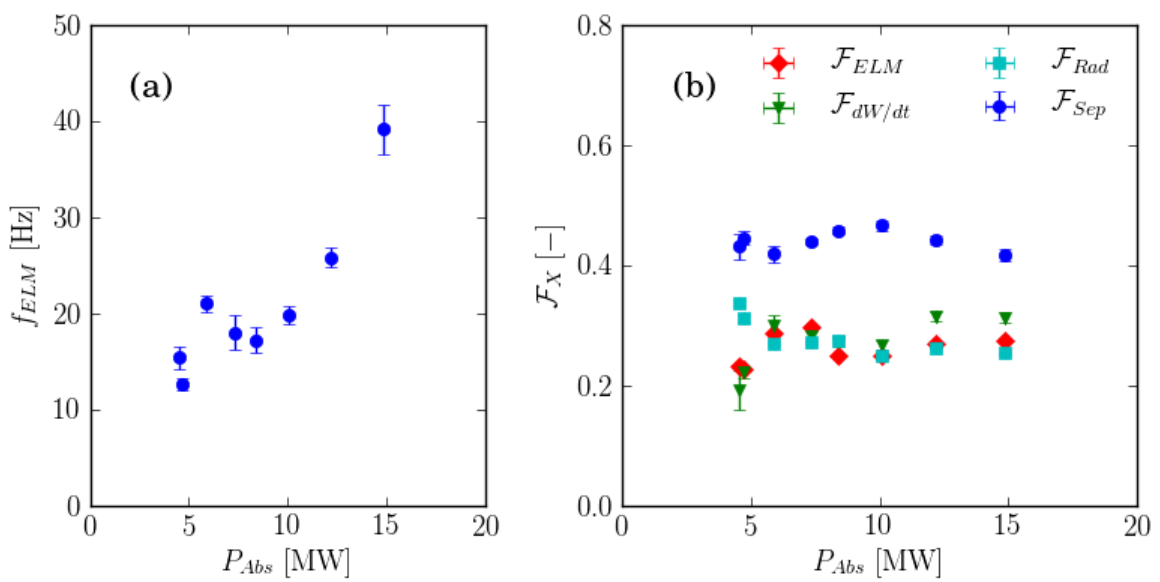

Figure 3: (a) The average ELM frequency $f_{E L M}$ and (b) the time-averaged fractions of loss power $\mathcal{F}_{X}=P_{X} / P_{a b s}$ represented by: ELM losses $\mathcal{F}_{E L M}(\downarrow)$ and the change in plasma energy $\mathcal{F}_{d W / d t}(\nabla)$; radiated power $\mathcal{F}_{\text {Rad }}(\square)$; and the resulting separatrix loss power $P_{\text {Sep }}^{i E L M}(\bullet)$ as a function of the absorbed power $P_{a b s}$ for the same series of pulses shown in Fig. 2.

are driven by a high value of the temperature gradient relative to that of the density, $\eta_{i, e}=L_{n_{e}} / L_{T_{i, e}}$ and trapped-electron modes (TEM) also by the density gradient [30,31]. $\S$. Hence, we investigate below the dependence of the H-mode pedestal (ETB) gradients on the inter-ELM loss power $P_{S e p}^{i E L M}$ in order to determine which parameter exhibits the strongest correlation.

The pedestal profiles are parameterised by fitting the HRTS data $\left(T_{e}, n_{e}\right.$ and $p_{e}$ as a function of major radius $R$ over the edge region $\left.\rho_{N} \gtrsim 0.7\right)$ with $\operatorname{mtanh}(R)$ functions [27]. This provides metrics describing the respective values at the pedestal top, their gradients in the mantle and across the ETB and the position and width of the ETB and a constant offset in the SOL. Here, we calculate the mean gradient of parameter $X$ in the ETB using: $\langle d X / d R\rangle_{\text {ped }}=\left(X_{\text {ped }}-X_{\text {offs }}\right) / \Delta R_{\text {ped }}$, where $X_{\text {ped }}$ and $X_{\text {offs }}$ are the values at the pedestal top and the SOL offset and $\Delta R_{\text {ped }}$ is the pedstal width. Note that the fitting procedure takes into account the finite spatial resolution of the HRTS system $\Delta \sim 1-2 \mathrm{~cm}$ [25]. By subtracting the time of the previous ELM from that of the measurements at each of the TS laser pulses $(50 \mathrm{~Hz}$ repetition rate), these parameters are thereby available as a function of the relative inter-ELM time $\Delta t_{E L M}$.

The evolution of the pedestal top parameters $\left(T_{e, p e d}, n_{e, p e d}\right.$ and $\left.p_{e, p e d}\right)$ and their mean radial gradients across the pedestal are shown in Fig. 4 as a function of $\Delta t_{E L M}$. As these plots show data for all pulses from the 1.4 MA power scan, the color of the data points is used to represent the thermal loss power $P_{l, t h}$, i.e. the net loss power across the separatrix, including radiation $\|$. At any particular time $\Delta t_{E L M}$ after the ELM crash, as $P_{l, t h}$ increases $(4.6 \rightarrow 16 \mathrm{MW}): T_{e, p e d}$ approximately doubles, while $n_{e, p e d}$ decreases by $\sim 25 \%$, with concomitant changes to the respective radial gradients. These trends are broadly consistent with results reported in Refs. [4,5].

$\S$ The gradient scale length $L_{X}$ of parameter $X$ is defined as $1 / L_{X}=-(d X / d R) / X$, i.e. postive for a normal, peaked profile.

$\|$ The thermal loss power, defined as $P_{l, t h}=P_{a b s}-\left\langle d W_{p l} / d t\right\rangle$, which in a steady-state phase is equivalent to $P_{a b s}$. The parentheses $\langle\ldots\rangle$ represent a time-average over many ELM cycles. 

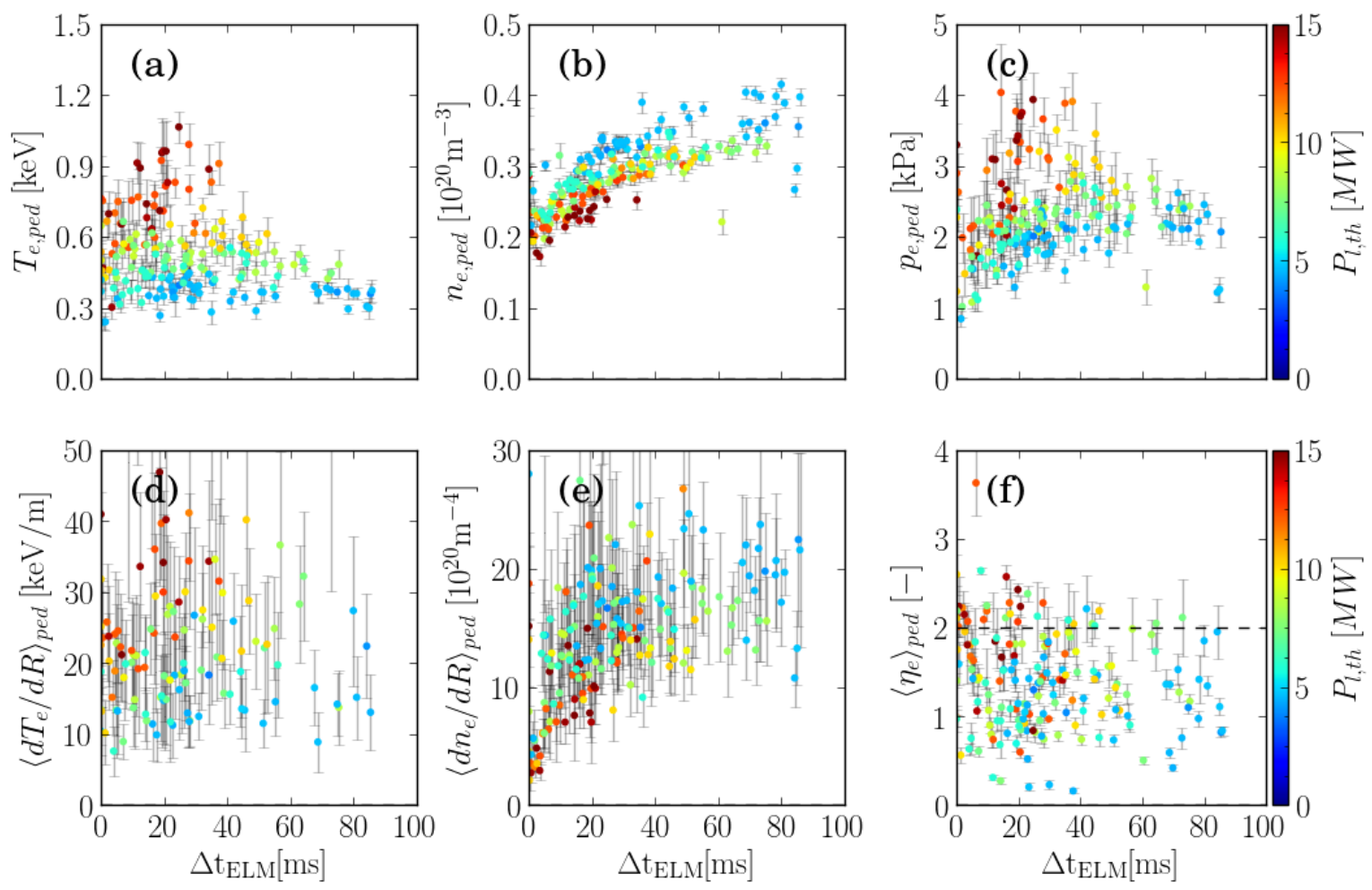

Figure 4: Plasma parameter values at the pedestal top for the same JET-ILW pulses shown in Fig. 3: (a) the electron temperature $T_{e, p e d}$; (b) density $n_{e, p e d}$ and (c) pressure $p_{e, p e d}$; the mean radial gradients (d) $\left\langle d T_{e} / d R\right\rangle_{\text {ped }}$ and (e) $\left\langle d n_{e} / d R\right\rangle_{\text {ped }}$ and (f) the mean parameter $\left\langle\eta_{e}\right\rangle_{\text {ped }}=L_{n_{e}} / L_{T_{e}}$ as a function of the time from the previous $E L M, \Delta \tau_{E L M}$, where $\langle\ldots\rangle_{\text {ped }}$ represents an average over the density pedestal (ETB) region and the color represents the thermal loss power $P_{l, t h}$ for the same series of pulses shown in Fig. 2.

Note that the rate at which $T_{e, p e d}$ and $p_{e, p e d}$ recover after the ELM crash increases with the loss power $P_{l, t h}$, while the opposite is the case for $n_{e, p e d}$, which increases slightly more slowly at higher power. The pre-ELM value of $n_{e, p e d}$ increases with the inter-ELM period $\tau_{E L M}=1 / f_{E L M}$, which is longer at lower power. Also, the pre-ELM value of $p_{e, p e d}$ increases with input power, i.e. at higher power, ELMs are triggered at higher $p_{e, p e d}$ after a shorter inter-ELM period (and vice versa at lower power).

The normalised parameter $\eta_{e}=L_{n_{e}} / L_{T_{e}}$ (and the equivalent parameter $\eta_{i}=L_{n_{e}} / L_{T_{i}}$ ) are the drives for temperature gradient driven instabilities. In Fig. 4 (f), mean values $\left\langle\eta_{e}\right\rangle_{\text {ped }}$ over the full width of the density pedestal are shown as a function of the time after the ELM crash $\Delta t_{E L M}$. These mean gradients are averages over the region $R_{\text {ped }} \pm \Delta R_{\text {ped }} / 2$, where $R_{\text {ped }}$ and $\Delta R_{\text {ped }}$ are the density pedestal position and width parameters from the mtanh() fits. The errorbars, representing $\left\langle\eta_{e}\right\rangle_{\text {ped }} \pm \sigma_{\text {data }}$, indicate the considerable variation of $\eta_{e}$ over the ETB region. At the highest loss power $P_{l, t h},\left\langle\eta_{e}\right\rangle_{p e d}$ saturates early in the ELM cycle at $\sim 2$, consistent with the values quoted in [5], which is a typical threshold value for triggering

I Note that the pre-ELM values of the quantities plotted in Fig. 4 are given by the latest data point, i.e. that at the largest $\Delta t_{E L M}$, at a particular level of inter-ELM power $P_{S e p}^{i E L M}$ (color). 

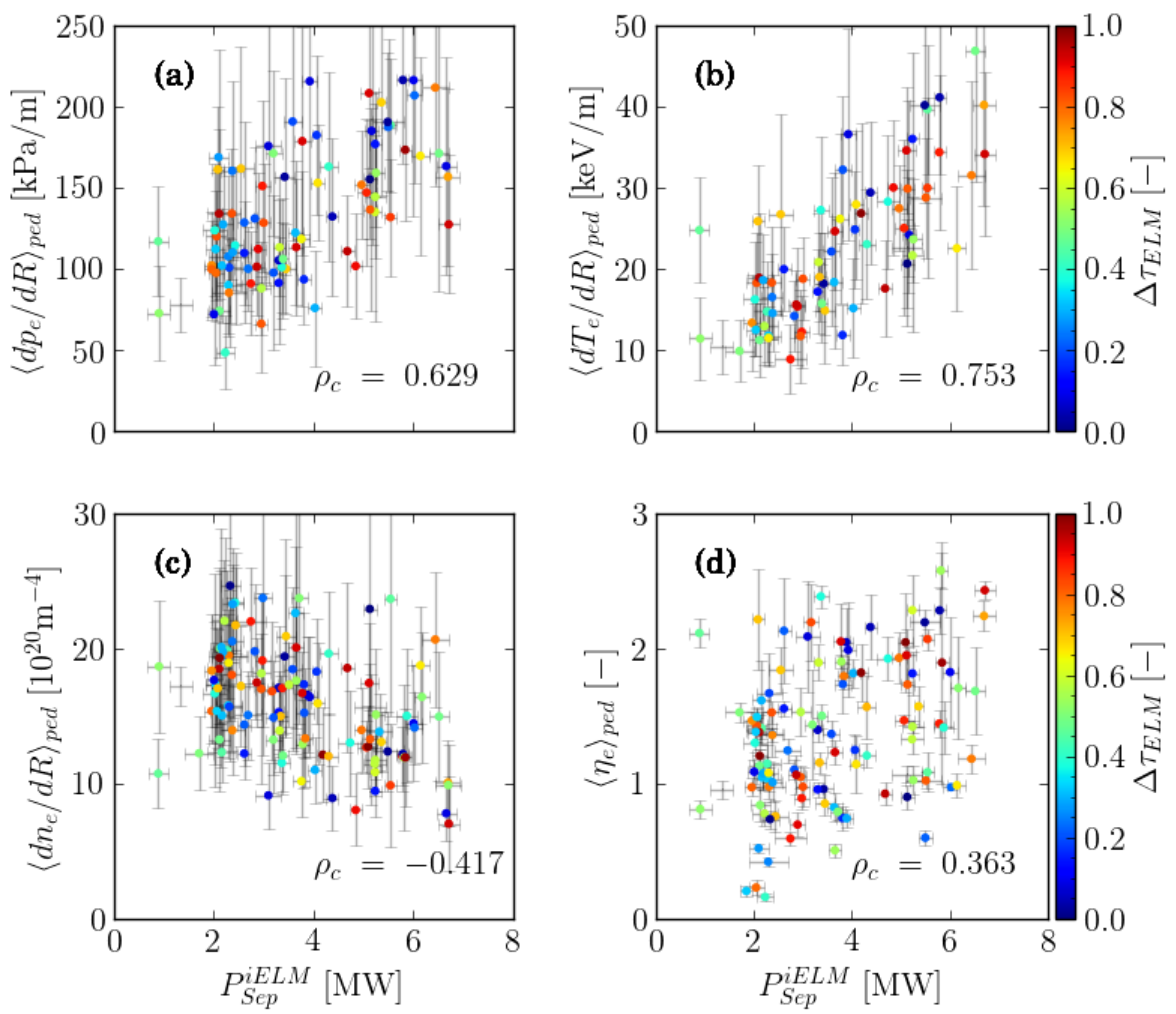

Figure 5: The dependence of the mean values of the electron: (a) pressure gradient $\left\langle d p_{e} / d R\right\rangle_{\text {ped }}$; (b) temperature gradient $\left\langle d T_{e} / d R\right\rangle_{\text {ped }}$; (c) density gradient $\left\langle d n_{e} / d R\right\rangle_{\text {ped }}$; and (d) the mean parameter $\left\langle\eta_{e}\right\rangle_{\text {ped }}$ across the ETB on the inter-ELM loss power PSep $P_{\text {Se }}^{i E L}$ for the pulses shown in Fig. 2, where the color represents the normalised time during the ELM cycle $\Delta \tau_{E L M}$.

electron-temperature-gradient (ETG) driven instabilities, while at lower power somewhat lower values of $\left\langle\eta_{e}\right\rangle_{\text {ped }} \sim 1-2$ prevail, although the scatter of data is large. Micro-instabilities are expected to be driven most strongly where $\eta_{e}$ is highest, which is typically at the top of the pedestal $[7,12]$.

The pedestal gradients $\left\langle d T_{e} / d R\right\rangle_{p e d},\left\langle d n_{e} / d R\right\rangle_{p e d}$ and $\left\langle d p_{e} / d R\right\rangle_{p e d}$ and also the parameter $\eta_{e}$ averaged over the width of the ETB region are shown in Fig. 5 as a function of the inter-ELM loss power $P_{S e p}^{i E L M}$. In each case, the Pearson correlation coefficient $\rho_{c}{ }^{+}$of the respective parameter with $P_{S e p}^{i E L M}$ is shown. The strongest positive correlation (0.75) is with the temperature gradient $\left\langle d T_{e} / d R\right\rangle_{\text {ped }}$, with a somewhat weaker correlation $(0.63)$ with $\left\langle d p_{e} / d R\right\rangle_{\text {ped }}$. The negative correlation (-0.42) of the density gradient $\left\langle d n_{e} / d R\right\rangle_{\text {ped }}$ with $P_{\text {Sep }}^{i E L M}$ perhaps implies that some instability limits the pressure gradient, e.g. KBMs [27]. If this were the case, the increasing temperature gradient with heating power would have to be compensated by a reduction of the density gradient. The location of the particle source might also move outwards as $T_{e, p e d}$ increases with power due to a reduction in penetration of the neutrals.

Although it is evident from Fig. $4(\mathrm{~d}-\mathrm{e})$ that the pedestal gradients increase during the

+ The Pearson correlation coefficient is defined as $\rho_{x y}=C_{x y} /\left(\sigma_{x} \sigma_{y}\right)$, where $C_{x y}$ is covariance of $x$ and $y$ and $\sigma_{x, y}$ are the standard deviations of $x$ and $y$ respectively [32]. 
inter-ELM period, the data shown in Fig. 5 shows little correlation with the normalised, interELM time $\Delta \tau_{E L M}=\Delta t_{E L M} / \tau_{E L M}$ (color). This is because most recovery of the pedestal gradients occurs during the first $20 \mathrm{~ms}$ of the ELM cycle from which times the unreliable $P_{\text {Sep }}^{i E L M}$ data has been excluded.

The approximately linear dependence of $\left\langle d T_{e} / d R\right\rangle_{\text {ped }}$ on $P_{S e p}^{i E L M}$ implies a flux-gradient relation of the form $q_{t o t}=-e n_{e}\left\langle\chi_{e f f}\right\rangle\left\langle d T_{e} / d R\right\rangle_{\text {ped }}$, where $\left\langle\chi_{e f f}\right\rangle$ is an average, effective thermal diffusivity across the pedestal. Here, we make the implicit assumption that a constant fraction of the total heat flux is carried by electron heat conduction. Assuming $q_{t o t}=P_{\text {Sep }}^{i E L M} / S$, where $S \sim 140 \mathrm{~m}^{2}$ is the plasma surface area for these pulses and a mean density in the ETB of $n_{e, p e d} / 2 \sim 1.5 \times 10^{19} \mathrm{~m}^{-3}$, this relation yields a mean value of $\left\langle\chi_{\text {eff }}\right\rangle \sim 0.6 \mathrm{~m}^{2} / \mathrm{s}$ over the power scan. This linear dependence over the power scan implies that, at least in an operational sense, the pedestal heat transport is not particularly 'stiff', i.e. $\left\langle\chi_{e f f}\right\rangle$ does not increase strongly with the temperature gradient. The observed scaling of the pedestal heat transport in this series of pulses is investigated in detail in the next section $\S 3.1 .3$.

\subsubsection{Investigation of pedestal heat transport 'stiffness' In this section we investigate} in more detail the dependence of the pedestal heat transport on heating power in these lowcurrent (1.4 MA/1.7 T) JET-ILW pulses. In particular, how the ratio of the total pedestal heat flux to the neo-classical component $q_{t o t} / q_{i, N C}$ varies with the heating power, and hence the pedestal collisionality $\nu_{i, p e d}^{\star}$; and also the dependence of the total, inter-ELM loss power $P_{S e p}^{i E L M}$ on the mean normalised electron temperature gradient $\left\langle\eta_{e}\right\rangle_{\text {ped }}$, which is the drive for the turbulent component of the heat flux carried by the electrons across the pedestal. Note that in the analysis presented here, we have made use of profile measurements of $T_{e}$ and $n_{e}$ using the high-resolution TS system and have not made use of measurements of $T_{i}$ that are available on JET-ILW from the edge charge-exchange recombination spectroscopy (CXRS) system [40]. As is discussed in more detail below, to obtain an estimate of neo-classical, ion heat flux across the pedestal $q_{i, N C}$, we make the assumption that the average ion temperature gradient $\left\langle d T_{i} / d R\right\rangle_{p e d}$ is a fixed fraction of $\left\langle d T_{e} / d R\right\rangle_{p e d}$ over the density pedestal region.

The collisional, neo-classical component of the pedestal heat flux is given by $q_{i, N C}=$ $-n_{i} \chi_{i, N C}\left\langle d T_{i} / d R\right\rangle_{p e d}$, where the diffusivity scales as $\chi_{i, N C} \propto q^{2} \rho_{i}^{2} / \tau_{i i} \propto q^{2} n_{i} / \sqrt{T_{i}}$, with an enhancement by the factor $\epsilon^{-3 / 2}$ in the low-collisionality, 'banana' regime (see $\S 4.6$ of Ref. [33]). Here, the safety factor $q=d \Phi / d \Psi$, where $\Phi$ and $\Psi$ are the toroidal and poloidal flux enclosed within a flux surface, $\tau_{i i}$ is the ion-ion collision time and $\epsilon=r / a$ is the inverse aspect ratio. Neo-classical transport is therefore expected to be more significant at low toroidal field (and hence at low plasma current $I_{p}$ with a fixed edge safety factor $q_{95}=q\left(\psi_{N}=0.95\right)$ ) and at low heating power with a lower pedestal temperature $\left(\chi_{i, N C} \propto 1 /\left(\sqrt{T_{i}} B_{\phi}^{2}\right)\right)$. Over the power scan represented by the data in Fig. 5 the pedestal collisionality $\nu_{i, p e d}^{\star}$ varies considerably, so an approprate expression for $\chi_{i, N C}$ has to be used valid for all collisionality regimes.

For calculation of $\chi_{i, N C}$ the Chang-Hinton formula [34] is used, which interpolates between collisionality regimes and is appropriate for a large-aspect ratio, shifted circular flux surfaces $\dagger$ We calculate values at the mid-radius of the density pedestal (ETB), assuming that $T_{i}=T_{e}$ and that the mean pedestal ion temperature gradient is half that of the

$\dagger$ We choose not to implement a more accurate calculation using a code such as NCLASS [36] or NEO [37], however, the results of our calculation are in reasonable agreement with the NCLASS calculation of $\chi_{i, N C}$ performed within the interpretive transport code TRANSP [38] in the pedestal region of the JET-ILW pulse \#92432 presented in $\S 4.3$. 
electrons, i.e. $\left\langle d T_{i} / d R\right\rangle_{\text {ped }}=\left\langle d T_{e} / d R\right\rangle_{\text {ped }} / 2$. The total neo-classical loss power is given by $P_{i, N C}=S_{\text {sep }} q_{i, N C}$, where $S_{s e p} \sim 140 \mathrm{~m}^{2}$ is the area of the last-closed flux surface (LCFS).

There are several justifications for making the assumption that $\left\langle d T_{i} / d R\right\rangle_{\text {ped }} /\left\langle d T_{e} / d R\right\rangle_{\text {ped }}=$ $1 / 2$ in the pedestal gradient region. Measurements of the $\mathrm{C}^{6+}$ impurity ion temperature $T_{I}$ over the pedestal region are available for this series of JET-ILW pulses from the edge-CXRS system [40]. These measurements show that, whereas $T_{I} \sim T_{e}$ at the pedestal top, it's gradient $d T_{I} / d R$ is much weaker over the pedestal region, as is that of the $\mathrm{C}^{6+}$ toroidal rotation $\Omega_{\phi}$. The validity of these measurements on JET-ILW plasmas are supported by similar CXRS measurements of both the $\mathrm{C}^{6+}$ and main-ion $\left(D^{+}\right)$ion temperatures in ITER-baseline scenario H-mode plasmas on DIII-D, as shown in Fig. 5 of Ref. [39]. These latter measurements show that the $D^{+}$ion temperature gradient $d T_{i} / d R$ is much stronger than that of the $\mathrm{C}^{6+}$ ion temperature across the ETB, albeit still weaker than $d T_{e} / d R$ (see Fig. 5 of Ref. [39]).

Considering the difficulties with the interpretation of the $\mathrm{C}^{6+}$ ion temperature measurements, we prefer to make the assumption of this fixed ratio of mean ion and electron temperature gradients across the ETB region $\left\langle d T_{i} / d R\right\rangle_{\text {ped }} /\left\langle d T_{e} / d R\right\rangle_{\text {ped }}=1 / 2$. Finally, it should be noted that assumption of a larger ion-electron temperature gradient ratio than $\left\langle d T_{i} / d R\right\rangle_{\text {ped }} /\left\langle d T_{e} / d R\right\rangle_{\text {ped }}=1 / 2$ results in a calculated neo-classical ion heat flux $q_{i, N C}$ that exceeds the measured total inter-ELM heat flux $q_{t o t}$ at high pedestal collisionality $\nu_{i, p e d}^{\star}$, which would be unphysical.

The dependence of the neo-classical component of the pedestal heat transport $P_{i, N C}$ on the total inter-ELM loss power $P_{S e p}^{i E L M}$ is shown in Fig. 6 (a), from which it can be seen that $P_{i, N C}$ is generally less than the total loss power, with both $P_{i, N C}$ and $P_{S e p}^{i E L M}$ increasing with the heating power $P_{a b s}$. In Fig. 6 (b), the ratio of the total heat flux to the neo-classical
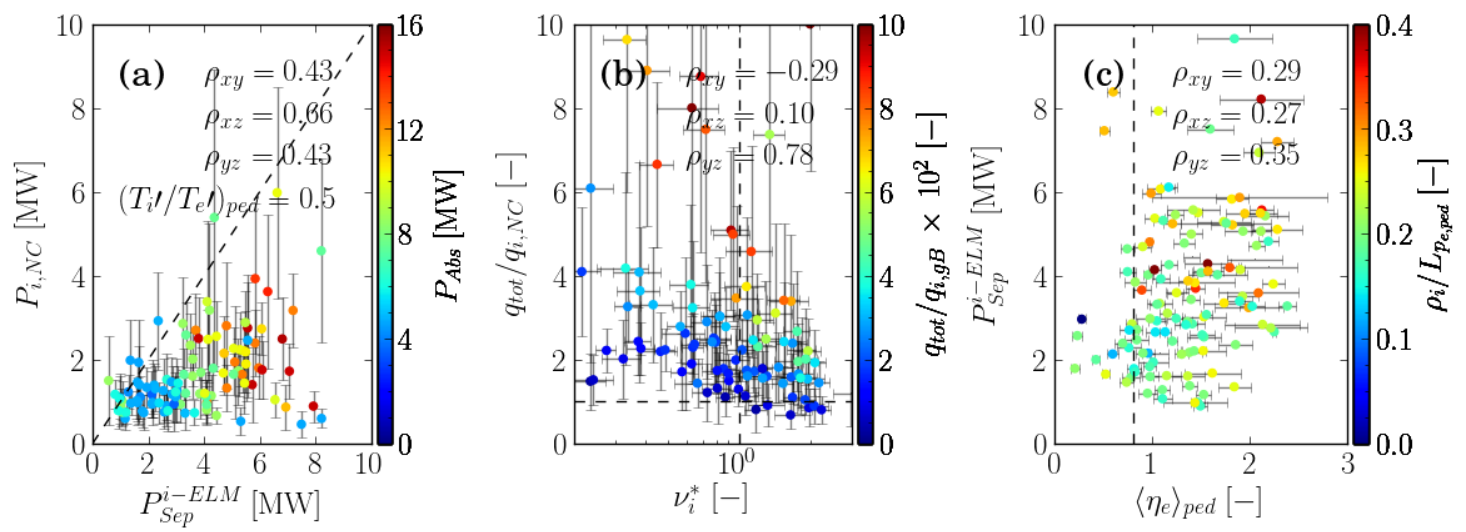

Figure 6: For the 1.4MA/1.7T JET-ILW pulses discussed in §3.1: (a) the neo-classical component of the conducted loss power across the separatrix $P_{i, N C} v$ 's the measured total inter-ELM loss power $P_{S e p}^{i E L M}$; (b) the ratio of the total pedestal heat flux $q_{t o t}=P_{\text {Sep }}^{i E L M} / S_{\text {sep }}$ to the neo-classical ion heat flux $q_{\text {tot }} / q_{i, N C} v$ 's the ion collisionality $\nu_{i}^{\star}$; and (c) the total inter-ELM loss power PSep $P_{\text {SeLM }}$ 's the normalised temperature gradient parameter $\left\langle\eta_{e}\right\rangle_{\text {ped }}$ averaged over the density pedestal (ETB) region. Quantities are evaluated at the mid-radius of the ETB assuming $T_{i}=T_{e}$ and the neo-classical values calculated using the Chang-Hinton interpolation formula for $\chi_{i, N C}$ [34], assuming $\left\langle d T_{i} / d R\right\rangle_{\text {ped }}=\left\langle d T_{e} / d R\right\rangle_{\text {ped }} / 2$. The color scales represent: (a) the absorbed heating power $P_{a b s}$; (b) the total pedestal heat flux normalised to the ion gyro-Bohm level $q_{t o t} / q_{i, g B}$; and (c) a criterion for the efficacy $E \times B$ shear stabilisation in the ETB region $\rho_{i} / L_{p_{e}, p e d}$. Pearson correlation coefficients $\rho_{i j}$ calculated between the $x$-, $y$-and $z$-values (represented by the color scale) of the data points are stated on the plots. 
component $q_{t o t} / q_{i, N C}$ is shown as a function of the ion collisionality $\nu_{i, p e d}^{\star}$ evaluated at the midradius of the density pedestal. At the lowest heating power and hence the highest collisionality $\left(\nu_{i}^{\star}>1\right.$, hence in the collisional Pfirsch-Schlüter (PS) regime), most of the total heat flux is collisional with $q_{t o t} / q_{i, N C} \sim \mathcal{O}(1)$, whilst at the higher heating powers the total heat flux is several times the neo-classical level. This decrease in the relative magnitude of the neoclassical component as $\nu_{i, p e d}^{\star}$ decreases occurs because both $\chi_{i, N C} \propto n_{i} / \sqrt{T_{i}}$ and $\nu_{i}^{\star} \propto n_{i} / T_{i}^{2}$ decrease with $T_{i, p e d}$ and hence with heating power and also because the turbulent component is more strongly driven by a stronger normalised electron temperature gradient $\left\langle\eta_{e}\right\rangle_{\text {ped }}$ across the pedestal (see Fig. $6(\mathrm{c})$ ).

A measure of the strength of the turbulence is the associated turbulent heat flux $q_{t u r b}=q_{t o t}-q_{i, N C}$ normalised to the ion gyro-Bohm level, i.e. $q_{t u r b} / q_{i, g B}$, where the gyroBohm heat flux is given by $q_{i, g B}=-n_{i} \chi_{i, g B} T_{i}^{\prime}$. Here, the ion-gyro-Bohm thermal diffussivity is defined as $\chi_{i, g B}=v_{t h, i} \rho_{i}^{2} / L_{T_{i}} \propto T_{i}^{1 / 2} T_{i}^{\prime}$ (where the ion thermal velocity $v_{t h, i}=\sqrt{2 e T_{i} / m_{i}}$ ) $[30,35]$, implying that the gyro-Bohm heat flux scales as $q_{i, g B} \propto n_{i} T_{i}^{1 / 2} T_{i}^{\prime 2}$. The equivalent gyro-Bohm thermal diffusivity for electron-scale turbulence $\chi_{e, g B}$ is smaller than $\chi_{i, g B}$ by the ratio $\sqrt{m_{e} / m_{i}} \sim 1 / 60$ for deuterium. The motivation in expressing the heat flux relative to the gyro-Bohm level is to normalise out its temperature and density dependence using the simplest physical model for the turbulent heat flux, consistent with the gyrokinetic ordering [41].

In Fig. 6 (b), the colour scale represents $q_{t o t} / q_{i, g B}$, where $q_{i, g B}$ is evaluated at the midradius of the ETB, assuming $L_{T_{i}}=2 L_{T_{e}}$. It can be seen that higher ratios of the total heat flux to the neo-classical component $q_{t o t} / q_{i, N C}$ correspond to more strongly driven turbulence, i.e. to the higher values of $q_{t o t} / q_{i, N C}$ prevailing at higher heating power. Note that the typical values of $q_{t o t} / q_{i, g B} \sim \mathcal{O}(0.01)$ or $q_{t o t} / q_{e, g B} \sim \mathcal{O}(1)$ in electron-gyro-Bohm units.

Here, we compare the measured pedestal $T_{e}$ gradient expressed in terms of $R / L_{T_{e}}$ (or $\eta_{e}$ ) with the threshold behaviour of ETG turbulence, which is expected to be an important channel for heat conduction across the pedestal because it is not as susceptible to suppression by $E \times B$ flow shear as is ITG turbulence [8-11]. Indeed, detailed gyrokinetic calculations are underway of the micro-stability of the pedestal region of these particlar JET-ILW pulses [42]. Preliminary results show ETG instabilities to be dominant across all of these pedestals, while ITG instabilities are expected to be stabilised by the prevailing levels of flow shear.

In order to excite significant heat transport due to ETG turbulence, it is typically required that the $T_{e}$ gradient exceed a critical threshold, i.e. when expressed in terms of $R / L_{T_{e}}$, for this parameter to exceed a critical threshold $R / L_{T_{e}, c r}$. An expression for $R / L_{T_{e}, c r}$ for ETG turbulence is given in Ref. [43]:

$$
R / L_{T_{e}, c r}=\left\{(1+\tau)(1.33+1.91 \hat{s} / q)[1+0.3 \epsilon(d \kappa / d \epsilon)], 0.8 R / L_{n_{e}}\right\}_{\max }
$$

where the magnetic shear $\hat{s}=(r / q)(d q / d r), \tau=Z_{\text {eff }} T_{e} / T_{i}$ and $\kappa$ is the flux-surface elongation. The first of these two expressions corresponds to the 'toroidal' branch of the ETG modes in which drift effects are important $\left(R / L_{n_{e}}<<1\right)$, resulting in a critical $\left(R / L_{T_{e}, c r}\right)^{T}$, which is independent of $R / L_{n_{e}}$, while the second expression corresponds to the 'slab' branch $\left(R / L_{n_{e}}>>1\right)$ in which drift effects are weak, resulting in a critical $\left(R / L_{T_{e}, c r}\right)^{S} \propto R / L_{n_{e}}$ [44]. In the pedestal region, $R / L_{n_{e}} \sim \mathcal{O}(100)$ and is much larger than typical values for $\left(R / L_{T_{e}, c r}\right)^{T}$, which is $\sim \mathcal{O}(10)(\hat{s} / q \leq 4)$. Hence, using the appropriate expression for the slab branch yields an estimate of the critical normalised temperature gradient $\eta_{e, c r}=\left(R / L_{T_{e}, c r}\right)^{S} / R / L_{n_{e}} \sim 0.8$, which has to be exceeded to excite ETG turbulence in the steep-gradient, pedestal (ETB) region.

The dependence of the total inter-ELM loss power $P_{S e p}^{i E L M}$ on the normalised temperature gradient $\left\langle\eta_{e}\right\rangle_{\text {ped }}$ averaged over the ETB region is shown in Fig. 6(c), from which it can be 
seen that this parameter generally exceeds this threshold $\left(\eta_{e, c r}=0.8\right)$ and, for these pulses, typically lies in the range $1<\left\langle\eta_{e}\right\rangle_{\text {ped }}<2$. The electron temperature gradient is hence strong enough to drive significant ETG heat transport. At higher power $\left\langle\eta_{e}\right\rangle_{\text {ped }}$ increasingly exceeds this critical value rather than being rigidly clamped at the threshold.

In non-linear turbulence simulations, e.g. as discussed in Ref. [11], the term 'stiffness' is used to refer to the condition where the turbulent heat flux $q_{t u r b}$, increases faster than linearly with the excess normalised temperature gradient above the critical threshold $\Delta\left(R / L_{T}\right)=$ $R / L_{T}-R / L_{T, c r}$, which in turn implies that the turbulent diffusivity $\chi_{\text {turb }}$ increases with $\left.\Delta\left(R / L_{T}\right)\right)$. Usually, this is investigated in simulations by varying $R / L_{T}$ with other equilibrium parameters held constant. In the limit of infinite stiffness $\left(\chi_{\text {turb }} \rightarrow \infty\right)$, increasing the heat flux causes no further increase of the temperature gradient.

If we assume, for sake of argument, that the pedestal heat transport is infinitely stiff, i.e. it clamps $\eta_{e}$ rigidly to its critical value, this implies that $L_{n_{e}} / L_{T_{e}}=\eta_{e, c r}$, which would be constant across the pedestal. This identity can be expressed as $\eta\left(n_{e}^{\prime} / n_{e}\right)=T_{e}^{\prime} / T_{e}$, which can be integrated inwards from the separatrix $(r=a)$ to yield the relation: $T_{e}(r)=$ $T_{e}(a)\left(n_{e}(r) / n_{e}(a)\right)^{\eta_{e, c r}}$. This implies that, if electron heat transport across the pedestal is very stiff, as it is expected to be if strongly driven at high power, the pedestal temperature will be determined by that at the separatrix $T_{e, s e p}$ and the pedestal density profile according to: $T_{e, p e d}=T_{e, s e p}\left(n_{e, p e d} / n_{e, s e p}\right)^{\eta_{e, c r}}$. This prediction will be discussed further in our comparison of JET-ILW and JET-C pulses in $\S 3.2$.

The data shown in Fig. 4 for this power scan shows that $T_{e, p e d}$ approximately doubles over the factor $\sim 4$ increase in heating power, while there is only a modest $\sim 25 \%$ decrease in $n_{e, p e d}$. The resulting linear increase of $\left\langle d T_{e} / d R\right\rangle_{\text {ped }}$ with the conducted power across the pedestal $P_{S e p}^{i E L M}$ shown in Fig. 5 (b), implies that the effective thermal diffusivity $\left\langle\chi_{e f f}\right\rangle \sim 0.6 \mathrm{~m}^{2} / \mathrm{s}$ is approximately constant with power, so that the pedestal heat transport cannot be particularly stiff. This conclusion is also supported by the data in Fig. 6 (c), which shows that $\left\langle\eta_{e}\right\rangle_{\text {ped }}$ increases with the inter-ELM loss power $P_{S e p}^{i E L M}$ (correlation coefficient $\rho_{x y} \sim 0.3$ ), i.e. $\eta_{e}$ is not strongly clamped to a critical thereshold as it would be if the heat transport were very stiff.

Flow shear may be an important factor in suppressing ion-scale turbulence in the pedestal when the $E \times B$ shearing rate $\gamma_{E}$ exceeds the linear growth rate $\gamma_{l i n}$ of the instabilities. The color scale in Fig. 6 (c) shows that the efficacy of flow shear at suppressing ion scale instabilities, as expressed in terms of $\gamma_{E} / \gamma_{l i n} \sim \rho_{i} / L_{p_{i} \text {,ped }}$ [10], becomes stronger with increasing loss power $P_{l, t h}$ and hence with increasing heating power. While this may act to increasingly suppress any ion-scale turbulence at higher power, the level of flow shear is too small to have any impact on electron-scale turbulence.

In conclusion, we find for these low-current (1.4 MA/1.7 T) JET-ILW pulses that: (i) Ion neo-classical heat transport may be sufficient to explain the inter-ELM loss power at low heating power; (ii) The pedestal heat transport not found to be very stiff over this range of power $\left(\left\langle\eta_{e}\right\rangle_{p e d}\right.$ is not strongly clamped to a critical threshold). This may in part be a result of reduced ion neo-classical heat transport at higher heating power (and hence decreasing $\nu_{i, p e d}^{\star}$ ); and (iii) While $E \times B$ flow shear may play a modest role in regulating ion-scale turbulence, it is too small to have any significant impact on electron scale turbulence, that will be increasingly driven as $\left\langle\eta_{e}\right\rangle_{p e d}$ increases at higher power. 


\subsection{Comparison of high-power, 3.0 MA 'baseline' pulses in JET-C and JET-ILW}

In this section, we compare the loss power components and pedestal parameters in two JETILW and two JET-C 'baseline' scenario pulses at 3.0 MA plasma current with a similar Hmode confinement enhancement factor $H_{98, y} \sim 0.9-1.0$, for which the key parameters are summarised in Table 1. In the JET-ILW pulses, approximately double the heating power is required $\left(P_{a b s} \sim 32 \mathrm{MW}\right)$ to achieve comparable pedestal electron pressure $p_{e, p e d}$ as in the two JET-C pulses $(\sim 15-18 \mathrm{MW})$.

\begin{tabular}{|c||c|c|c|c|c|c|c|c|c|c|c|}
\hline Pulse & Wall & $\boldsymbol{I}_{\boldsymbol{p}}$ & $\boldsymbol{B}_{\boldsymbol{\phi}}$ & $\boldsymbol{q}_{\mathbf{9 5}}$ & $\boldsymbol{\delta}$ & $\boldsymbol{\Gamma}_{\boldsymbol{D}_{\boldsymbol{2}}}$ & $\boldsymbol{P}_{\boldsymbol{a b s}}$ & $\boldsymbol{\beta}_{\boldsymbol{N}}$ & $\boldsymbol{H}_{\mathbf{9 8}, \boldsymbol{y}}$ & $\boldsymbol{f}_{\boldsymbol{E} \boldsymbol{L} \boldsymbol{M}}$ & $\boldsymbol{W}_{\boldsymbol{t h}}$ \\
\hline$\#$ & - & $\mathrm{MA}$ & $\mathrm{T}$ & - & - & $10^{22} / s$ & $\mathrm{MW}$ & - & - & $\mathrm{Hz}$ & $\mathrm{MJ}$ \\
\hline 78677 & $\mathrm{C}$ & 3.0 & 2.4 & 2.6 & 0.24 & - & 17.8 & 1.6 & 1.0 & $15 \pm 1$ & 6.0 \\
78697 & $\mathrm{C}$ & 3.0 & 2.4 & 2.6 & 0.24 & - & 14.7 & 1.6 & 1.0 & $12 \pm 1$ & 5.9 \\
92300 & $\mathrm{Be} / \mathrm{W}$ & 3.0 & 2.8 & 3.0 & 0.2 & 2.5 & 32.1 & 1.8 & 0.9 & $26 \pm 2$ & 7.5 \\
92432 & $\mathrm{Be} / \mathrm{W}$ & 3.0 & 2.8 & 3.0 & 0.2 & 1.9 & 32.0 & 1.9 & 1.0 & $41 \pm 4$ & 8.2 \\
\hline
\end{tabular}

Table 1: Parameters of high-performance JET-C and JET-ILW pulses at $3.0 \mathrm{MW}$ plasma current used for power balance calculations and for which pedestal parameters are shown in Fig. 8. The values of $\beta_{N}$ quoted in this table are calculated using the volume averaged total, thermal pressure $\left\langle p_{t h}\right\rangle$, excluding the fast-ion component, as is appropriate for comparion with the IPB98(y,2) thermal energy confinement scaling [35].

In JET-C pulses gas fuelling was not always necessary during the steady ELMy H-mode phase, both because carbon radiation effectively cooled the divertor plasama, hence reducing the sputtering of the graphite targets, and also because there was sufficient influx of neutrals outgassing from the graphite tiles, as well as the electron source from ionisation of the sputtered carbon influx, to fuel the plasma. In contrast, in high-power JET-ILW pulses, because W impurities radiate predominantly from the hotter, confined plasma, gas fuelling is required to achieve steady $\mathrm{H}$-mode operation with a controlled level of $\mathrm{W}$ impurities. Note that, although retention of deuterium in the graphite wall materials was considerably higher $(\sim \times 20)$ in JET$\mathrm{C}$ than in the metalic (Be/W) JET-ILW walls [47], the rate of deuterium outgassing depends on the temperature of the graphite, can be large and depends upon the previous history of the surface power and gas loading.

In the JET-ILW pulses considered here, the gas puffing rate $\Gamma_{D_{2}}\left(1.8-2.5 \times 10^{22} \mathrm{e} / \mathrm{s}\right)$ was similar to the 'high-gas' fuelling rate used in the gas scans in Ref. [5]. Although a higher gas puffing rate helps to control the influx of sputtered $\mathrm{W}$ from the divertor targets by increasing the ELM frequency $f_{E L M}$, this also degrades the pedestal confinement, reducing the pedestal temperature, which has to be compensated by a higher pedestal density to achieve the same pedestal pressure. Hence, more power is required in JET-ILW to achieve a similar pedestal pressure as in the JET-C pulses, these effects worsening with increasing gas puffing rate [5].

A comparison of the confinement of 2.5 MA baseline-scenario pulses in JET-C and JETILW with matched engineering parameters, including the gas fuelling rates is reported in Ref. [48], where it was also noted that in the JET-ILW pulses $n_{e, p e d}$ was generally somewhat higher and $T_{e, p e d}$ significantly lower than in the JET-C pulses, even though the gas puffing rate was the same, resulting in lower electron confinement overall. The low-triangularity pulses $(\delta \sim 0.22)$, for which kinetic profiles are shown in Fig. 13 (a) of Ref. [48], are at a similar 16.3 MW heating power as the 3MA JET-C pulse \#78677 (without gas puffing) considered here (see Table 1), while the gas puffing rate $\Gamma_{D_{2}} \sim 1.25 \times 10^{22} \mathrm{e} / \mathrm{s}$ was $\sim 70 \%$ of that used in 
the high-power, 3 MA JET-ILW pulse \#92432.

In spite of the lack of direct fuelling in the $3 \mathrm{MA}$ JET-C pulse \#78677, $n_{e, p e d} \sim$ $4 \times 10^{19} \mathrm{~m}^{-3}$ was still $\sim 80 \%$ of that achieved in the $2.5 \mathrm{MA}$ JET-C pulse \#75978 with sustained gas puffing, while $T_{e, p e d} \sim 1.4 \mathrm{keV}$ was similar in both pulses. In contrast, the 2.5 MA JET-ILW pulse \#82779 (with the same gas puffing rate as JET-C pulse \#75978) had $\sim 20 \%$ higher $n_{e, p e d} \sim 6 \times 10^{19} \mathrm{~m}^{-3}$ but much lower $T_{e, p e d} \sim 0.8 \mathrm{keV}$, which is not dissimilar from that achieved $(\sim 0.94 \mathrm{keV})$ at double the heating power in the 3 MA JET-ILW pulse \#92432.

As the JET-C pulses with gas puffing achieve considerably higher $T_{e, p e d}$ than the matched JET-ILW pulses, it is not the strong gas puffing alone that increases the pedestal heat transport in JET-ILW. Other significant differences are: (i) $\mathrm{W}$ radiation in the mantle reducing the temperature in that region and (ii) higher main ion dilution in JET-C compared to that in JET-ILW, which is known to reduce the growth rates of ion scale turbulence [11], alghough other possible causes cannot be excluded, e.g. differences in the recycling properties of the wall materials that might affect the efficacy of gas puff fuelling and hence the structure of the density pedestal.

3.2.1. Comparison of confinement Although these JET-C and JET-ILW pulses have the same $3 \mathrm{MA}$ plasma current, similar pedestal pressures and H-factors $\left(H_{98, y} \sim 1.0\right.$, excluding \#92300 with lower confinement), they are not matched in other respects than the use of gas fuelling only in the JET-ILW pulses. Firstly, the toroidal field in the JET-C pulses (2.4T) was lower than in the JET-ILW pulses $(2.8 \mathrm{~T})$, while the achieved $\beta_{N}$ was higher in the JETILW pulses (1.8-1.9) than in the JET-C pulses (1.6). Both the JET-C and JET-ILW pulses considered here were run with a corner-corner $(\mathrm{C} / \mathrm{C})$ magnetic geometry, with both of the strike points on the horizontal tiles close to the pumping ducts below the vertical targets. For an illustration of a C/C magnetic equilibrium in JET-ILW, see Fig. 14 of Ref. [4].

Retaining only the depedences on the loss power $P_{l, t h}$ and the toroidal field $B_{\phi}$, while holding all other parameters constant in the ELMy H-mode confinement scaling IPB98(y,2) [35], it can be shown that $\beta_{N} \propto H_{98, y} P_{l, t h}^{0.31} / B_{\phi}^{0.85}$ [49], where, $H_{98, y}=\tau_{E, t h} / \tau_{E, s c}$ and $\tau_{E, s c}$ is the confinement time from the IPB98 $(\mathrm{y}, 2)$ scaling. Here, $\beta_{N}$ is calculated using the thermal pressure, i.e. excluding the fast-ion component. It follows that, if the confinement obeys this scaling, it is impossible to achieve the same $\beta_{N}$ at the same $H_{98, y}$ in two, otherwise similar pulses with different heating powers. In fact, the power has to be scaled as $B_{\phi}^{2.74}$, which would already require $52 \%$ more power in the JET-ILW pulses at $2.8 \mathrm{~T}$ than in the JET-C pulses at $2.4 \mathrm{~T}$ to achieve this parity.

We can use this relation to compare the confinement between JET-C and JET-ILW pulses with $H_{98, y} \sim 1.0$, taking account of the change in field and power. Scaling the $\beta_{N} \sim 1.6$ achieved in \#78677 (17.8 MW/2.4 T) to the same $P_{l, t h}$ and $B_{\phi}$ as in \#92432 $(32 \mathrm{MW} / 2.8 \mathrm{~T})$, gives a corrected $\beta_{N^{*}} \sim 1.7$, which is $\sim 10 \%$ lower than actually achieved in the JET-ILW pulse $(\sim 1.9)$. Also, the absolute thermal stored energy $W_{t h}$ in this JET-ILW pulse $(8.2 \mathrm{MJ})$ is almost $40 \%$ higher than in the JET-C pulse (6.0 MJ). As the pedestal pressures are similar between these pulses (see Fig. 8 (c) below), this raises the question of where the additional stored energy resides in the JET-ILW pulse.

Kinetic profiles (from interpretive TRANSP runs using profile fits to the HRTS and CXRS measurements) of both of these pulses are compared in Fig. 7. For the JET-C pulse, the ion dilution is calculated by assuming a constant carbon impurity concentration and matching the calculated line-averaged $Z_{\text {eff }}$ to that measured along a horizontal mid-plane line of sight. For the JET-ILW pulse, time-dependent density profiles of Be, $\mathrm{Ni}$ and $\mathrm{W}$ impurities are used 

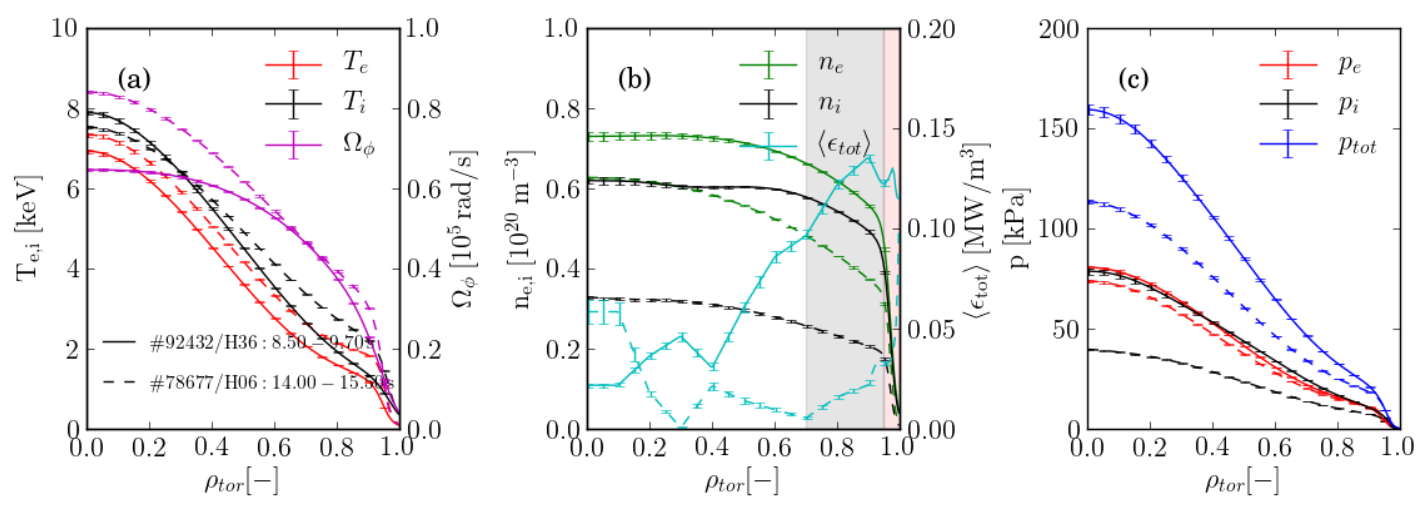

Figure 7: Comparison of fitted kinetic profiles from JET-ILW pulse (\#92432, solid) and JET-C pulse (\#78677, dashed) averaged over their steady H-mode phases showing: (a) electron and ion temperatures $T_{e}$ (red) and $T_{i}$ (black) and toroidal rotation rate $\Omega_{\phi}$ (magenta); (b) electron and ion densities $n_{e}$ (green) and $n_{i}\left(D^{+}\right.$, black) and flux-surface-averaged total emissivity $\left\langle\epsilon_{m}\right\rangle$ (cyan) (c) total, electron and ion pressures $p_{\text {tot }}$ (blue), $p_{e}$ (red) and $p_{i}$ (black); as a function of the normalised radial coordinate $\rho_{\text {tor }}=\sqrt{\Phi_{N}}$, where $\Phi_{N}$ is the normalised toroidal flux enclosed by a flux surface. The shaded bars in (b) indicate the 'mantle' (grey) and 'pedestal' (pink) regions.

as input, which are obtained from an integrated analysis [46] as explained in $\S 4.1$. Coronal ionisation balance is assumed when calculating the charge-state distribution of the impurity ions. Whereas the $\mathrm{C}$ ions are fully ionised in the plasma core, this is not the case for the $\mathrm{W}$ ions that have typical charge states $\mathrm{W}^{+30-50}$ ) and radiate strongly.

For the JET-C pulse with $Z_{\text {eff }} \sim 3-4$, the ion dilution is much more severe $\left(n_{i} / n_{e} \sim 60 \%\right.$, where $n_{i}$ is the density of the $\mathrm{D}^{+}$ions) than in the JET-ILW pulse with lower $Z_{\text {eff }} \sim 1.7$ $\left(n_{i} / n_{e} \sim 80 \%\right)$. As a consquence, in the JET-C pulse, the contribution to the total thermal energy $W_{t h}$ from the electrons $W_{e} / W_{t h} \sim 0.61$ is higher than that from the ions $W_{i} / W_{t h} \sim 0.39$, while in the JET-ILW pulse the electrons and ions contribute almost equally. Note that in both pulses, the ion and electron pedestal pressures are similar, i.e. $p_{i, p e d} \sim p_{e, p e d}$. In the case of the JET-C pulse, although $T_{i, p e d} \sim 1.4 \times T_{e, p e d}$ this is compensated by the lower fractional ion density $n_{i} / n_{e} \sim 0.6$. Therefore, the higher normalised pressure $\beta_{N}$ of these JET-ILW pulses compared to that achieved in the JET-C pulses, e.g. $\beta_{N} \sim 1.9$ in JET-ILW pulse \#92432 compared to $\beta_{N}^{\star} \sim 1.7$ in JET-C pulse \#78677 (corrected for the difference in field and heating power between the two pulses as discussed above), results primarily from a decrease in the ion dilution, which increases the ion pressure and hence the total stored energy in comparison to that in the JET-C pulses.

3.2.2. Comparison of pedestal parameters As shown in Fig. 8 (a-c), the pre-ELM pedestal temperature $T_{e, p e d} \sim 0.7-1.2 \mathrm{keV}$ in the JET-ILW pulses is about half of that in the JET-C pulses $(\sim 1.4-2.0 \mathrm{keV})$, while the pedestal density $n_{e, p e d} \sim 0.6-0.9 \times 10^{20} \mathrm{~m}^{-3}$ is about double in the JET-ILW pulses compared to that in the JET-C pulses $\left(0.3-0.4 \times 10^{20} \mathrm{~m}^{-3}\right)$, resulting in comparable pre-ELM pedestal pressures $p_{e, p e d} \sim 8-12 \mathrm{kPa}$, when compared at the same time after the ELM crash $\Delta t_{E L M}$. Another obvious difference is that in the JET-ILW pulses, as a consquence of the higher power and gas puffing rate, the ELM frequency is much higher (by a factor $\sim 2-3$ ) than in the JET-C pulses. Conversely, the ELM energy losses $\Delta W_{E L M} \sim 0.05-0.25 \mathrm{MJ}$ are much lower in the JET-ILW pulses compared with those in the JET-C pulses (0.2-0.6 MJ). 

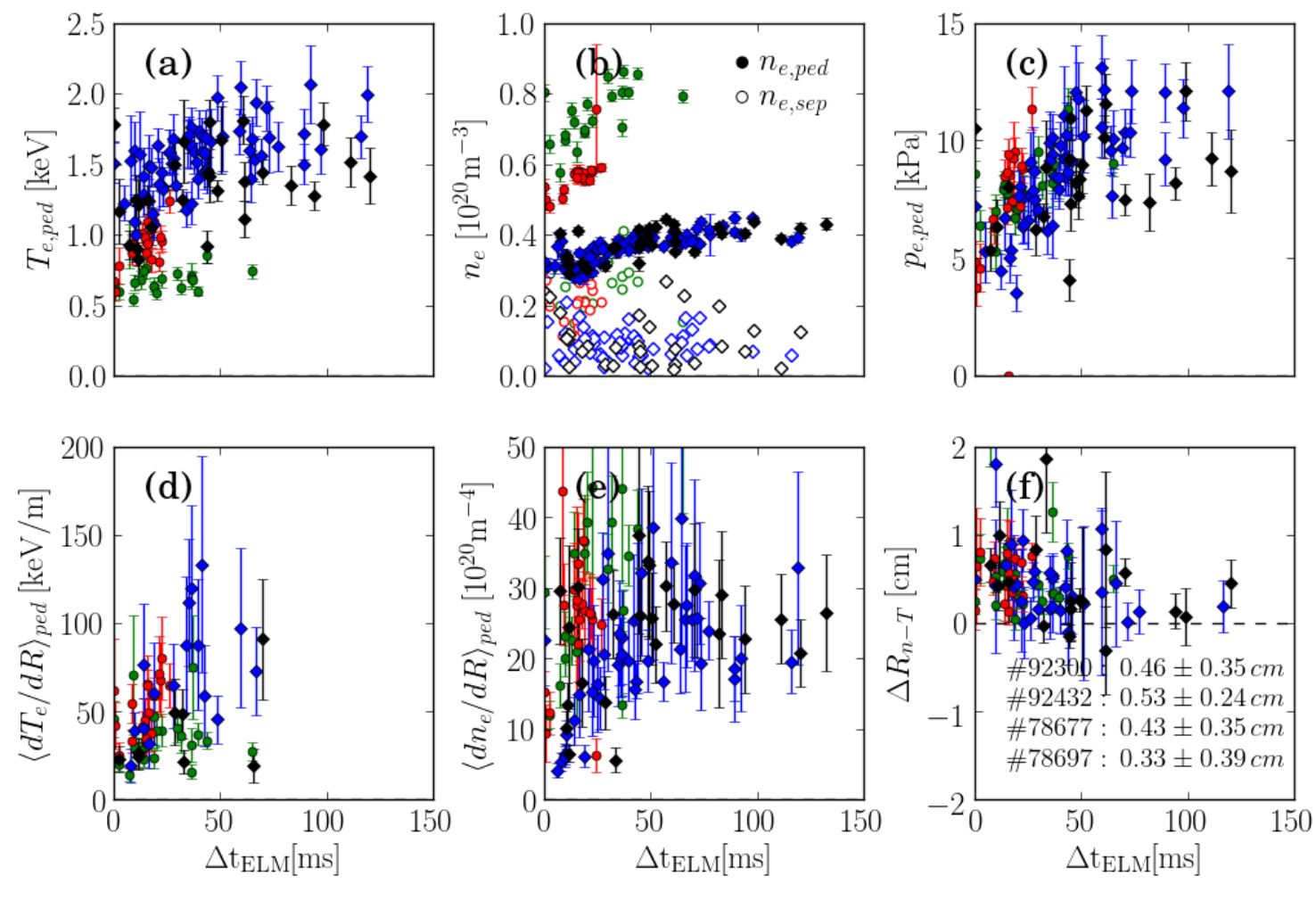

థ $\# 92300$ (ILW, D, $32.1 \mathrm{MW}$ )

§. \#92432 (ILW, D, $33.0 \mathrm{MW}$ )

\#78677 (CW, D, 17.8 MW)

\#78697 (CW, D, 14.8 MW)

Figure 8: The dependence of the pedestal parameters: (a) $T_{e, p e d}$; (b) $n_{e, p e d}(\bullet)$ and $n_{e, s e p}(\circ)$ and (c) $p_{e, p e d}$; the mean radial gradients across the density pedestal (ETB) of (d) $\left\langle d T_{e} / d R\right\rangle_{\text {ped }}$ and (e) $\left\langle d n_{e} / d R\right\rangle_{\text {ped }}$; and $(f)$ the relative shift between the density and temperature pedestal positions $\Delta R_{n-T}$, all as a function of time since the previous ELM $\Delta t_{E L M}$ for the pulses in Table 1.

Averaged values of the pedestal parameters shown in Fig. 8 are given in Table 2 together with the electron pedestal stored energy $W_{e, p e d}=3 / 2 V_{p l} p_{e, p e d}$, where $V_{p l} \sim 80 \mathrm{~m}^{3}$ is the volume of the confined plasma. From this data, it can be seen that the average $\left\langle W_{e, p e d}\right\rangle \sim 0.95 \mathrm{MJ}$ is well matched between the JET-ILW and JET-C pulses. Average values of the ratio of the density at the pedestal top to that at the separatrix $\left\langle n_{e, p e d} / n_{e, s e p}\right\rangle$ are also given in Table 2, where $n_{e, s e p}$ is determined as the density at the location where $T_{e}=100 \mathrm{eV}$. Using this ratio, values of the pedestal temperature, calculated using the simple model for infinitely stiff pedestal heat transport discussed in $\S 3.1 .3$, i.e. $T_{e, p e d}^{\star}=T_{e, s e p}\left\langle n_{e, p e d} / n_{e, s e p}\right\rangle^{\eta_{e, c r}}$ assuming $\eta_{e, c r}=2$ are also stated. The significance of these values of $T_{e, p e d}^{\star}$ are discussed further in $\S 4.2$ below.

Mean gradients of density $\left\langle d n_{e} / d R\right\rangle_{p e d}$ and temperature $\left\langle d T_{e} / d R\right\rangle_{\text {ped }}$ across the ETB, which are calculated from the pedestal height, offset and width, are also shown in Fig. 8 (d-e). Note that the large uncertainites are due to the fact that the resolution of the HRTS system $\Delta R \sim 1-2 \mathrm{~cm}$, which is taken account in the fitting procedure, approaches the scale lengths of the profiles, $L_{n_{e}} \sim L_{T_{e}} \sim 1-4 \mathrm{~cm}$. In the JET-ILW pulses, $\left\langle d n_{e} / d R\right\rangle_{\text {ped }}$ is larger than in the JET-C pulses because $n_{e, p e d}$ is higher but the density pedestal widths $\Delta R_{n_{e}}$ are similar. For the pedestal temperature gradient the comparison is less clear. In spite of the lower $T_{e, p e d}$, at the same time in the ELM cycle, in JET-ILW pulse \#92432 $\left\langle d T_{e} / d R\right\rangle_{p e d}$ is comparable to 
that in the JET-C pulses, while in the other JET-ILW pulse \#92300, $\left\langle d T_{e} / d R\right\rangle_{\text {ped }}$ is lower than in the other pulses, as is $T_{e, p e d}$.

In the case of the JET-ILW pulses, as shown in Fig. 8 (f), there is a clear systematic outward shift of the density pedestal position with respect to that of the temperature pedestal, i.e. $\Delta R_{n-T}=R_{n_{e}, \text { ped }}-R_{T_{e}, \text { ped }} \sim 0.48 \pm 0.25\left(\sigma_{\text {data }}\right) \mathrm{cm}$. For the JET-C pulses, the average relative shift is somewhat smaller $\left(\sim 0.37 \pm 0.3\left(\sigma_{\text {data }}\right) \mathrm{cm}\right)$ than in the JET-ILW pulses, although the scatter is larger. As considered in detail in Ref. [7], a consequence of increasing the relative shift $\Delta R_{n-T}$ between the density and temperature pedestal positions is to increase the parameter $\eta_{e}$, which provides the drive for temperature gradient driven turbulence. As shown in Fig. 12 (c) of $\S 4.2$ below, for the pulses considered here, the mean $\left\langle\eta_{e}\right\rangle_{\text {ped }}$ averaged across the pedestal is somewhat higher in JET-ILW than in JET-C. Note that in JET-ILW this relative outward shift of the density pedestal increases with the gas fuelling rate [6] and is associated with non-peeling ballooning limited pedestals with increasing levels of turbulent pedestal heat transport [7].

Average values of the shear suppression criterion $\rho_{i} / L_{p_{e}, \text { ped }} \sim 0.12-0.23$, which was introduced in $\S 3.1 .3$, are also shown in for these pulses Table 2. These values are comparable to the range of values (0.1-0.3) for the 1.4 MA pulses discussed in $\S 3.1$, which were at the lower toroidal field of $1.7 \mathrm{~T}$. The values are similar because the prevailing values of $\sqrt{T_{i}} / B_{\phi}$ are similar between the two sets of pulses. At least according to this simple criterion, the efficacy of $E \times B$ flow shear at suppressing the level of ion-scale turbulence should be broadly similar for all of these pulses.

\begin{tabular}{|c||c|c|c|c|c|c|c|}
\hline Pulse & $\left\langle\boldsymbol{T}_{\boldsymbol{e}, \boldsymbol{p e d}}\right\rangle$ & $\left\langle\boldsymbol{n}_{\boldsymbol{e}, \boldsymbol{p e d}}\right\rangle$ & $\left\langle\boldsymbol{p}_{\boldsymbol{e}, \boldsymbol{p e d}}\right\rangle$ & $\left\langle\boldsymbol{W}_{\boldsymbol{e}, \boldsymbol{p e d}}\right\rangle$ & $\left\langle\boldsymbol{n}_{\boldsymbol{e}, \boldsymbol{p e d}} / \boldsymbol{n}_{\boldsymbol{e}, \boldsymbol{s e p}}\right\rangle$ & $\left\langle\boldsymbol{T}_{\boldsymbol{e}, \boldsymbol{p e d}}^{\star}\right\rangle$ & $\boldsymbol{\rho}_{\boldsymbol{i}} / \boldsymbol{L}_{\boldsymbol{p}_{\boldsymbol{e}}, \boldsymbol{p e d}}$ \\
\hline$\#-$ & $\mathrm{keV}$ & $10^{20} \mathrm{~m}^{-3}$ & $\mathrm{kPa}$ & $\mathrm{MJ}$ & - & $\mathrm{keV}$ & - \\
\hline 78677 & $1.49 \pm 0.03$ & $0.36 \pm 0.01$ & $7.9 \pm 0.3$ & $0.95 \pm 0.03$ & $4.2 \pm 0.3$ & $1.7 \pm 0.3$ & $0.23 \pm 0.03$ \\
78697 & $1.30 \pm 0.04$ & $0.38 \pm 0.01$ & $8.0 \pm 0.4$ & $0.96 \pm 0.05$ & $3.6 \pm 0.5$ & $1.3 \pm 0.4$ & $0.14 \pm 0.06$ \\
92300 & $0.66 \pm 0.02$ & $0.75 \pm 0.02$ & $7.9 \pm 0.2$ & $0.95 \pm 0.05$ & $2.9 \pm 0.2$ & $0.8 \pm 0.1$ & $0.12 \pm 0.01$ \\
92432 & $0.94 \pm 0.03$ & $0.56 \pm 0.01$ & $7.9 \pm 0.4$ & $0.95 \pm 0.05$ & $3.0 \pm 0.2$ & $0.9 \pm 0.1$ & $0.21 \pm 0.01$ \\
\hline
\end{tabular}

Table 2: Time-averaged values over the steady H-mode phase calculated from the data shown in Fig. 8 of: the parameters $\left(T_{e, p e d}, n_{e, p e d}, p_{e, p e d}\right)$; the electron pedestal stored energy $W_{e, p e d}$; the ratio $\left\langle n_{e, s e p} / n_{e, p e d}\right\rangle$ (determined assuming $T_{e, s e p}=100 \mathrm{eV}$ ); an estimate of $T_{e, p e d}$ from a simple analytic model assuming stiff electron heat transport $T_{e, p e d}^{\star}\left(\right.$ with $\left.\eta_{e}=2\right)$; and the shear suppression criterion $\rho_{i} / L_{p_{e}, \text { ped }}$ discussed in $§ 3.1 .3$.

Results of inter-ELM, power balance calculations for these pulses are shown in Fig. 9, which shows the three loss components for the pulses of Table 3. For the JET-ILW pulse \#92432, the ELM frequency of $41 \mathrm{~Hz}$ is at the limit of reliability for the application of this analysis because the average inter-ELM period $\tau_{E L M} \sim 25 \mathrm{~ms}$ is comparable to the delay between the ELM crash and the minimum of the EFIT $W_{M H D}$ signal. Hence, for this pulse, we show data somewhat earlier from $\Delta t_{E L M} \geq 10 \mathrm{~ms}$ so that the results can at least be compared with those for the other JET-ILW pulse \#92300 in which $f_{E L M} \sim 26 \mathrm{~Hz}$ is lower and the results more reliable.

For these high-power pulses, particularly for those in JET-ILW, the averaged $d W_{M H D} / d t$ component shown in Fig. 9 (b) fluctuates more than for the lower power pulses shown in Fig. 2, consequently, so does the resulting inter-ELM loss power $P_{\text {Sep }}^{i E L M}$. For this reason, for making comparisons with results of gyrokinetic simulations, we quote average values in Table 3 . Note that, although $P_{S e p}^{i E L M}$ data for \#92432 is only available for a short $\sim 10 \mathrm{~ms}$ period, its average magnitude of $\sim 11 \mathrm{MW}$ is comparable to that in the similar pulse \#92300 ( 13 MW). 

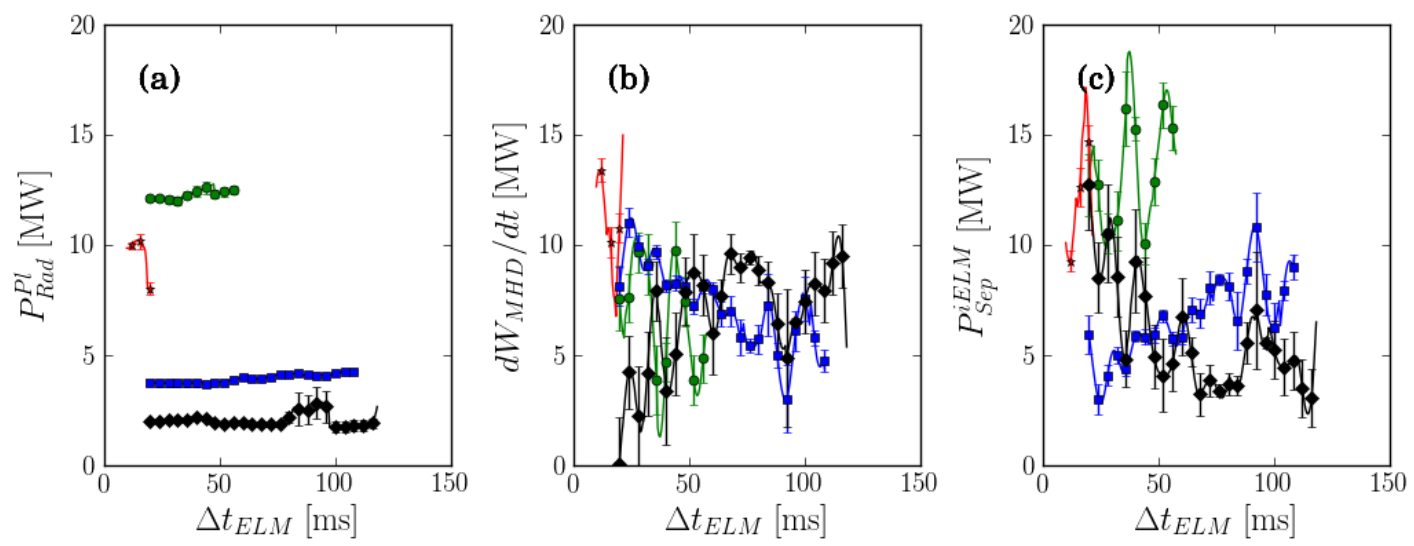

Figure 9: Components of the inter-ELM power balance for the high-power, 3.0 MA JET-ILW (\#92300

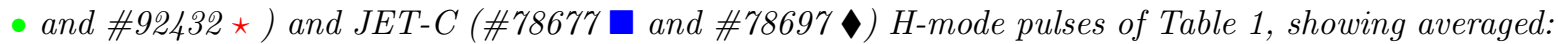
(a) radiated power $P_{R a d}^{P l}$; (b) rate of change of stored energy $d W_{M H D} / d t$; and (c) the inter-ELM loss power $P_{S e p}^{i E L M}$ as a function of time from the previous ELM, $\Delta t_{E L M}$. Average values of each quantity over the inter-ELM period are stated in Table 3.

Results of inter-ELM, power balance calculations for these pulses are summarised in Table 3, which also states the fraction of power $\mathcal{F}_{x}=P_{x} / P_{a b s}$ lost in each channel $x$ relative to $P_{a b s}$. Approximately double the fraction of power $\left(\mathcal{F}_{R a d} \sim 0.3-0.37\right)$ is radiated in these high-power JET-ILW pulses compared to that in the JET-C pulses $(\sim 0.15-0.2)$. Note that at this high input power $(32 \mathrm{MW})$, the radiated power fraction is somewhat higher than in the lower power $(\lesssim 16 \mathrm{MW})$ pulses discussed in $\S 3.1$. The fraction of time-averaged ELM power $\mathcal{F}_{E L M}=\left\langle P_{E L M}\right\rangle / P_{a b s}$ is lower in the JET-ILW $(\sim 0.2-0.3)$ pulses than in the JET-C pulses $(\sim 0.5)$, in spite of the higher ELM frequency in the JET-ILW pulses $(\sim 2-3 \times)$. This is because of the considerably lower ELM energy losses $\Delta W_{E L M} \sim 0.05-0.25 \mathrm{MJ}$ compared to those in the JET-C pulses $(\sim 0.3-0.6 \mathrm{MJ})$.

Although the fraction of power lost due to inter-ELM, pedestal transport $\mathcal{F}_{S e p}=$ $P_{\text {Sep }}^{i E L M} / P_{a b s} \sim 0.3-0.4$ is similar with both walls, in the JET-ILW pulses with twice the heating power, the magnitude of the inter-ELM loss power $P_{S e p}^{i E L M} \sim 11-13 \mathrm{MW}$ due to heat conduction across the pedestal is about twice that in the JET-C pulses $(\sim 6-7 \mathrm{MW})$.

\begin{tabular}{|c||c|c|c|c|c|c|c|c|}
\hline Pulse & Wall & $P_{a b s}$ & $P_{\text {Rad }}^{\text {Pl }}$ & $\left\langle d W_{p l} / d t\right\rangle^{i E L M}$ & $P_{\text {Sep }}^{i E L}$ & $\mathcal{F}_{\text {Rad }}$ & $\mathcal{F}_{E L M}$ & $\mathcal{F}_{\text {Sep }}$ \\
\hline$\#$ & - & MW & MW & MW & MW & - & - & - \\
\hline 78677 & C & $17.85 \pm 0.00$ & $3.79 \pm 0.02$ & $7.29 \pm 0.14$ & $6.77 \pm 0.13$ & 0.21 & 0.50 & 0.29 \\
78697 & C & $14.70 \pm 0.00$ & $2.06 \pm 0.02$ & $6.91 \pm 0.16$ & $5.74 \pm 0.16$ & 0.15 & 0.53 & 0.33 \\
92300 & Be/W & $32.06 \pm 0.05$ & $12.2 \pm 0.05$ & $6.76 \pm 0.31$ & $13.1 \pm 0.28$ & 0.37 & 0.21 & 0.44 \\
92432 & Be/W & $32.02 \pm 0.19$ & $9.42 \pm 0.16$ & $10.90 \pm 0.43$ & $11.7 \pm 0.42$ & 0.30 & 0.33 & 0.38 \\
\hline
\end{tabular}

Table 3: Results of inter-ELM power balance calculations for the JET-C and JET-ILW pulses, where $\mathcal{F}_{x}=P_{x} / P_{a b s}$ is the fraction of power in each loss channel $x$.

The combined effect of the higher fractions of radiated power and inter-ELM pedestal transport in the JET-ILW pulses is therefore to require twice the heating power to achieve the same electron pedestal stored energy $W_{e, p e d}$ as in the JET-C pulses. The higher ELM frequency in the JET-ILW pulses does not, however, result in a higher fraction of ELM loss power due to the smaller amplitude of the ELMs, which are not sufficiently effective to flush 
enough $\mathrm{W}$ impurities from the confined plasma to keep the radiated power fraction as low as in the JET-C pulses. The effect of the high $\mathcal{F}_{R a d}$ in these high-power JET-ILW pulses on the overall power balance of the ions and electrons is discussed in $\S 4$ below.

3.2.3. Comparisons with gyrokinetic simulations of inter-ELM, pedestal transport There has been intensive modelling effort directed towards finding an explanation for the low pedestal temperatures of JET-ILW H-mode plasmas, in particular by the group at the Institute for Fusion Studies (IFS) at the University Texas at Austin [8-11,45]. The fact that twice the heating power and, as our calculations reported here show, twice the heat flux through the pedestal is required to achieve the same $p_{e, p e d}$ but at half of the $T_{e, p e d}$ as in the JET-C comparison pulses, implies a higher heat diffusivity in the pedestal region compared to that in JET-C.

Changes to the edge fuelling in JET-ILW, either due to the necessity for gas puffing and/or a change in the behaviour of recycling neutrals, modifies the pedestal density profile, resulting in a higher normalised pedestal temperature gradient $\eta=L_{n} / L_{T}$, which provides a stronger drive for temperature gradient driven turbulence, at least in the absence of strongly sheared rotation, e.g. driven by beams.

Direct calculations of saturated heat fluxes require non-linear gyrokinetic simulations. Whereas local, flux-tube simulations suffice for the fine-scale ETG turbulence, the larger ionscale (ITG/TEM) turbulence is more faithfully captured by full, global simulations. The most recent of such non-linear simulations using GENE, of both ion and electron-scale turbulence, reported in Ref. [11] are for the same matched JET-ILW and JET-C pulses discussed here in $\S 3.2$ and use the ion temperature profile measured by the CXRS system.

These simulations for the JET-ILW pulse \#92432 predict a total transport power across the ETB in the JET-ILW pulses which compares well with the inter-ELM loss powers reported here (which account for ELM losses as well as radiation), i.e. $12 \mathrm{MW}$ compared to the $33 \mathrm{MW}$ heating power. The components of power conducted across the pedestal by neoclassical transport and ion- and electron-scale turbulence are shown in Fig. 8 of Ref. [11] for various cases. While neo-classical transport carries only $\sim 15 \%$ of the total conducted power, depending on the assumed impurity dilution, ITG turbulence can account for between $\sim 10 \%$ (dominant Be impurity) and $\sim 40 \%$ (dominant $\mathrm{Ni}$ ). By varying $\eta_{e}$ within the experimental uncertainites, the remaining loss power can be explained by the heat flux due to ETG turbulence.

Similar simulations performed for the comparable JET-C pedestal (\#78697, $3 \mathrm{MA} / 2.7 \mathrm{~T}$ at $14.7 \mathrm{MW}$ ) [11] show that the experimental heat fluxes can be explained by a combination of ETG and MTM turbulent and neo-classical heat transport, with ETG accounting for 30\% and neo-classical $\sim 40 \%$ of the total heat flux. In the global, ion-scale simulations, $\beta$ had to be reduced substantially in order to avoid convergence challenges due to problematic modes. The resulting heat flux, which accounts for the remaining $\sim 20 \%$ of the total power, was carried almost entirely by the electrons. In these simulations ITG turbulence was almost completely suppressed, as expected with the low growth rates and high $E \times B$ shear prevailing in JET-C pedestals. Also, MTMs identified in the global, linear simulations exhibited similar flucuation characteristics to washboard MHD modes identified in similar JET-C pulses [50].

It should be noted that earlier simulations by Hatch et al. [8] using GENE for a different JET-ILW equilibrium (\#82585, 2.5 MA/2.7 T) at lower $16 \mathrm{MW}$ heating power, showed that the measured thermal loss power $P_{l, t h}=P_{a b s}-P_{R a d}^{P l} \sim 12 \mathrm{MW}$, i.e. incorrectly including the ELM losses in the conducted power, could be explained by dominant transport in the electron channel, with electromagnetic MTM turbulence contributing two thirds and ETG the 
remainder of the turbulent heat flux.

These simulation results of Hatch et al. suggest that, depending on the particular equilibrium, heat transport in JET-ILW pedestals can be explained by varying relative fluxes in the ion and electron channels, depending on the degree of impurity dilution. In contrast, in JET-C they found the turbulent pedestal heat flux to be predominantly carried by the electrons, with ion-scale turbulence largely suppressed by the stronger $E \times B$ flow shear resulting from the stronger pressure gradient and higher temperature in the JET-C pedestals. Note that in $\S 4$ below, we show that in the JET-ILW pulse \#93432, the ion dilution is primarily due to Be impurities, so the relevant simulations of Ref. [11] consistent with this predict only $\sim 10 \%$ of the total heat flux to be conducted through the ion channel.

\section{Radiation and power balance in high-power, JET-ILW baseline scenario pulses}

In the high-power, JET-ILW baseline pulses discussed here, the fraction of input power lost by radiation $\left(\mathcal{F}_{R a d} \sim 30-40 \%\right)$ is about double that in the JET-C pulses with similar pedestal pressure $\left(\mathcal{F}_{R a d} \sim 15-20 \%\right)$ (see Table 3 ). Such JET-ILW pulses with high heating power $\gtrsim 25 \mathrm{MW}$ typically exhibit a highly asymmetric radiation distribution, which peaks at the low-field side (LFS) periphery of the plasma, which we refer to here as the 'mantle' region $\left(0.7 \leq \rho_{N} \leq \rho_{N, p e d} \sim 0.96\right)$.

A high fraction of radiated power from outside the core confinement region, where the temperature gradient is high, is in principle beneficial as the radiated power is deposited over the full area of the containment vessel. Indeed, proposed scenarios for the DEMO demonstration reactor require a radiated power fraction $\mathcal{F}_{\text {Rad }} \gtrsim 95 \%$ to maintain material heat loads at the targets below $\lesssim 5 \mathrm{MW} / \mathrm{m}^{2}$ [51]. Of course, this is only operationally feasible if the radiating impurities do not migrate into the plasma core, where they can cool the plasma and cause deleterious MHD instabilities.

In $\S 4.1$ below, we show the asymmetric emissivity distribution observed in the high-power JET-ILW pulses to be consistent with the poloidal redistribution of a dominant W impurity within flux surfaces associated with toroidal rotation [52]. Such a strongly asymmetric, mantle radiation is not as evident in the lower power $(\leq 16 \mathrm{MW})$ pulses discussed earlier in $\S 3.1$. Nor is this observed in the JET-C pulses discussed in $\S 3.2$, in which the total radiation is dominated by that from carbon, which is poloidally more symmetric and strongest at the plasma edge and in the scrape-off-layer (SOL) region.

In $\S 4.2$, we discuss the response of the $\mathrm{W}$ transport to profile gradients in the edge region. Generally, in both JET-ILW and JET-C, the temperature and density gradients across the pedestal are aligned such that impurities are convected strongly inwards to the pedestal top. However, we also show that in the sustained phase of the JET-ILW pulses, in the mantle region the direction of this convection is outward, helping to keep the $\mathrm{W}$ impurities out of the core.

The significance of this strong $\mathrm{W}$ radiation from the mantle region in high-performance, JET-ILW pulses in terms of its impact on the overall power balance, the ion and electron heat fluxes across the pedestal and its efficacy in directly cooling the pedestal (ETB) region is investigated in $\S 4.3$.

\subsection{Evidence for $W$ as the dominant radiator}

In high-power, baseline scenario pulses in JET-ILW, tomographic reconstructions of multichord bolometry data typically reveal a highly in-out asymmetric total emissivity distribution, 

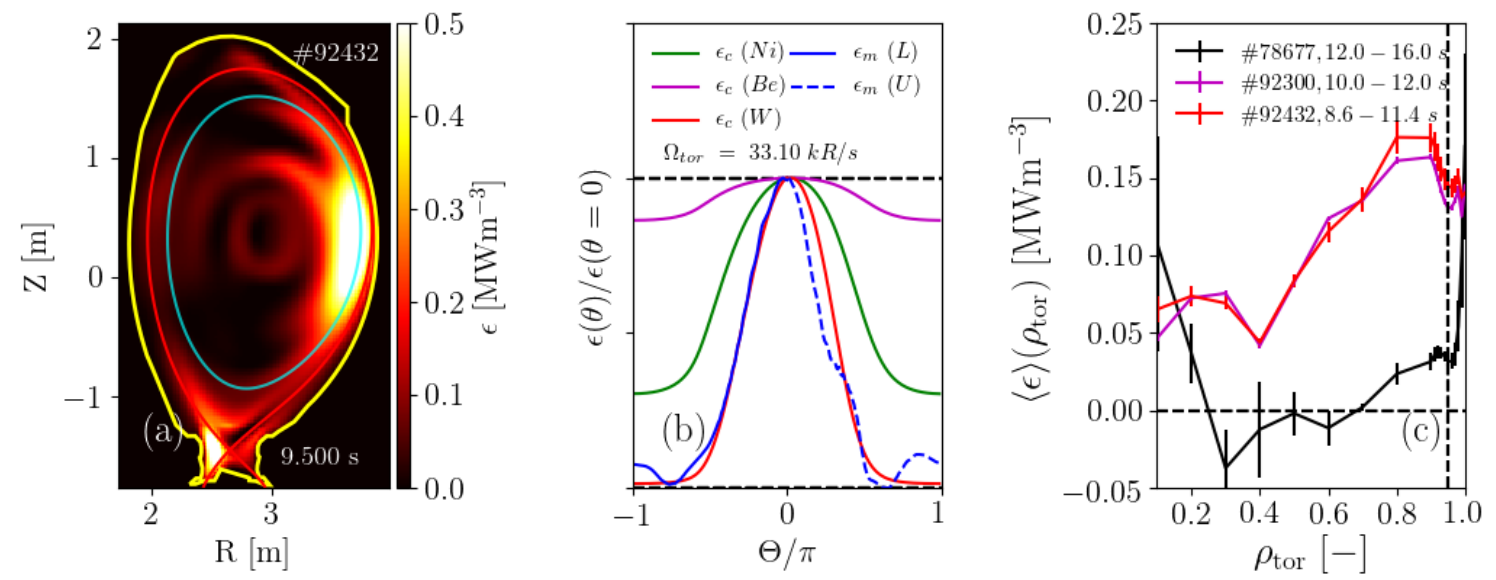

Figure 10: (a) Total emissivity distribution $\epsilon_{m}(R, Z)$ for JET-ILW $3.0 \mathrm{MA}$ pulse \#92432 from bolometer tomography at $9.5 \pm 0.005 \mathrm{~s}$, showing the separatrix in 'red' and the $\psi_{N}=0.8$ flux surface in 'cyan'; (b) normalised measured emissivity $\hat{\epsilon}_{m}(\theta)$ around the $\psi_{N}=0.8$ contour shown in (a) $(\theta>0$ above the magnetic axis) and calculated profiles $\hat{\epsilon}_{c}(\theta)$ for Be (magenta), Ni (green) and $W$ (red) impurities; and (c) flux-surface averaged emissivity profiles $\left\langle\epsilon_{m}\right\rangle\left(\rho_{\text {tor }}\right)$ for three of the pulses in Table 1, including a JET-C pulse for comparison, which doesn't exhibit the strongly asymmetric radiation from the mantle region.

e.g. as shown in Fig. 10 (a) for the 3.0 MA pulse \#92432, which peaks in the mantle region at the LFS mid-plane. The corresponding, flux-surface averaged emissivity $\left\langle\epsilon_{m}\right\rangle$ (shown in Fig. 10 (c) for the two JET-ILW pulses discussed in §3.2), which is relevant for power balance because $T_{e}$ is constant over a flux surface, is hollow and peaks at $\rho_{N} \sim 0.8$, which is well inside the pedestal top $\left(\rho_{N, p e d} \sim 0.96\right)$.

The redistribution of impurity ions $x$ within a flux surface due to toroidal rotation at angular frequency $\Omega_{\Phi}$ has been considered by Wesson in Ref. [53]. Here, we use equations (\#12-20) from Sect. 7 of Ref. [53], which are appropriate for a trace, heavy impurity $x$ in an impure plasma with a single low-Z impurity (here we assume $2 \%$ of Be). The impurity density $n_{x} / n_{x} 0$ is described by Eq. 20 of Ref. [53], which is of the form:

$$
n_{x} / n_{x 0} \propto \exp \left\{\left(1-\frac{T_{e}}{T_{i}-T_{e}} Z_{x} \frac{m_{i}}{m_{x}}\right) \frac{m_{x} \Omega_{\Phi}^{2}\left(R^{2}-R_{0}^{2}\right)}{2 T_{x}}\right\}
$$

where $n_{x 0}$ is the density of the impurity at the outboard mid-plane, i.e. at $R_{0}$ where $\theta=0$, $m_{x}$ and $Z_{x}$ are the impurity mass and charge, $T_{x}$ its temperature and $m_{i}$ is the bulk ion mass. This results from the balance between the electrostatic force due to the gradient of the potential $\varphi$ within the flux surface and the centrifugal force on the ions. When the electron and main ion response is adiabatic $\varphi$ can be determined using the quasi-neutrality condition.

The toroidal rotation rate $\Omega_{\Phi}$ at the particular flux surface is measured using the edge charge-exchange-recombination spectroscopy (CXRS) system observing the $n=11-$ 10, $527.06 \mathrm{~nm}$ transition of extrinsic $\mathrm{Ne}^{+9}$ impurities [40]. For the flux surface shown in Fig. 10 (a) at $\psi_{N}=0.8, \Omega_{\Phi}$ is $\sim 33 \mathrm{krad} / \mathrm{s}$.

The relative total emissivity profile $\hat{\epsilon}_{c}=\epsilon_{c}(\theta) / \epsilon_{c}(\theta=0)$ can be calculated by assuming that radiation from the single, heavy impurity dominates the total emissivity using the expression $\hat{\epsilon}_{c}=\left(n_{x} / n_{x 0}\right)\left(n_{e} / n_{e 0}\right)$, i.e. by assuming a constant $T_{e}$ and a hence constant emission rate coefficient $\mathcal{R}_{\epsilon}\left(T_{e}\right)$ over the flux surface. 
A comparison of the calculated relative emissivity distributions $\hat{\epsilon}_{c}$ for the heavy impurities $\mathrm{Ni}\left(M_{x}=n_{x} / n_{p} \sim 59\right), \mathrm{W}\left(M_{x} \sim 184\right)$ and for Be $\left(M_{x} \sim 9\right)$ to the measured normalised total emissivity $\hat{\epsilon}_{m}$ is shown in Fig. 10 (b). Clearly, in this high-power JET-ILW pulse \#92432, the measured distribution at this flux surface is consistent with the assumption that the total emissivity is dominated by radiation from $\mathrm{W}$ impurities - the validity of this assumption is discussed next.

Using atomic data from ADAS [54] and assuming coronal ionisation balance, in the mantle region in which $T_{e} \sim 1-2 \mathrm{keV}$, the dominant charge states of the $\mathrm{W}$ impurities are expected to be $\mathrm{W}^{+25-30}$, for which the total emissivity coefficient $\mathcal{R}_{\epsilon}$ is approximately constant at $\sim 4.5 \times 10^{-31} \mathrm{Wm}^{3}$. This is corroborated by the observation of emission bands from $\mathrm{W}^{+23-26}$ ions in the regions of $19 \pm 1$ and $29 \pm 1 \mathrm{~nm}$ in VUV spectra viewing horizontally through the region of strong emission at the LFS mid-plane. Assuming this value of $\mathcal{R}_{\epsilon}$ and an average value of $n_{e} \sim 6 \times 10^{19} \mathrm{~m}^{-3}$, the peak and flux-surface-averaged (in brackets) values of total emissivity $\epsilon_{m}$ in the mantle region of $0.6(0.2) \mathrm{MWm}^{-3}$ respectively correspond to $\mathrm{W}$ concentrations of $n_{W} / n_{e} \sim 4.0(1.2) \times 10^{-4}$. These concentrations contribute modestly to the effective ion charge $\Delta Z_{\text {eff }} \sim 0.4(0.12)$ and insignificantly to the plasma mass density $\Delta \rho_{M} \sim 3(1) \%$, in spite of the $\mathrm{W}$ radiating $\sim 40 \%$ of the $32 \mathrm{MW}$ input power.

Data from ADAS for these impurities [55] show that the total emission coefficient for $\mathrm{W}$ over the temperature range $1-10 \mathrm{keV}$ is $\gtrsim 50$ times higher than either Fe or Ni. Hence, radiation from $\mathrm{W}$ is expected to completely dominate that from other impurities. This is confirmed by the results of a much more extensive, combined analysis of line-integrated, multi-channel measurements of soft X-ray (SXR) emission and bolometric total radiation and horizontal mid-plane visible bremsstrahlung $Z_{\text {eff }}$ measurements. This analysis is an extension of that described in Ref. [46] to incorporate the total radiation and $Z_{\text {eff }}$ measurements together with the SXR data self-consistently.

Results of this analyis are shown in Fig. 11, which shows profiles of impurity
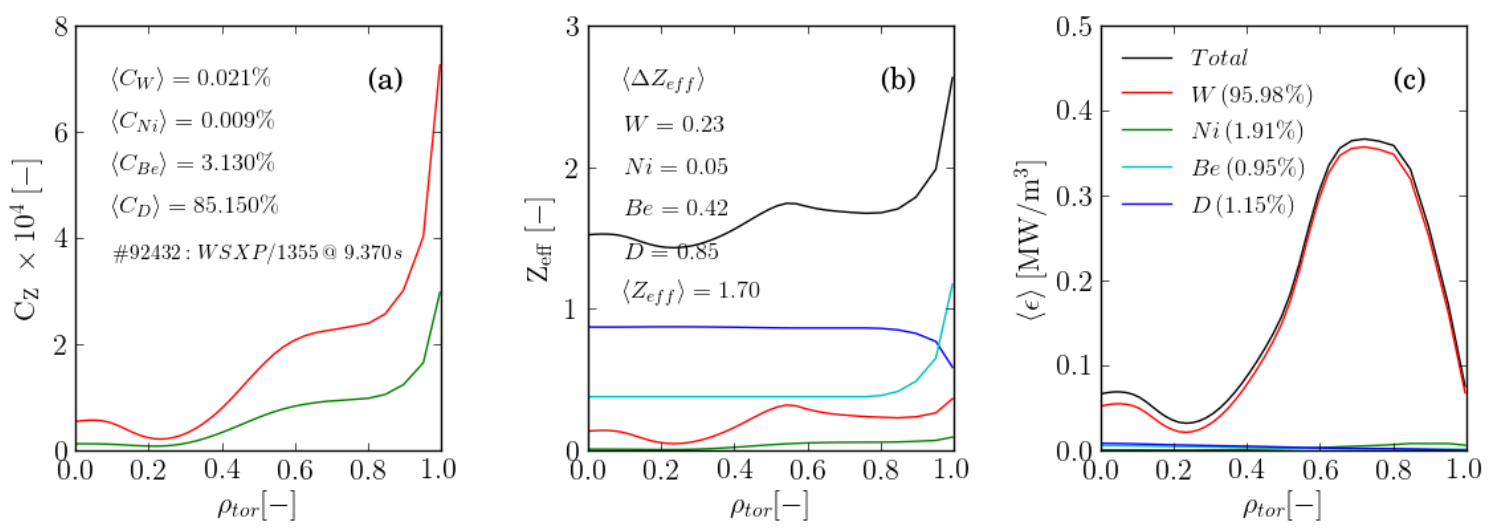

Figure 11: Results of integrated analysis of $S X R$, bolometer and line-averaged $Z_{\text {eff }}$ data at $9.37 \mathrm{~s}$ during the steady H-mode phase of \#92432 showing profiles of: (a) concentrations $C_{Z}=n_{Z} / n_{e}$ of Ni and $W$ impurities $\left(C_{B e} \sim 3.1 \%\right.$ and $C_{D} \sim 87.6 \%$ are constant with $\left.\rho_{\text {tor }}\right)$; (b) total $Z_{\text {eff }}$ and $\Delta Z_{\text {eff }}$ from each ion species; and (c) flux-surface averaged emissivities $\langle\epsilon\rangle$ (total and from each ion species) as a function of $\rho_{\text {tor }}$. Volume averaged quantities are stated as $\langle\ldots\rangle$ in the legend. The colour key is the same for plots $(a-c)$. Note that the corresponding $2 D(R, Z)$ emissivity distrubutions and the measured and reconstructed line-integrated intensities for the SXR and bolometer cameras are shown in Fig. C1 of $\S$ Appendix C for completeness. 
concentrations $C_{Z}=n_{Z} / n_{e}(\mathrm{~W}, \mathrm{Ni}$ and $\mathrm{Be})$ and their individual contributions $\Delta Z_{\text {eff }}$ to the total $Z_{\text {eff }}$ and to the total emissivity $\left\langle\epsilon_{m}\right\rangle$. While the Be concentration is assumed radially constant, the in/out assymmetry of the $\mathrm{Ni}$ and $\mathrm{W}$ concentrations are forced to be consistent with the torodial rotation rate $\Omega_{\phi}$ according to Eq. (3). From this it can be seen that, while the resulting $\mathrm{W}$ concentration $(\sim 0.021 \%)$ is only twice that of $\mathrm{Ni}$, the $\mathrm{W}$ contributes $\sim 96 \%$ of the volume-averaged, total emissivity $\left\langle\epsilon_{m}\right\rangle$. Hence, the results of this much more sophisticated analysis support the main conclusion of the simplified analysis presented above, i.e. that radiation from the $\mathrm{W}$ impurities dominates the total emissivity.

Analysis of bolometer data for the 1.4 MA JET-ILW pulses discussed in $\S 3.1$ above does not reveal the presence of similarly strong $\mathrm{W}$ radiation from the mantle region in these lower power pulses $\left(P_{a b s}=4.5 \rightarrow 16 \mathrm{MW}\right)$ with the lower rate of gas fuelling. In these pulses, the radiated power fraction decreases $\left(\mathcal{F}_{R a d}=0.35 \rightarrow 0.25\right)$ with increasing input power. Note that the average emissivity profile $\left\langle\epsilon_{m}\right\rangle$ for one of the JET-C pulses is shown in Fig. 10 (c) in which it can be seen that the radiation, which is predominantly from $\mathrm{C}$, is emitted most strongly from the SOL, although there is also sign of some accumulation of high-Z impurities in the core.

\subsection{Response of $W$ and heat transport to edge gradients}

The fits to the HRTS $T_{e}$ and $n_{e}$ profile data also yield an estimate of the linear, core gradient from which we can calculate mean values of $R / L_{T_{e}}$ and $R / L_{n_{e}}$ in the mantle region $\left(0.7 \leq \rho_{N} \leq \rho_{N, p e d}\right)$. These estimates can be used to calculate the parameter $\zeta_{N C} \equiv R / 2 L_{T_{e}}-R / L_{n_{e}}$, where the scale lengths $L_{n, T}$ are positive for a peaked profile. Assuming $T_{e}=T_{i}, \zeta_{N C}$ is proportional to the neo-classical, radial pinch velocity of the impurities, i.e. $R V_{N C} \propto Z q^{2} D_{c} \zeta_{N C}$, where $D_{c}=\rho_{i}^{2} / \tau_{i i}$ is the classical diffusion coefficient and $V_{N C}$ is radially outwards for $\zeta_{N C}>0$ (the full expression for $V_{N C}$ is given by Eq. 2 of Ref. [59]). The neo-classical convection is hence stronger for the highly charged $\mathrm{W}$ impurities with which we are concerned. Furthermore, in Ref. [19], it is shown that a strong outward assymmetry of the impurity density enhances the neo-classical convection by the factor $1 /\left(2 \epsilon^{2}\right) \sim 5$, where $\epsilon=r / R$ is the inverse aspect ratio.

Similarly, from the fitted profiles we also calculate mean values of $\zeta_{N C}$ and $\eta_{e}$ across the mantle region, $\left\langle\zeta_{N C}\right\rangle_{m a n}$ and $\left\langle\eta_{e}\right\rangle_{m a n}$, and mean values of both $\zeta_{N C}$ and $\eta_{e}$ over the full width of the density pedestal, $\left\langle\zeta_{N C}\right\rangle_{p e d}$ and $\left\langle\eta_{e}\right\rangle_{\text {ped }}$. The results of this analysis are shown in Fig. 12 for the pulses considered in $\S 3.2$, which reveals some striking differences in these parameters in the mantle region between the JET-ILW and JET-C pulses.

Considering first the pedestal (ETB) region, at the same time in the ELM cycle $\Delta t_{E L M}$, the mean values $\left\langle R / L_{T_{e}}\right\rangle_{\text {ped }}$ and $\left\langle R / L_{n_{e}}\right\rangle_{\text {ped }}$ over the ETB region are quite similar, although there is a large scatter, which result in values of $\eta_{e} \lesssim 2$, which are on average higher in the JET-ILW pulses than in the JET-C pulses. Hence, we might expect temperature gradient driven turbulent transport to be more virulent in the ETB region of the JET-ILW pulses, a hypothesis consistent with the higher inter-ELM loss power $P_{S e p}^{i E L M}$ found from our power balance analysis.

We can compare the average pedestal temperature $\left\langle T_{e, p e d}\right\rangle$ with the predicted temperature for stiff pedestal heat transport using the expression that was introduced in $\S 3.1 .3: T_{e, p e d}^{\star}=$ $T_{e, s e p}\left\langle n_{e, p e d} / n_{e, s e p}\right\rangle^{\eta_{e, c r}}$. Here, we take the average value of $\left\langle n_{e, p e d} / n_{e, s e p}\right\rangle$ (determined by assuming $T_{e, s e p}=100 \mathrm{eV}$ ) and taking the value $\eta_{e, c r}=2$, which is the approximate value at which $\left\langle\eta_{e}\right\rangle_{\text {ped }}$ appears to saturate, particularly in the two JET-ILW pulses. As can be seen from Table 2, the prediction is in reasonable agreement (within the uncertainties) with the 

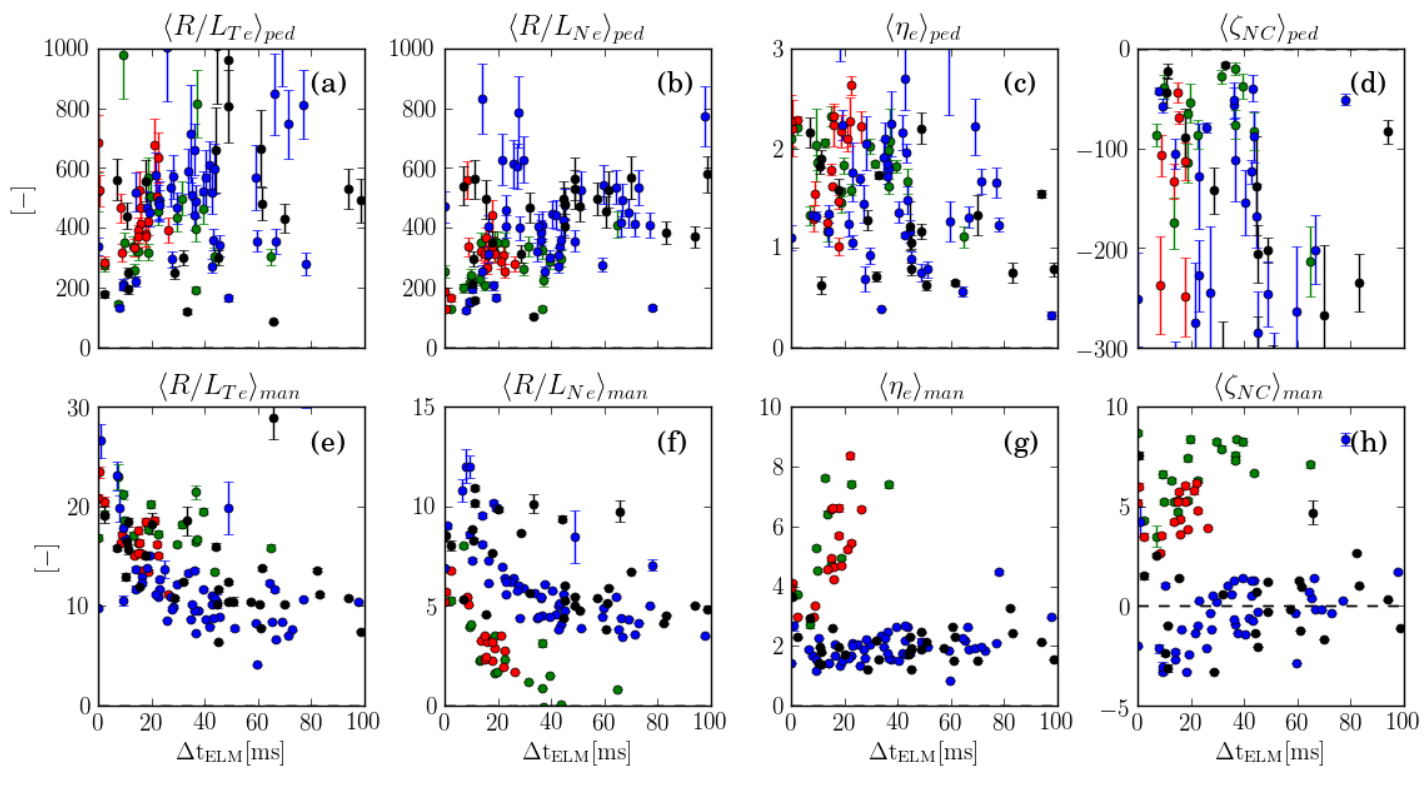

§. \#92300 (ILW, D, 32.1 MW)

§ \#92432 (ILW, D, $33.0 \mathrm{MW})$

Ф $\# 78677$ (CW, D, $17.8 \mathrm{MW})$

1. \#78697 (CW, D, $14.8 \mathrm{MW})$

Figure 12: Average values over the pedestal gradient (ETB) region $\langle\ldots\rangle_{\text {ped }}$ (top) and the mantle region $\langle\ldots\rangle_{\operatorname{man}}$ (bottom) of the gradient parameters: $(a, e) R / L_{T_{e}} ;(b, f) R / L_{n_{e}} ;(c, g) \eta_{e}$; and $(b, f) \zeta_{N C}$ as a function of the time since the previous ELM $\Delta t_{E L M}$ for the JET-ILW and JET-C pulses of Table 1.

actual mean pedestal temperatures $\left\langle T_{e, p e d}\right\rangle$. This agreement indicates that: (i) electron (or ion) temperature gradient driven transport, driven by $\eta_{e}$ (or $\eta_{i}$ ), dominates the anomalous heat transport in the pedestal and (ii) that the reduced pedestal density gradient in these JET-ILW pulses with a high level of gas puffing, is the cause of the lower pedestal temperature compared to that in the JET-C pulses. Note that recent high-power pulses in JET-ILW without gas fuelling during the ELMy H-mode phase exhibit a much higher pedestal temperature [56].

It is the net impurity influx across the separatrix, resulting from a balance between inward convection through the ETB and expulsion by the ELMs ( $a k a$ 'flushing'), which governs the evolution of the impurity content. Between ELMs, the pedestal $T_{e}$ and $n_{e}$ gradients result in large negative values of $\zeta_{N C} \sim \mathcal{O}(10-100)$ such that $\mathrm{W}$ impurities (which are sputtered at the targets and migrate up the SOL) are driven through the ETB to the top of the pedestal. Note that in these high-performance JET-ILW pulses $\left|\zeta_{N C}\right|$ is on average smaller than in the JET-C pulses, primarily because $R / L_{n_{e}}$ is on average smaller, but not sufficiently so to reverse the direction of convection and thereby keep the impurities out of the confined plasma.

Turning now to the mantle region, there are stark differences in the normalised gradients between the pulses with the different walls. While in the JET-ILW pulses $\left\langle R / L_{T_{e}}\right\rangle_{\text {man }} \sim 15-20$ is somewhat higher than in the JET-C mantle, where $\left\langle R / L_{T_{e}}\right\rangle_{\text {man }} \sim 10-15,\left\langle R / L_{n_{e}}\right\rangle_{\text {man }}$ is much lower. Whereas in JET-C, $\left\langle R / L_{n_{e}}\right\rangle_{\text {man }}$ evolves during the ELM cycle from $\sim 10 \rightarrow 5$, it decreases to much smaller values $\left(\left\langle R / L_{n_{e}}\right\rangle_{\text {man }} \sim 5 \rightarrow 2\right)$ in the JET-ILW pulses as the density profile flattens. Consequently, $\left\langle\eta_{e}\right\rangle_{\text {man }}$ is much higher in the JET-ILW mantle than in the JET-C pulses, increasing during the ELM cycle $\left(\eta_{e} \sim 2 \rightarrow 10\right)$ due to the weakening of the density gradient. In contrast, in the JET-C mantle, $\left\langle R / L_{T_{e}}\right\rangle_{\text {man }}$ and $\left\langle R / L_{n_{e}}\right\rangle_{\text {man }}$ evolve in a similar manner, resulting in a quite constant value of $\left\langle\eta_{e}\right\rangle_{\text {man }} \sim 2$.

It is suggested that this difference in the evolution of $\left\langle\eta_{e}\right\rangle_{\text {man }}$ between JET-C and JET- 
ILW might be explicable in terms of the different dependencies on the normalised density gradient $R / L_{n_{e}}$ of the threshold behaviour of the slab or toroidal branches of ETG turbulence, which might be responsible for stiff electron heat transport in the mantle region. The expression given by Eq. (2) for the critical gradient $R / L_{T_{e}, c r}$ for ETG turbulence, introduced in $§ 3.1 .3$ [43], predicts different behaviour depending on the strength of the density gradient. In the JET-ILW mantle, where the density gradient is weaker $\left(\left\langle R / L_{n_{e}}\right\rangle_{\text {man }} \sim 5 \rightarrow 2\right)$, the first expression for the toroidal branch is more applicable, predicting a constant $\left(R / L_{T_{e}, c r}\right)^{T} \sim 4$. Although the observed values $\left(\left\langle R / L_{T_{e}}\right\rangle_{\text {man }} \sim 15-20\right)$ do remain quite constant, these are well above the predicted threshold value. In contrast, in the JET-C mantle, where the density gradient is stronger, the second expression is applicable, i.e. $\left(R / L_{T_{e}, c r}\right)^{T} \sim 5 \lesssim$ $\left(R / L_{T_{e}, c r}\right)^{S}=0.8 R / L_{n_{e}} \sim 4-8$. Hence, a constant $\eta_{e, c r} \sim 0.8$ is applicable, which is none the less somewhat below than the constant observed value of $\left\langle\eta_{e}\right\rangle_{\text {man }} \sim 2$. Further, detailed gyrokinetic calculations of the micro-stability of the mantle region for these equilibria are certainly required to test this hypothesis.

Interpretive transport analysis of the JET-ILW pulse \#92432, discussed in $§ 4.3$ below, shows that the electron thermal diffusivity is higher than that of the ions in the mantle region, i.e. $\chi_{e} / \chi_{i} \lesssim 2$ (see Fig. $15(\mathrm{c})$ ), suggesting the presence of strong ETG driven turbulence. Note that non-linear micro-stability calculations have been performed for the mantle regions, just inside the pedestal top, for two JET-ILW H-mode plasmas with matched pedestals at high and low rates of gas fuelling - very different heating powers were required to match the pedestal pressure in these plasmas [7]. It is found that $\eta_{e}$ is considerably higher in the mantle of the pulse with the higher level of gas fuelling, which enhances the linear growth rates of ETG modes. It was also found that $\gamma_{E}<<\gamma_{\text {lin }}^{E T G}$ but that $\gamma_{E}$ should be sufficient to suppress ITG modes over much of the $k_{y} \rho_{i}$ spectrum, especially in the pulse with the lower level of gas fuelling. Further detailed gyrokinetic calculations are required to investigate the heat transport in the edge regions of these JET pulses in more detail.

In the mantle of the JET-ILW pulses, the resulting positive values of $\zeta_{N C} \sim 5-10$ inhibit migration of the impurities into the plasma core, which is consistent with the hollow total emissivity profile shown in Fig. 10 (c). In contrast, due to the stronger mantle density gradient in the JET-C pulses, the values are smaller $\left|\zeta_{N C}\right| \lesssim 3$ and sometimes negative. Hence, in JET-C pulses, the impurities are less well screened from the plasma core, for instance some degree of accumulation of presumably high-Z impurities is observed in pulse \#78677 (see Fig. 7 (b)). Note that later in the JET-ILW pulse \#92432 at $10.2 \mathrm{~s}$, after the ELMs cease, $\zeta_{N C}$ suddenly changes sign $<0$ in the mantle, causing the $\mathrm{W}$ (and any other high-Z impurities) to accumulate in the plasma core. These changes are caused by an H/L-transition at the end of an ELM-free period (at $\sim 10.5 \mathrm{~s}$ ), during which the radiated power fraction increases to $\mathcal{F}_{\text {Rad }} \sim 70 \%$ because of the increasing density. The influence of the strong W impurity radiation on the evolution and performance of high-power ITER-baseline and 'hybrid' pulses in JET-ILW [68] is to be addressed in a future publication [57].

An explanation of the difference in the density profiles between JET-ILW and JET-C might be related to the effective electron collisionality $\nu_{e f f} \propto n_{e} / T_{e}^{2}$ [60], which is almost an order of magnitude higher at the top of the JET-ILW pedestals than in JET-C and is known to affect particle transport. In Ref. [4], analysis of the density peaking in low-current, low-triangularity, JET-ILW pulses, including those discussed in $\S 3.1$, shows that the peaking decreases with increasing pedestal collisonality. Also, in JET-C H-mode pulses, the density peaking defined as $n_{e, 0} / \overline{n_{e}}$ (where $n_{e, 0}$ is the density at the magnetic axis and $\overline{n_{e}}$ the lineaveraged density) was found to decreases with $\nu_{e f f}[61]$. 


\subsection{Interpretive transport analysis}

In this section, the effect of the strong $\mathrm{W}$ radiation from the mantle region in the high-power, JET-ILW pulse \#92432 on the power balances of the ions and electrons is investigated by means of interpretive transport analysis using TRANSP $[38,62]$. The primary input data for the transport analysis are the kinetic profiles $\left(T_{e}, n_{e}, T_{i}\right.$ and $\left.\Omega_{\Phi}\right)$, which are obtained by fitting the raw data from HRTS and the edge and core CXRS systems, e.g. as shown in Fig. 7. The fits use an mtanh() function to represent the pedestal region [27] and a third-order polynomial in the core. Examples of these profiles at $9.5 \mathrm{~s}$ are shown in Fig. 13 (a). As the $T_{i}$ data from the edge CXRS system on JET-ILW is considered more reliable than from the core system, the outer two points of the core CXRS (not shown) are excluded from the fit.

Pressure constrained EFIT ++ equilibrium reconstructions are used to map the measurement data from major radius to the flux coordinate, i.e. $\rho_{t o r}\left(R_{m}\right)$ ( where $\rho_{t o r}=\Phi_{N}^{1 / 2}$ and $\Phi_{N}$ is the normalised toroidal flux). An additional, weakly-weighted constraint on the separatrix temperature to ensure $T_{e, s e p} \sim 100 \mathrm{eV}$ is also applied to ensure the separatrix is sufficiently well aligned with respect to the edge measurements to obtain meaningful results from the interpretive transport calculations in the pedestal region (see Ref. [29,42] for further justification of this procedure).

To ensure the correct dilution and $Z_{\text {eff }}$ profiles are taken into account, time-dependent density profiles of the Be, $\mathrm{Ni}$ and $\mathrm{W}$ impurities (obtained from the integrated analysis [46] as explained in $\S 4.1$ above), which correspond to the data shown in Fig. 11, are also input to the TRANSP calculations.

For the readers who are unfamiliar with interpretive transport analysis, the various terms in the electron and ion power balances are defined in $§$ Appendix B. Note that the transport loss powers (sum of convective and conductive components) in the ion and electron channels are denoted in the following by $P_{\text {tran,i } i}$ and $P_{\text {tran,e }}$, while the total transport loss power is denoted as $P_{\text {tran }}=P_{\text {tran }, e}+P_{\text {tran }, i}$. If the results of the TRANSP analysis are averaged over many ELM cycles (or performed using ELM-averaged input profiles), at the separatrix, $P_{\text {tran }}$ is equivalent to the sum of the inter-ELM loss power and the time-averaged ELM loss powers from our earlier analysis of $\S 2.3$, i.e. $P_{t r a n} \equiv P_{S e p}^{i E L M}+\left\langle P_{E L M}\right\rangle$.

4.3.1. Power balance during sustained ELMy phase A detailed study of thermal transport in baseline scenario JET-ILW plasmas presented in Ref. [63] concludes that in these highpower pulses, the ions are hotter than the electrons in the core because of: enhanced ion heating at high heating power (due to the higher relative fraction beam heating to the ions $P_{N B, i} /\left(P_{N B, i}+P_{N B, e}\right)$, which increases with the ratio of $T_{e}$ to the injection energy $\left.T_{e} / E_{0}\right)$, a reduction in the ion thermal transport due the higher $T_{i} / T_{e}$ partially stabilising ITG turbulence and a possible role of $E \times B$ flow shear stabilisation.

Referring to the results of the power balance calculations during the sustained ELMy phase of pulse \#92432, as shown in Fig. 13, we can see immediately that substantial power $P_{e i} \sim 9 \mathrm{MW}$ is transfered from the ions to the electrons, which largely equilibrates $T_{e}$ and $T_{i}$ near the pedestal top, where the total electron loss power (including ionisation losses and radiation) $P_{\text {loss }, e} \sim 20 \mathrm{MW}$ is twice the total ion loss power (including charge-exchange losses) $P_{\text {loss }, i} \sim 12 \mathrm{MW}$. Note that here we quote values at the pedestal top $\left(\rho_{\text {tor }} \sim 0.96\right)$ rather than at the separatrix. The strong radiation from the mantle region cools the electrons (radiation is a loss term in the electron power balance) and hence acts to increases the magnitude of ion-electron exchange power in this region.

In TRANSP the net charge-exchange loss power $P_{C X}$ is calculated using a $1.5 \mathrm{D}$ neutral 

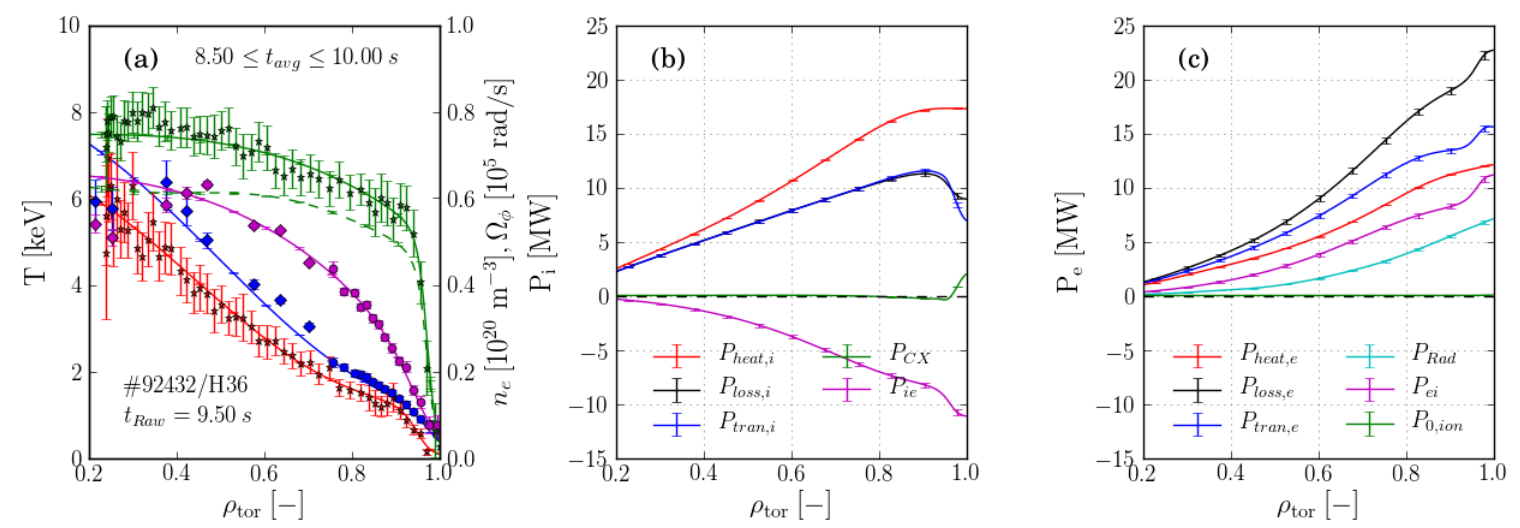

Figure 13: Results of interpretive, TRANSP power balance analysis of JET-ILW pulse \#92432 showing: (a) kinetic profiles $T_{e}$ (red), $n_{e}$ (green, solid) and $n_{i}$ (green, dashed) from HRTS, $T_{i}$ (blue) and $\Omega_{\Phi}$ (magenta) from the core $(\checkmark)$ and edge $(\bullet)$ CXRS systems with fitted input profiles (lines), where the errorbars represent $\sigma_{\text {data }}$ and raw data (points); radially integrated powers of components of the power balances of the ions (b) and the electrons (c), where the TRANSP profiles are averaged over the sustained ELMy H-mode phase $(8.5 \leq t \leq 10.0 \mathrm{~s})$ and the measurement data are shown at $9.5 \mathrm{~s}$. The various terms in the power balances are explained in $\S$ Appendix B.

transport model [65], which takes the neutral fuelling rate $\Gamma_{D_{2}}$ from a mid-plane measurement of the $\mathrm{D}_{\alpha}$ intensity, assuming $\Gamma_{D_{2}}=4 \pi(S / X B) I_{\mathrm{D}_{\alpha}}$ and the value $S / X B=10$ for the number of ionisations per photon. In the ETB region $Q_{c x, \text { net }}$ becomes large $\left(\lesssim 0.5 \mathrm{MW} / \mathrm{m}^{3}\right)$ because the CX neutrals are able to escape from the edge of the plasma. Inboard of the pedestal top $\left(\rho_{N} \lesssim 0.96\right)$, the integrated, net charge-exchange power $P_{C X}$ is small and negative $(\sim-0.05 \mathrm{MW})$, i.e. absorbsion of CX neutrals from the SOL/pedestal region, where the neutral density is high, deposits net power deeper into the plasma. Hence, the ion thermal transport power $P_{\text {tran }, i}$ reaching the pedestal top is $\sim 12 \mathrm{MW}$.

On average about a third of the total power lost through the electron channel $P_{\text {loss,e }}$ is due to radiation, $P_{R a d} \sim 7 \mathrm{MW}$, leaving the electron transport loss crossing the top of the pedestal, $P_{\text {tran }, e} \sim 13 \mathrm{MW}$. The fractions of the total transport loss power $P_{\text {tran }} \sim 25 \mathrm{MW}$ carried by the ions and electrons are hence $P_{\text {tran }, i} / P_{\text {tran }} \sim 0.48$ and $P_{\text {tran,e }} / P_{\text {tran }} \sim 0.52$, i.e. on average $\sim 1.1$ times more power is conducted to the pedestal top through the electrons than through the ion channel.

As the results shown in Fig. 13 are time-averaged over many ELM cycles, the total transport loss power $P_{\text {tran }} \sim 25 \mathrm{MW}$ includes the ELM energy losses and so should be compared with the sum of the inter-ELM loss power $P_{S e p}^{i E L M}$ and the time averaged ELM loss power $\left\langle P_{E L M}\right\rangle=f_{E L M} \times \Delta W_{E L M}$. Doing this we find it to be consistent with the values of $P_{S e p}^{i E L M}+\left\langle P_{E L M}\right\rangle \approx 23 \pm 0.6 \mathrm{MW}$ given in Table 3 for this pulse.

The partition of the ELM energy loss between the electrons and ions is not known, however, if we assume that $\left\langle P_{E L M}\right\rangle \sim 11 \mathrm{MW}$ is equally split between the ions and electrons, then $5.5 \mathrm{MW}$ is lost from each channel. This would leave the net, inter-ELM transport loss powers into the pedestal top of $12-5.5=6.5 \mathrm{MW}$ carried the ion channel and $13-5.5=7.5 \mathrm{MW}$ in the electron channel, i.e. $\sim 1.2$ times more power is conducted across the pedestal through the electron channel than through the ions. This fraction lies within the range of values resulting from the non-linear gyrokinetic calculations of Ref. [11] (see Fig. 8), which predict that between $\sim 25 \%$ (dominant Be impurity) and $\sim 55 \%$ (dominant Ni impurity) 

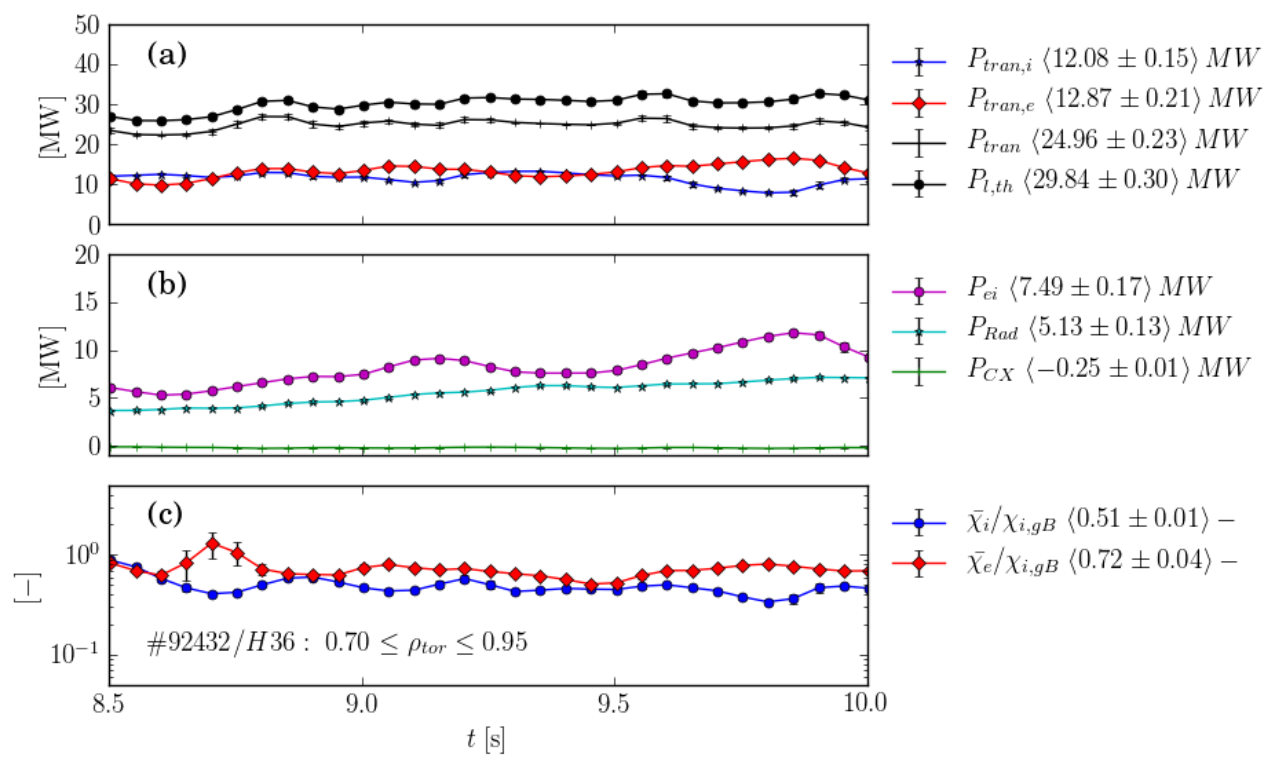

Figure 14: Results of interpretive, TRANSP power balance analysis of JET-ILW pulse \#92432 showing the evolution of: (a) the total loss power $P_{l, t h}(\bullet)$, the total transport loss power $P_{\text {Sep }}(+)$ and the transport losses in the ions $P_{\text {tran, }, i}(*)$ and electrons $P_{\text {tran,e }}(\$)$; (b) the ion-electron exchange power $P_{e i}(\bullet)$, the radiated power $P_{\text {Rad }}(*)$ and the charge-exchange power $P_{c x}(+)$ at $\rho_{\text {tor }}=0.9$; and (c) the gyro-Bohm normalised ion and electron thermal diffusivities $\chi_{i} / \chi_{i, g B}(\bullet)$ and $\chi_{e} / \chi_{i, g B}(\diamond)$ averaged over the mantle region $\left(0.7 \leq \rho_{\text {tor }} \leq 0.96\right)$.

of the power is conducted through the ion channel (including a $\sim 15 \%$ contribution from neoclassical transport), i.e. inter-ELM, electron/ion conducted power fractions $P_{\text {tran }, e} / P_{\text {tran }, i}$ in the range $\sim 0.8-3.0$. Accurate measurements of the ion dilution in the pedestal region would be required to pin down this fraction more accurately from the simulations.

The evolution of various powers from the TRANSP analysis just inside the pedestal top $\left(\rho_{N}=0.9\right)$ is shown in Fig. $14(\mathrm{a}, \mathrm{b})$, together with averaged values of these quantities over the specified time range. Wheras the total transport loss power $P_{\text {tran }} \sim 25 \mathrm{MW}$ is quite constant, the radiated power $P_{R a d}$ doubles during this phase, cooling the electrons in the mantle region and hence increasing the ion-electron exchange power $P_{e i}$ substantially. Consequently, the fraction of power conducted through the electrons to the pedestal top increases relative to that in the ion channel.

The electron and ion thermal diffusivities normalised to the gyro-Bohm level $\chi_{i} / \chi_{i, g B}$ and $\chi_{e} / \chi_{i, g B}$, averaged over the mantle region are shown in Fig. $14(\mathrm{c})$. As we are discussing core heat transport, the gyro-Bohm ion heat diffusivity is defined here as: $\chi_{i, g B}=v_{t h, i} \rho_{i}^{2} / a$, where $a$ is the minor radius. Time-averaged values of these normalised thermal diffusivities over the period shown are: $\chi_{i} / \chi_{i, g B} \sim 0.51$ and $\chi_{e} / \chi_{i, g B} \sim 0.72$, which is consistent with somewhat stronger turbulent electron than ion heat transport in the mantle region.

Time averaged profiles over the outer part of the plasma of the normalised temperature gradients $R / L_{T_{i}}$ and $R / L_{T_{e}}$ and the associated gyro-Bohm heat fluxes, the normalised $E \times B$ shearing rate $\gamma_{E} /\left(c_{s} / a\right)$ and thermal diffusivities are shown in Fig. 15 for this JET-ILW pulse and from a similar TRANSP run for the JET-C pulse \#78677 with matched $p_{e, p e d}$. For the JET-ILW pulse, over the mantle region $\chi_{e}$ exceeds $\chi_{i}$ by up to a factor $\lesssim 2$, except close to the 

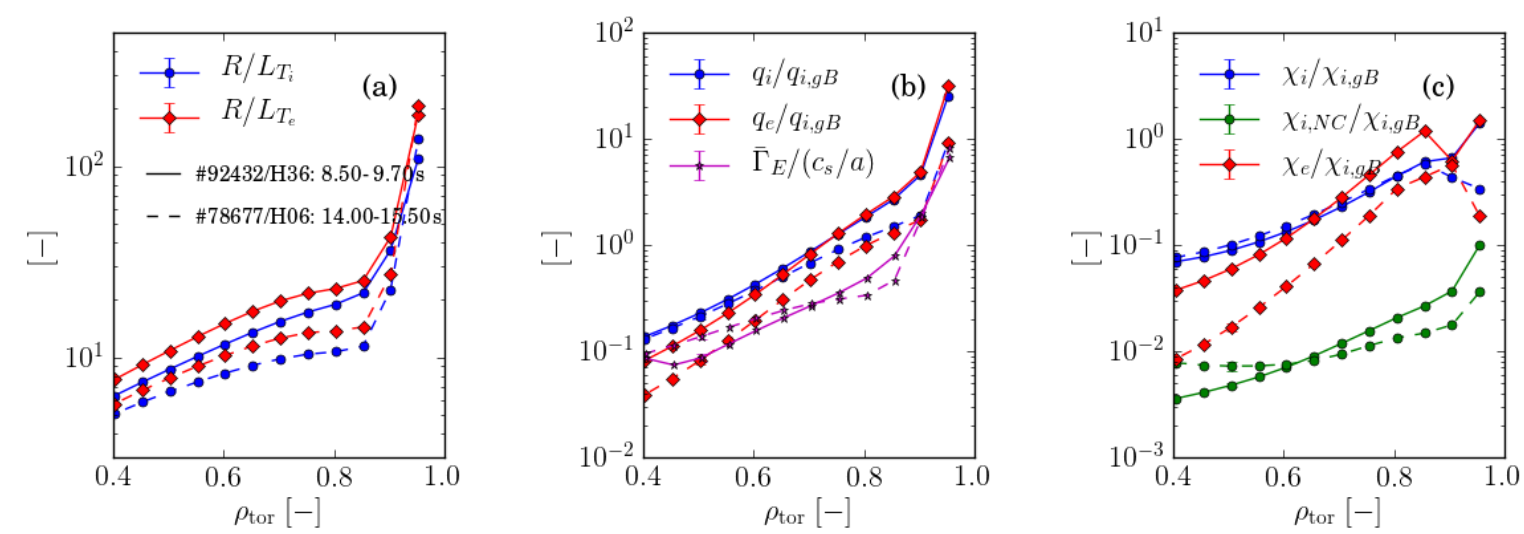

Figure 15: Results of interpretive, TRANSP power balance analysis of JET-ILW pulse \#92432 (solid) and JET-C pulse \#78677 (dashed) showing time-averaged profiles over the ELMy H-mode phases of: (a) the normalised temperature gradients $R / L_{T_{e}}(\diamond)$ and $R / L_{T_{i}}(\bullet)$; (b) the gyro-Bohm normalised electron and ion heat fluxes $q_{e} / q_{i, g B}(\diamond)$ and $q_{i} / q_{i, g B}(\bullet)$ and the normalised $E \times B$ shearing rate $\gamma_{E} /\left(c_{s} / a\right)(\star)$; and $(c)$ the gyro-Bohm normalised electron and ion thermal diffusivities $\chi_{e} / \chi_{i, g B}(\diamond)$ and $\chi_{i} / \chi_{i, g B}(\bullet)$.

pedestal top where $\chi_{e}$ approaches $\chi_{i}$. In the outermost region $\left(\rho_{N} \gtrsim 0.9\right)$ the $E \times B$ shearing rate becomes large enough that it might influence the level of ion-scale turbulence, i.e. when $\gamma_{E} /\left(c_{s} / a\right)>1$.

In contrast, we see that for the JET-C pulse \#78677, over most of the core plasma $\chi_{i} \gtrsim \chi_{e}$ (except near the pedestal top where they are equal), which is consistent with ion scale turbulence dominating the core heat transport. For completeness, the time-averaged kinetic profiles and results of interpretive power balance calculations for this JET-C pulse are presented in $\S$ Appendix $\mathrm{D}$, where we see that in the core the conducted power in the ion channel also exceeds that in the electron channel, i.e. $P_{\text {tran }, i} \gtrsim P_{\text {tran }, e}$ for $\rho_{\text {tor }} \leq 0.8$.

Note that an upgrade to the NBI systems between JET-C and JET-ILW operations altered both the fractions of total heating to the ions and electrons and their power deposition profiles [64]. As a consequence, in spite of the ICRH heating in the plasma core, the total heating power to the ions relative to that to the electrons $P_{\text {heat }, i} / P_{\text {heat, }}$ was less in the JETILW pulse \#92432 ( 1.14) compared to that in the JET-C pulse \#78677 ( 1.56). The power deposition profiles were also broader in the JET-ILW pulse, particuarly of the electron heating, which peaks in the outer regions of the plasma $\left(\rho_{t o r} \sim 0.8\right)$ where the $T_{e}$ is lower.

By cooling the electrons, the effect of the strong mantle radiation in the JET-ILW pulse is to increase the collisional exchange power from the ions and thereby the total power lost through the electron channel relative to that through the ions. The higher electron heat flux relative to that in the ion channel is consistent with the high values of $\eta_{e}$ prevalent in the mantle region, which can drive ETG or MTM turbulence. Indeed, linear, electron scale gyro-kinetic calculations indicate that electron scale modes dominate at the pedestal top [7].

Here, we are interested in the heat fluxes at the pedestal top, so are not concerned with the detailed power balance within the narrow ETB region $\left(\Delta R_{\text {ped }} \sim 2-4 \mathrm{~cm}\right)$, for which the spatial resolution $(\Delta R \sim 0.5 \mathrm{~cm})$ and validity of some assumptions of the TRANSP calculations are questionable. At the edge of the plasma, the poloidal ion gyro-radius $\rho_{i, \theta}=(q / \epsilon) \rho_{i} \sim 3 \mathrm{~cm}$ is 
of the order of the pedestal width $\dagger$.

Because $T_{i}>T_{e}$ within the ETB and SOL, the values of the ion-electron exchange power $Q_{i e}$ calculated using the local temperatures are large. The exchange power $Q_{i e}$ should, however, be calculated using spatially averaged quantities over the radial width of the ion banana orbits and not locally as assumed in TRANSP. In spite of these caveats, within the ETB the calculated ion-electron exchange and charge-exchange power densities $Q_{i e}$ and $Q_{c x, n e t}$ are both $\lesssim 0.5 \mathrm{MW} / \mathrm{m}^{3}$, almost halving the residual ion heat flux crossing the separatrix.

An approximate calculation of the radiative cooling of the electrons within the ETB can be made using the flux-surface averaged emissivity from the bolometer reconstructions of $\left\langle\epsilon_{m}\right\rangle \sim 0.2 \mathrm{MW} / \mathrm{m}^{3}$ (see Fig. $10(\mathrm{c})$ ) and an estimate of the volume of the ETB of $V_{E T B} \sim 5 \mathrm{~m}^{3}$, i.e. $P_{\text {Rad }}^{E T B}=\left\langle\epsilon_{m}\right\rangle V_{E T B} \sim 1 \mathrm{MW}$. Hence, only $\sim 4 \%$ of the total transport loss power $P_{S e p} \sim 25 \mathrm{MW}$ or $\sim 10 \%$ of the electron transport loss power $P_{\text {tran }, e} \sim \mathcal{O}(10) \mathrm{MW}$ is directly radiated from within the ETB. Hence, such direct cooling by impurity radiation (primarily from $\mathrm{W}$ ) from within the ETB region cannot explain the low temperature of the JET-ILW pedestals.

\section{Summary \& discussion}

The main results from each of the sections above are summarised and discussed below: $\S 2$ Determining ELM and inter-ELM loss powers: The inter-ELM loss power $P_{S e p}^{i E L M}$ can be estimated by averaging, over many ELM cycles, the instantaneous loss power $P_{S e p}$, calculated from power balance of the confined plasma. For this, we used the MHD stored energy $W_{M H D}$ from fast EFIT ++ equilibrium reconstructions as a proxy for the plasma kinetic energy $W_{t h}$. ELM energy losses $\Delta W_{E L M}^{\text {mag }}$ calculated from $W_{M H D}$ are generally not as reliable for JET-ILW pulses as was previously the case for JET-C. By comparing these losses with those evaluated from kinetic profile measurements $\Delta W_{E L M}^{k i n}$, we have shown that, for ELM frequencies $f_{E L M} \lesssim 40 \mathrm{~Hz}$, the ELM losses $\Delta W_{E L M}$ can be estimated to an accuracy of $\sim \pm 20 \%$. These losses can then be used to calculate the time-averaged, ELM loss power $\left\langle P_{E L M}\right\rangle \sim \Delta W_{E L M} \times f_{E L M}$. Within this limit on the ELM frequency, we have confirmed that there is good agreement between $\left\langle P_{E L M}\right\rangle$ and the average rate of change of stored energy $\left\langle d W_{M H D} / d t\right\rangle$ between ELMs, which must be the case in a steady H-mode phase, hence confirming our analysis to be internally consistent.

In $\S 3$, results of application of this analysis to two different sets of pulses were presented:

$\S 3.1$ 1.4 MA H-mode power scan in JET-ILW with low gas fuelling: Power balance analysis of this series of pulses reveals that the loss power fractions remain approximately constant over a factor three range of heating power $(4.6-16 \mathrm{MW})$, with radiation and the time-averaged ELM loss power each accounting for about 20-30\% and inter-ELM pedestal heat transport about $40-50 \%$ of the total losses. The pedestal temperature $T_{e, p e d}$ approximately doubles with this increase in power, while the pedestal density $n_{e, p e d}$ decreases by about $25 \%$, with concomitant changes to their radial gradients. The pedestal pressure $p_{e, p e d}$ (and pressure gradient) increases somewhat less than the temperature (and its gradient) due to the decreasing density. The ELM frequency also increases with increasing power, as expected for type-I ELMs. However, because $T_{e, p e d}$ and $p_{e, p e d}$ increase faster at higher power, they still reach higher pre-ELM values at the end of the ELM cycle. At the highest power, the pre-ELM $p_{e, p e d} \sim 4 \mathrm{kPa}$ is almost double that at the lowest power $(\sim 2.5 \mathrm{kPa})$. The parameter $\eta_{e}$ also increases with power, saturating

$\dagger$ The presence of a strongly sheared radial E-field within the ETB can reduce the width of the ion orbits $\Delta R_{b} \sim \rho_{i, \theta}-$ an effect referred to as 'orbit squeezing' (see Ref. [66]) 
at mean values over the ETB of $\left\langle\eta_{e}\right\rangle_{\text {ped }} \sim 2$, hence, ETG and/or ITG turbulent heat transport (assuming $\eta_{i}$ also increases with power) might be expected to be stronger at higher power.

Analysis of the dependence of the mean pedestal temperature gradient $\left\langle d T_{e} / d R\right\rangle_{\text {ped }}$ on the inter-ELM loss power $P_{S e p}^{i E L M}$ over the power scan reveals an approximately linear dependence, which implies a constant, effective thermal conductivity $\left\langle\chi_{e f f}\right\rangle \sim 0.6 \mathrm{~m}^{2} / \mathrm{s}$ across the pedestal. Hence, in terms of the integral response of the pedestal temperature to the increased heat flux, the pedestal heat transport is not particularly stiff. The issue of stiffness of the pedestal heat transport is examined in greater detail in $§ 3.1 .3$.

The ion neo-classical heat conduction across the pedestal is expected to be more significant in low plasma current $I_{p}$ pulses with low toroidal field $B_{\phi}\left(\chi_{i, N C} \propto 1 / B_{\phi}^{2}\right)$ and also at low heating power when the pedestal is more collisional. Calculation of neo-classical heat flux $q_{i, N C}$ across the pedestal in these $1.4 \mathrm{MA} / 1.7 \mathrm{~T}$ pulses using an appropriate expression for $\chi_{i, N C}$, valid for arbritrary $\nu_{i, p e d}^{\star}$, reveals that at the lower heating powers, a substantial fraction of the total heat flux $q_{t o t}$ is due to collisional, ion neo-classical transport. Although the $q_{i, N C}$ increases with the heating power $P_{a b s}$, the relative magnitude of the total heat flux $q_{t o t}$ to the neo-classical component $q_{t o t} / q_{i, N C}$ increases as $\nu_{i, p e d}^{\star}$ decreases with the heating power $P_{a b s}$.

The ratio of the total pedestal heat flux normalised to the ion gyro-Bohm heat flux $q_{t o t} / q_{i, g B}$, which is a measure of the intensity of the turbulence, is found to increase with the ratio $q_{t o t} / q_{i, N C}$, which is consistent with an increase in the intensity of the turbulent component. The total inter-ELM loss power across the pedestal $P_{S e p}^{i E L M}$ also increases with the mean normalised pedestal temperature gradient $\left\langle\eta_{e}\right\rangle_{\text {ped }} \sim 1-2$, which modestly exceeds the predicted threshold for the 'slab' branch of ETG turbulence $\eta_{e, c r}^{S} \sim 0.8$. Note that it has also recently been pointed out that slab ETG turbulence plays a very important role in the heat transport in JET-ILW pedestals [58].

The trend for the turbulent heat flux $q_{t u r b}$ to increase with $\left\langle\eta_{e}\right\rangle_{p e d}$ implies that the pedestal heat transport is not very stiff, i.e. the $R / L_{T_{e}}$ is not strongly clamped at a critical gradient, e.g. the predicted value of $\left(R / L_{T_{e}, c r}\right)^{S}=0.8 * R / L_{n_{e}}$ for 'slab' ETG turbulence. A simple model assuming infinitely stiff pedestal heat transport, i.e. that $\eta_{e}$ is strongly clamped to $\eta_{e, c r}$ in the pedestal region, yields the simple prediction: $T_{e, p e d}^{\star}=T_{e, s e p}\left\langle n_{e, p e d} / n_{e, s e p}\right\rangle^{\eta_{e, c r}}$. Under conditions where the turbulent heat flux dominates, $T_{e, p e d}$ would be largely independent of the heat flux $q_{t o t}$ across the pedestal provided the density profile was unchanged. However, the observation that $\left\langle\eta_{e}\right\rangle_{p e d}$ increases with $q_{t o t}$, i.e. that the pedestal heat transport is not infinitely stiff, does allow $T_{e, p e d}$ to increase as the heating power $P_{a b s}$ is increased, even though there is little change in the density profile.

$\S 3.2$ Comparison of high-power, 3.0 MA 'baseline' pulses in JET-C and JET-ILW: In highpower, 3.0 MA baseline scenario JET-ILW pulses substantially more heating power is required to achieve the same normalised confinement $\left(H_{98, y} \sim 1\right)$ and pedestal pressure $p_{e, p e d}$ as in comparable JET-C pulses. The fact that the resulting pedestal temperature is much lower in the JET-ILW pulses implies an increase in the virulence of the inter-ELM pedestal heat transport. Analysis of the loss power components for these pulses reveals that, while the ELM loss power fraction is about half in the JET-ILW pulses (due to the smaller ELM energy losses), both the fractions of radiated power and the pedestal heat transport are about double those in the JET-C pulses.

Average values of the inter-ELM loss power $P_{\text {Sep }}^{i E L M}$ are quoted Table 3 for comparison with numerical simulations, i.e. 11-13 MW for the JET-ILW pulses and 6-7 MW for the JET$\mathrm{C}$ pulses. In the case of the JET-ILW pulses, this level of pedestal heat transport compares well with the results of the non-linear gyro-kinetic simulations of Ref. [11]. Typically, higher 
mean values of $\eta_{e}$ prevail in the pedestal region of these JET-ILW pulses than in the JET-C pulses, which is consistent with stronger temperature gradient driven turbulent transport in the JET-ILW pedestals.

$\S 4$ Radiation and power balance in high-power, 3 MA 'baseline' scenario JET-ILW pulses $\S 4.1$ Evidence for $W$ as the dominant radiator: Our analysis of the poloidal distribution of the total radiation reveals that the $30-40 \%$ radiated power fraction in the high-power, $3 \mathrm{MA}$ JET-ILW baseline scenario pulses is dominated by that from $\mathrm{W}$ impurities. This conclusion is supported by results of an integrated analysis of SXR, bolometer and line-of-sight integrated $Z_{\text {eff }}$ data [46], which shows that a mean concentration of $\sim 2 \times 10^{-4}$ of $\mathrm{W}$ radiates $\sim 96 \%$ of the total radiation, while the $\mathrm{Ni}$ impurities (at half of the $\mathrm{W}$ concentration) contribute only $\sim 2 \%$ of the radiation. Most of the $\sim 17 \%$ ion dilution is due to Be impurities with an assumed constant concentration of $\sim 3 \%$.

\section{$\S 4.2$ Response of $W$ and heat transport to edge gradients}

$W$ transport: The temperature and density gradients in the pedestal and mantle regions are typically aligned such that strong neo-classical convection is directed $\left(\zeta_{N C}<0\right)$ to drive impurities in the SOL inwards across the ETB to the pedestal top. During the sustained ELMy H-mode phase, outward convection $\left(\zeta_{N C}>0\right)$ in the mantle region helps to localise these impurities there and hinders their accumulation in the plasma core. While concentrated in the mantle region, the impurites can be effectively flushed from the plasma by the ELMs.

Heat transport: In this section we showed that the threshold behaviour of ETG driven heat transport is broadly consistent with the temperature and density profile behaviour in pedestal and mantle regions of these JET-C and JET-ILW pulses:

Pedestal: In both the JET-ILW and JET-C 3 MA pulses, the mean normalised temperature gradient parameter $\left\langle\eta_{e}\right\rangle_{\text {ped }} \sim 1-2$ across the pedestal, is broadly consistent with a predicted, constant threshold for the slab branch of ETG turbulent transport, i.e. $\eta_{e, c r}^{S} \sim 0.8$ [58]. Using average values of the pedestal to separatrix density ratio $\left\langle n_{e, p e d} / n_{e, s e p}\right\rangle$ and assuming $T_{e, p e d} \sim 100 \mathrm{eV}$ and a value for $\eta_{e, c r}=2$, a simple model for stiff pedestal heat transport roughly predicts the mean $\left\langle T_{e, p e d}\right\rangle$ in both the JET-C and JET-ILW pulses. Hence, in terms of this simple model, the lower $T_{e, p e d}$ in the JET-ILW pulses is consistent with the relatively higher relative separatrix density $\left\langle n_{e, s e p} / n_{e, p e d}\right\rangle$ resulting from the strong gas puffing.

Mantle: In the mantle region, the weaker density gradient in the JET-ILW compared to that in the JET-C pulses results in different evolution of the parameter $\left\langle\eta_{e}\right\rangle_{\text {man }}$, which is perhaps explicable in terms of the predicted critical $\eta_{e, c r}$ for ETG turbulence. In the JET-C mantle, the observed constant $\left\langle\eta_{e}\right\rangle_{\text {man }} \sim 2$ is broadly consistent with the constant critical $\eta_{e, c r}^{S} \sim 0.8$ for slab ETG turbulence. However, in the JET-ILW mantle $\left\langle R / L_{n_{e}}\right\rangle_{\text {man }}$ is weaker, so the a constant threshold $\left(R / L_{T_{e}, c r}\right)^{T}$ for the toroidal branch of ETG turbulence is more applicable. The observed, quite constant value of $\left\langle R / L_{T_{e}}\right\rangle_{\text {man }} \sim 15-20$ is well above the predicted threshold $\left(R / L_{T_{e}, c r}\right)^{T} \sim 4$ [43], so we might expect strong ETG driven turbulent heat transport in this region.

$\S 4.3$ Interpretive transport analysis: Interpretive transport analysis of the high-power JET-ILW pulse \#92432, including the measured radiation profile, reveals that collisional exchange from the ions transfers almost as much power to the electrons as from the external heating systems and nearly equilibrates the ion and electron temperatures in the mantle region. Consequently, the heat flux in the electron channel into the pedestal region is about $20 \%$ higher than that in the ion channel. The total conducted loss power, which includes ELM losses, is consistent with our earlier power balance analysis. In the mantle region, where the normalised temperature gradient $\left\langle\eta_{e}\right\rangle_{\text {man }}$ exceeds the predicted threshold for the toroidal branch of ETG turbulence, the resulting gyro-Bohm thermal diffusivity of the electrons $\chi_{e} / \chi_{i, g B} \sim 0.72$ is higher than 
that of the ions $\chi_{i} / \chi_{i, g B} \sim 0.51$, consistent with the presence of strong turbulent electron heat transport in this region.

Estimates of the direct radiative cooling of the pedestal shows that this can account for only $\sim 4 \%$ of the total loss power through the pedestal or $\sim 10 \%$ of the conducted power in the electron channel. Hence, such direct cooling by $\mathrm{W}$ radiation is not the cause of the low pedestal temperatures prevelant in JET-ILW pulses. Instead, this is likely to be due to the higher levels of turbulent heat transport across the pedestal. In a simple model assuming stiff turbulent heat transport, clamping $\eta_{e}$ to a threshold value across the pedestal, the enhanced separatrix density at high levels of gas fuelling results in a lower pedestal temperature. The strong $\mathrm{W}$ radiation from the confined plasma does, however, cause a larger fraction of the conducted loss power to be carried by the electrons than that by the ions in these JET-ILW pulses compared to that in the JET-C pulses.

\section{Conclusions}

In JET-ILW pulses, relatively strong gas puffing is required to achieve steady-state ELMy Hmode operation at high heating power, both to reduce the sputtered $\mathrm{W}$ source and to maintain the rate of flushing of the $\mathrm{W}$ impurities by ELMs from the confined plasma. A deliterious effect of this strong gas puffing is to increase separatrix density relative to that at the pedestal top. This reduces the pedestal density gradient, which increases the drive for temperature gradient driven micro-turbulence and thereby reduces the achievable pedestal temperature. Our calculations of the inter-ELM pedestal loss power show that the heat flux required to maintain a similar pedestal pressure is considerably higher in JET-ILW pulses (even taking into account the increased heating power) than in similar JET-C pulses - a direct result of more virulent turbulent heat transport across the pedestal. The calculated inter-ELM loss powers are consistent with results of non-linear, gyro-kinetic simulations of the same JET-C and JET-ILW pulses [11].

Previous results comparing the confinement of JET-C and JET-ILW pulses with matched engineering parameters [48] show, however, that $T_{e, p e d}$ was significantly lower in the JET-ILW baseline-scenario pulses than in the JET-C pulses, in spite of these having been run with the same rate of gas fuelling. A possible explanation for this might be related to the higher fuel dilution (lower $n_{i} / n_{e}$ ) by $\mathrm{C}$ in the JET-C pulses, which is known to reduce the growth rates of ion-scale turbulence. A beneficial effect of the reduced fuel dilution in JET-ILW with the $\mathrm{Be} / \mathrm{W}$ wall is, however, to increase the ion pressure in the core plasma relative to that in comparable JET-C pulses. Hence, the overall normalised pressure $\beta_{N}$ is somewhat higher in JET-ILW pulses than in JET-C pulses with comparable $p_{e, p e d}$ and normalised confinement factor $H_{98, y}$.

The effect of the strong $\mathrm{W}$ radiation from the core plasma (radiated power fraction $\mathcal{F}_{\text {Rad }} \sim 20-40 \%$ ) in the high-power JET-ILW pulses is to cool the electrons, particularly in the outer mantle region, and hence increase the collisional heating of the electrons by the ions. This increases the fraction of the total power conducted through the electron channel relative to that through the ions both across the plasma core to the pedestal top and ultimately across the pedestal. Turbulent electron heat transport is hence relatively more significant in the JET-ILW pulses than was the case in JET-C pulses, in which the heat conduction through the ion channel dominated heat transport in the core plasma.

During the sustained, ELMy H-mode phase of the high-power ITER-baseline pulses in JET-ILW, the weak density gradient in the mantle results in outward neo-classical convection of the $\mathrm{W}$ impurities, helping to prevent their accumulation in the plasma core. If the ELM 
frequency is insufficient to flush sufficient $\mathrm{W}$ from the confined plasma, the resulting build-up of the $\mathrm{W}$ content and hence $\mathcal{F}_{\text {Rad }}$ causes the loss power across the pedestal $P_{\text {Sep }}^{i E L M}$ to fall below the thereshold require to sustain the $\mathrm{H}$-mode. The resulting $\mathrm{L} \rightarrow \mathrm{H}$-mode transition rapidly modifies the density and temperature profiles, leading to a sudden inward convection of the $\mathrm{W}$ impurities from the mantle region into the plasma core, which leads to the termination of the high-performance phase of the pulse $[57,69]$.

While the $\mathrm{W}$ content can be controlled by increasing the gas fuelling rate and hence the ELM frequency, as discussed above, this reduces $T_{e, p e d}$ and hence the overall confinement. An alternative means of limiting the buildup of the $\mathrm{W}$ impurity content is to maintain the ELM frequency using a succession of deuterium 'ELM-pacing' pellets. Operation with such pellets has extended the period of ELMy H-mode operation in such high-power (33 MW) 3 MA JETILW baseline scenario pulses with a reduced $(\sim 30 \%)$ rate of gas puffing and lower $(\sim 70 \%)$ overall particle throughput compared to similar pulses fuelled only by gas puffing [68]. The pacing pellets result in more frequent, smaller ELMs and other beneficial effects, including higher core rotation and ion temperature and hence improved overall energy confinement [69].

\section{Acknowledgements}

This work has been carried out within the framework of the EUROfusion Consortium and has received funding from the EURATOM Research and Training Programme 2014-2018 and 2019-2020 under grant agreement No. 633053 and from RCUK Energy Programme [grant No. $\mathrm{EP} / \mathrm{T} 012250 / 1]$. The views and opinions expressed herein do not necessarily reflect those of the European Commission.

\section{References}

[1] Giroud C et al 2013 Nucl. Fusion 53113025

[2] Beurskens M N A et al 2013 Plasma Phys. and Contr. Fusion 55124043

[3] Leyland M J et al 2015 Nucl. Fusion 55013019

[4] Maggi C F et al 2015 Nucl. Fusion 55113031

[5] Maggi C F et al 2017 Nucl. Fusion 5711612

[6] Stefanikova E et al 2018 Nucl. Fusion 58056010

[7] Frassinetti L et al 2018 Nucl. Fusion 59076038

[8] Hatch D R et al 2016 Nucl. Fusion 56104003

[9] Hatch D R et al 2017 Nucl. Fusion 57036020

[10] Kotchenreuther M et al 2017 Nucl. Fusion 57064001

[11] Hatch D R et al 2019 Nucl. Fusion. 59086056

[12] Dickinson D et al 2012 Phys. Rev. Lett. 108135002

[13] Appel L et al 2006 Proc $33^{\text {rd }}$ Conf. on Contr. Fusion and Plasma Phys., Rome, EPS Geneva (2006) ECA Vol. 30I P2.184

[14] Shafranov V D 1963 J. Nucl. Energy, Part C Plasma Phys. 5251

[15] Shafranov V D 1971 Plasma Physics 13757

[16] Zakharov L E and Shafranov V D 1973 Sov. Phys. Tech. Phys. (Engl. Transl.) 182 151-156

[17] Lao L et al 1985 Nucl. Fusion 251611

[18] Challis C et al 2015 Nucl. Fusion 55053031

[19] Angioni C et al 2014 Plasma Phys. and Contr. Fusion 56124001

[20] Challis C D et al 1989 Nucl. Fusion 29563

[21] Huber A et al 2007 Proc. 24 $4^{\text {th }}$ Symp. on Fusion Technology SOFT-24, Fusion Eng. and Design 82 5-14 1327-1334 
[22] Field A R et al 2017 Plas. Phys. Control. Fusion 59095003

[23] Ingesson L C et al 1998 Nucl. Fus. 38111675

[24] Pasqualotto R et al 2004 Rev. Sci. Inst. $75103891-3893$

[25] Frassinetti L et al 2012 Rev. Sci. Inst. 83013506

[26] Frassinetti L et al 2015 Nucl. Fusion 56023007

[27] Dickinson D et al 2011 Plas. Phys. Control. Fusion 5311115010

[28] Saarelma S et al 2015 Phys. Plasmas 22056115

[29] Simpson J et al 2019 Nucl. Mat. \& Energy 20100599

[30] Connor J W \& Wilson H R 1994 Plas. Phys. Control. Fusion 365719

[31] Garbet X et al 2004 Plas. Phys. Control. Fusion 46 B557

[32] Bendat J S and Piersol A G 2010 Random Data, Analysis and measurement procedures, Wiley, New Jersey, $4^{\text {th }}$ Edition, ISBN 978-0-470-24877-5

[33] Wesson J 2004 Tokamaks, 3rd Ed., Clarendon Press - Oxford, ISBN 0-19-8509227

[34] Chang C S and Hinton F L 1986 Phys. Fluids 293314

[35] ITER Physics Expert Group on Confinement \& Transport 1999 ITER Physics Basis, Ch. 2, Nucl. Fusion 392175

[36] Houlberg W A 1997 Phys. Plasmas 43230

[37] Belli E A and Candy J 2008 Plas. Phys. Control. Fusion 50095010

[38] Hawryluk R 1979 An empirical approach to tokamak transport, Physics of plasmas close to thermonuclear conditions in Physics of Plasmas Close to Thermonuclear Conditions, ed. by B. Coppi, et al., (CEC, Brussels, 1980), Vol. 1, pp. 19-46.

[39] Haskey S R et al 2018 Plas. Phys. Control. Fusion 60105001

[40] Andrew Y et al 2006 Rev. Sci. Inst. 77 10E913

[41] Abel I G et al 2013 Rep. Prog. Phys. 76116201

[42] Chapman B et al 2020 'The impact of increased power and fuelling on the instability of JET-ILW pedestals' to be presented at $47^{\text {th }}$ Conf. on Plasma Phys., 22-26 June 2020, Sitges, Spain.

[43] Jenko F et al 2001 Phys. Plasmas 894096

[44] Romanelli F et al 1989 Phys. Fluids B 11018

[45] Hatch D P et al 2018 Plas. Phys. Control. Fusion 60084003

[46] Sertoli M et al 2018 Rev. Sci. Inst. 89113501

[47] Brezinsek S et al 2013 Nucl. Fus. 53083023

[48] Joffrin E et al 2014 Nucl. Fus. 54013011

[49] Challis C D et al 2004 Plas. Phys. Control. Fusion 46 B23-B40

[50] Perez C P et al 2003 Plasma Phys. and Contr. Fusion 4661

[51] Wenniger R P et al 2014 Nucl. Fusion 54114003

[52] Casson F et al 2014 Plas. Phys. Control. Fusion 57014031

[53] Wesson J A et al 1997 Nucl. Fusion 37578

[54] Henderson S S et al 2017 Plasma Phys. and Contr. Fusion 59055010

[55] Lux $\mathrm{H}$ et al 2014 ADAS Workshop 2014

[56] de la Luna E et al 2020 Exploring the physics of hot-ion H-mode with small ELMs and zero gas puffing in JET-ILW to be presented at the $28^{\text {th }}$ IAEA Fusion Energy Conf., Nice, France, $12-17$ October 2020.

[57] Field A R et al 2020 The impact of fuelling and $W$ radiation on the performance of high-power $H$-mode plasmas in JET-ILW to be presented at the $28^{\text {th }}$ IAEA Fusion Energy Conf., Nice, France, 12-17 October 2020.

[58] Parisi J et al Toroidal and slab ETG dominance in JET pedestals International Sherwood theory conference, Princeton Plasma Physics Laboratory, Princeton, NJ, 15-17th April 2019.

[59] Angioni C et al 2015 Phys. Plasmas 22055902

[60] Angioni C et al 2003 Phys. Rev. Lett. 90205003

[61] Weisen $\mathrm{H}$ et al 2005 Nucl. Fusion 45 L1

[62] Goldston R et al 1981 J. Comput. Phys. 43 61-78 
[63] Kim H T et al 2018 Nucl. Fusion 58036020

[64] Ćirić D et al 2007 Fusion Eng. Design 82610

[65] Tomar S et al 1981 J. Comput. Phys. 40104

[66] Shaing K C 1992 Phys. Fluids B 42547

[67] Grierson B A 2018 Fusion Science and Technology 74:1-2 101-115

[68] Garzotti L et al 2019 Nucl. Fusion 59076037

[69] Field A R et al 2019 Proc 46 ${ }^{\text {th }}$ Conf. on Contr. Fusion and Plasma Phys., Milan, EPS Geneva (2019), ECA Vol. 43C P5.1019

\section{Appendix A. Signal processing algorithm for estimating radiated power}

We can decompose the fast bolometer signal $P_{R a d}^{H o r}$ into low- and high-pass filtered components: $P_{\text {Rad }}^{H P}$ and $P_{\text {Rad }}^{L P}$, with a common cutoff frequency of $10 \mathrm{~Hz}$. By scaling the high-pass filtered signal $P_{R a d}^{H P}$ by the ratio of the more reliable $\left\langle P_{R a d}^{P l}\right\rangle$ signal from the tomographic reconstructions to the low-pass $P_{R a d}^{L P}$ signal and adding the scaled signal to $\left\langle P_{R a d}^{P l}\right\rangle$ we can construct a signal that retains the time response of the fast signal and also has the correct magnitude, i.e. $P_{\text {Rad }}^{P l}=\left\langle P_{\text {Rad }}^{P l}\right\rangle+\left(\left\langle P_{\text {Rad }}^{P l}\right\rangle / P_{\text {Rad }}^{L P}\right) \times P_{\text {Rad }}^{H P}$. Note that in this expression, each binary arithmetic operation implies interpolation of the signal on the RHS of the operator onto that on the LHS, i.e. of the signal with the faster timebase onto that with the slower timebase.

\section{Appendix B. Definition of terms in TRANSP power balance}

The equations governing the power, particle and momentum balances that underly the TRANSP calculations are explained in detail in Ref. [67]. The power balances are expressed in terms of power densities $Q$ and divergence of the conducted and convected heat fluxes $\nabla \cdot\left(q_{\text {cond }}+q_{\text {conv }}\right)$. For the ions, the power balance can be expressed in terms of power densities as:

$$
\dot{\mathcal{W}}_{i}=Q_{i, \text { heat }}+Q_{i e}-\nabla \cdot\left(q_{\text {cond }, i}+q_{\text {conv }, i}\right)-Q_{c x, n e t}+Q_{\text {rot }}
$$

, where $\dot{\mathcal{W}}_{i}=\frac{d}{d t}\left(\frac{3}{2} n_{i} T_{i}\right)$ is the rate of change of ion thermal energy density, $Q_{i, \text { heat }}$ is the total power heating the ions, $Q_{i e}$ is the exchange power density from the electrons to the ions, $Q_{c x, n e t}$ is the net charge-exchange loss power and $Q_{r o t}$ is the rotational heating. The electron-ion exchange power is given as: $Q_{i e}=\frac{3}{2} n_{e} \frac{\left(T_{e}-T_{i}\right)}{\tau_{i e}}$, where $\tau_{i e} \propto T_{e}^{3 / 2} / n_{e}$ and is hence larger in the outer regions where the $T_{e}$ is lower than in the core. The rotational heating $Q_{r o t}$ due to convection and friction is negligbly small compared to the other terms.

Each of the terms $Q_{X}$ in this equation can be volume integrated $P_{X}\left(\rho_{N}^{\prime}\right)=$ $\int_{0}^{\rho_{\text {tor }}^{\prime}} Q_{X}\left(d V / d \rho_{\text {tor }}\right) d \rho_{\text {tor }}$ to give the associated power deposited within or leaving the $\rho_{\text {tor }}^{\prime}$ flux surface. The rate of change of ion thermal energy $W_{i}$ within this flux surface can then be expressed in terms of equivalent powers as:

$$
\dot{W}_{i}=P_{\text {heat }, i}+P_{i e}-\left(P_{\text {cond }, i}+P_{\text {conv }, i}\right)-P_{c x}+P_{\text {rot }}
$$

For the electrons the power balance can be similarly expressed in terms of power densities or volume integrated quantities as:

$$
\begin{aligned}
& \dot{\mathcal{W}}_{e}=Q_{e, \text { heat }}+Q_{e i}-\nabla \cdot\left(q_{\text {cond }, e}+q_{\text {conv }, e}\right)-Q_{0, \text { ion }}-Q_{\text {Rad }} \\
& \dot{W}_{e}=P_{\text {heat }, e}+P_{e i}-\nabla \cdot\left(P_{\text {cond }, e}+P_{\text {conv }, e}\right)-P_{0, \text { ion }}-P_{\text {Rad }}
\end{aligned}
$$


, where $Q_{0, i o n}$ is the power required to ionise the neutrals and $Q_{R a d}$ is the total radiated power density, i.e. that available from the bolometer tomography as $\left\langle\epsilon_{m}\right\rangle$. Obviously, the ion-electron exchange power is balanced by that from the electrons to the ions, i.e. $Q_{e i}=-Q_{i e}$.

We can define two further powers for the ions: the loss power due to ion heat transport $P_{\text {tran }, i}=P_{\text {cond }, i}+P_{\text {conv }, i}$ and the total loss power from the ions $P_{\text {loss }, i}=P_{\text {tran }, i}+P_{c x}$. Similarly for the electrons: the power due to electron heat transport $P_{\text {tran }, e}=P_{\text {cond }, e}+P_{\text {conv }, e}$ and the total loss power from the electrons, including ionisation and radiation $P_{\text {loss }, e}=$ $P_{\text {tran }, e}+P_{0, i o n}+P_{\text {Rad }}$. The total transport loss power is $P_{\text {tran }}=P_{\text {tran }, e}+P_{\text {tran }, i}$.

\section{Appendix C. Integrated radiation analysis for JET-ILW pulse \#92432}
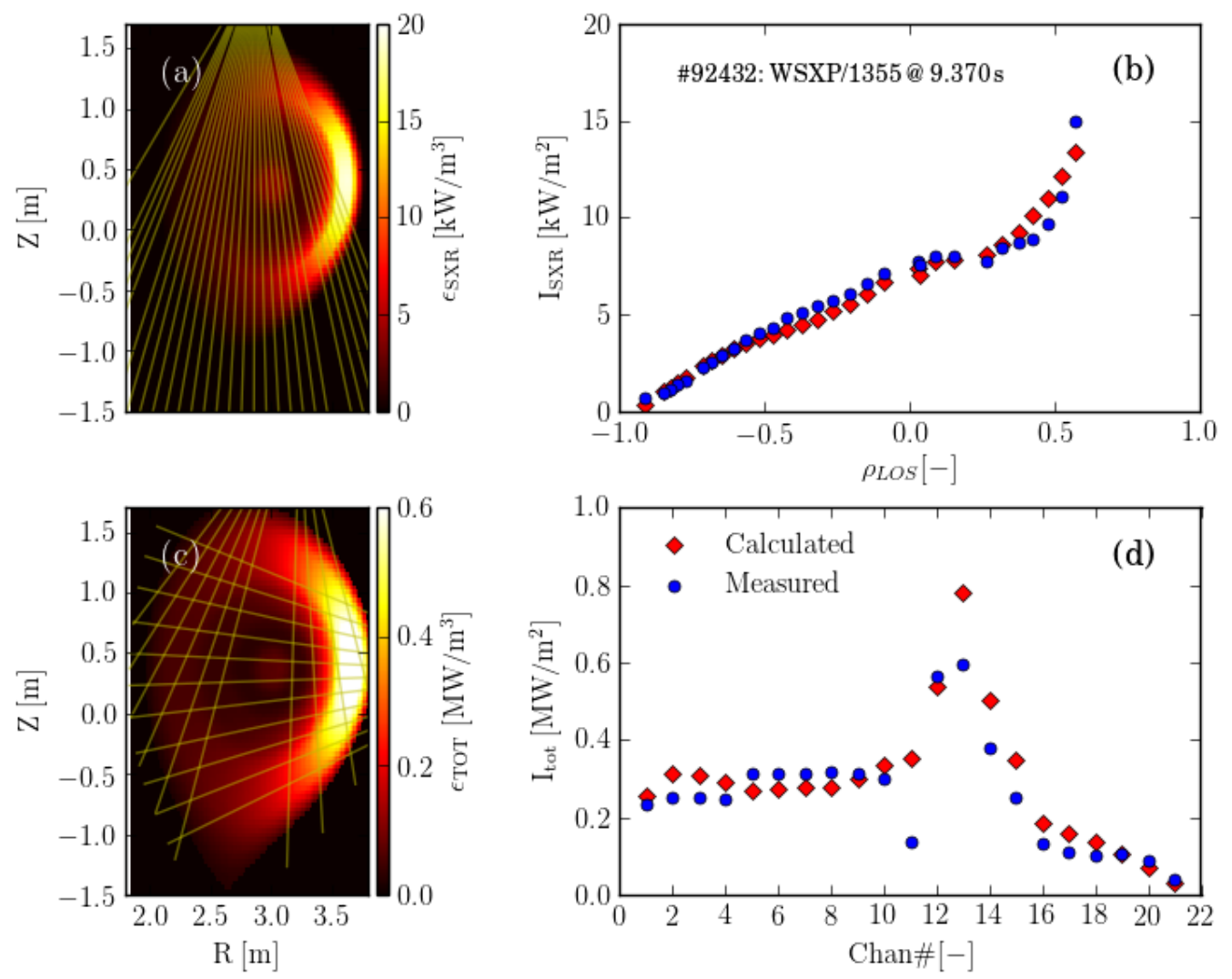

Figure C1: Results of the integrated analysis of soft x-ray (SXR), bolometric radiation and lineaveraged $Z_{\text {eff }}$ measurement data for JET-ILW pulse \#92432 at $9.37 \mathrm{~s}$ showing: distributions of (a) $S X R$ emissivity $\epsilon_{S X R}(R, Z)$; (b) total radiated emissivity $\epsilon_{\text {tot }}(R, Z)$; and a comparison of measured (•) and back-calculated ( $\$$ ) line-integrated intensities of: (c) SXR emission as a function of the tangency radii of the lines of sight $\rho_{L O S}$ in terms of $\rho_{\text {tor }}$ and (d) total radiation intensity $I_{\text {tot }}$ for each bolometer channel (\#1-11 horizontal, \#12-21 vertical). The diagnostic lines of sight are shown in $(a, c)$ in yellow.

\section{Appendix D. Interpretive transport analysis of JET-C pulse \#78677}

Kinetic profiles averaged over the steady, ELMy H-mode phase and results of interpretive power balance calculations for the JET-C pulse \#78677 are shown in Fig. D1. Note that, 
as for the JET-ILW pulse \#92432, the measurement data are mapped to flux coordinates using pressure constrained EFIT ++ equilibrium reconstructions with the additional, weakly weighted constraint to ensure $T_{e, s e p} \sim 100 \mathrm{eV}$.
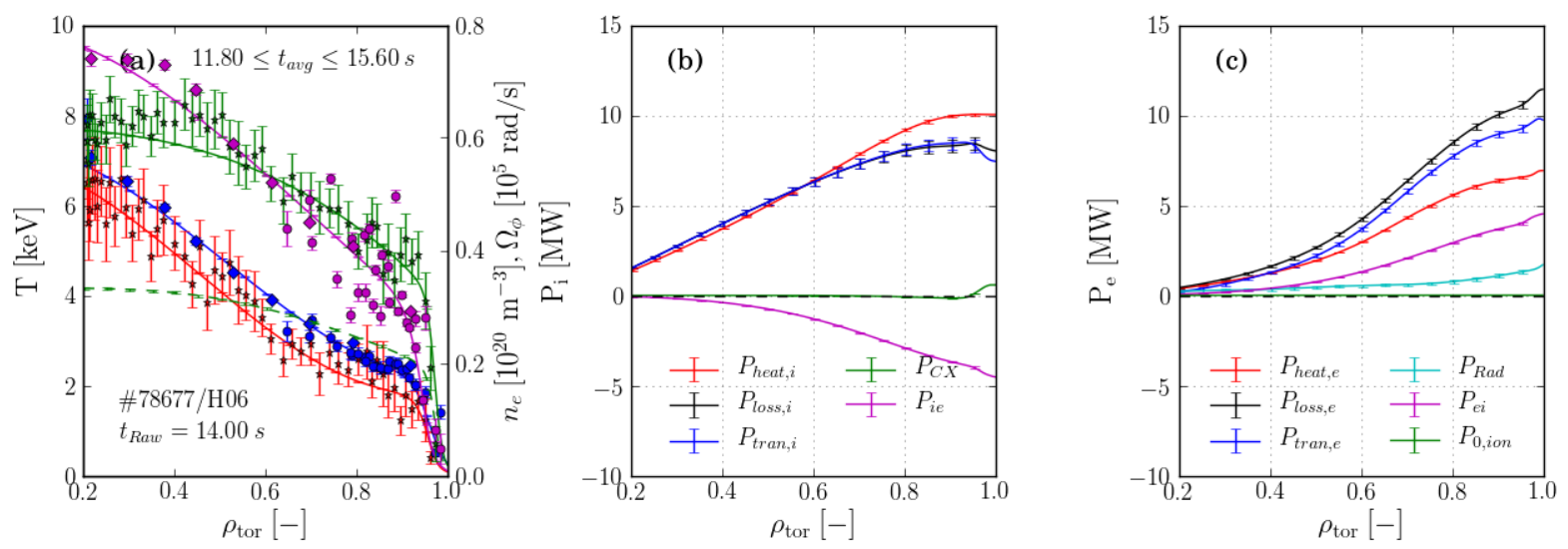

Figure D1: Results of interpretive, TRANSP power balance analysis of JET-C pulse \#78677, showing: (a) kinetic profiles $T_{e}$ (red), $n_{e}$ (green, solid) and $n_{i}$ (green, dashed) from HRTS, $T_{i}$ (blue) and $\Omega_{\Phi}$ (magenta) from the core $($ ) and edge $(\bullet)$ CXRS systems with fitted input profiles (lines), where the errorbars represent $\sigma_{\text {data }}$ and raw data (points); radially integrated powers of components of the power balances of the ions (b) and the electrons (c), where the TRANSP profiles are averaged over the sustaned ELMy H-mode phase $(11.8 \leq t \leq 15.6 \mathrm{~s})$ and the measurement data are shown at $14.0 \mathrm{~s}$. The various terms in the power balances are explained in $\S$ Appendix B. 